\title{
Advances in Photocatalytic Organic Synthetic Transformations in Water and Aqueous Media
}

\author{
Sebastián Barata-Vallejo,* Damian E. Yerien, and Al Postigo*
}

Cite This: https://doi.org/10.1021/acssuschemeng.1c03384

Read Online

ACCESS | Llll Metrics \& More | 回 Article Recommendations

ABSTRACT: Advances in visible light-photocatalyzed preparative synthetic organic transformations in water or aqueous media are studied. Both the convenience of visible light photocatalytic strategies to achieve synthetic organic transformations and the employment of water or aqueous mixtures make this approach a very resourceful methodology. Mechanistic aspects for such photocatalyzed processes are discussed. The effects of water in the stereoselectivity of some reactions and considerations regarding the improved enantioselectivity in chiral catalytic photoredox reactions are manifested with the use of water. The use of water as a result of a protocol improvement or environmentally friendlier methodology as compared to the same reaction performed in organic solvents are also considered. All these combined effects of water as a reaction medium or its very presence in photocatalytic reactions have an ultimate goal which is to diminish the environmental impact of synthetic and photocatalytic processes. This perspective highlights the progress and state of the art organic transformations realized by photocatalytic techniques in water or aqueous systems as well as water as a reactant. In this regard, aromatic substitution reactions, radical additions to carbon-carbon double bonds, photooxidation reactions, decarboxylation processes, and miscellaneous reactions are presented.

KEYWORDS: Photocatalysis in water, Enantioselective photocatalysis, Diastereoselective photocatalysis, Photocatalyzed arylations, Decarboxylative reactions, Photocatalyzed deoxygenations, Photocatalyzed photooxidations, Photocatalyzed cyclizations,

Photocatalyzed ATRA

\section{INTRODUCTION}

Visible light photoredox reactions have recently found many applications, occurring under mild conditions and not requiring radical chemical initiators nor stoichiometric amounts of chemical reductants or oxidants for redox neutral reactions. As an irradiation source, visible light reduces waste and curtails reaction expenses significantly. Photoredox catalysis is also tolerant of many functional groups present in organic scaffolds. ${ }^{1}$ On the other hand, water or water mixtures with organic solvents is another facet of green chemistry that has been endorsed by organic chemists and industry alike. In this sense, the association of light and water could offer new perspectives and increase the chemists' armamentarium for functionalization reactions triggered by visible light photoredox catalysis, as both water and visible light are safe, inexpensive, and clean. ${ }^{2,3}$

The majority of photocatalytic transformations conducted in water promote the removal of contaminants, either organic or organometallic. ${ }^{4}$ This is often done by the notorious redox properties of photocatalysts in their excited states, which convert them into highly oxidizing or reducing species, thus producing reactive intermediates that enable degradation of contaminants without the need of chemical strong oxidants or reductants. $^{5}$

However, preparative organic photoredox catalysis in water or aqueous media is not well developed on account of the poor solubility of most organic substrates and catalysts in water or aqueous solvents and difficulties in carrying out photoreactions in heterogeneous media. In this perspective, we describe organic synthetic transformations brought about by visible light photoredox catalysis in water or aqueous media.

Examples where water plays very significant roles in the reaction outcomes are provided. There are occasions where, in its absence, the desired product is not formed at all, or water itself also intervenes as a proton donor or oxygen donor or is necessary in stoichiometric amounts as a coreactant. Even more intriguing effects can be observed when water plays

Received: May 20, 2021

Revised: July 9, 2021 
important roles in the stereoselectivity of the reaction, influencing and driving the formation of products with high diastereoselectivity as opposed to the same reactions performed in organic media. The effect of water for improving enantioselectivity in chiral catalytic photoredox reactions also are studied and explored with representative examples.

In other examples, water is used to solubilize some of the reactants or photocatalysts or employed as a result of a protocol improvement or environmentally friendlier methodology as compared to the same reaction performed in organic solvents. All these combined effects of water as a reaction medium or its very presence in photocatalytic reactions have an ultimate goal which is to diminish the environmental impact of the synthetic and photocatalytic process. There is a recent review article ${ }^{6}$ on visible light-mediated organic transformations in water with special emphasis on the type of photocatalyst (transition metal complexes, organic dyes, solid-supported photocatalysts, semiconductors, and those without photocatalysts).

In this perspective, we describe and emphasize the organic synthetic advantage of carrying out visible light-photocatalyzed reactions in water or aqueous media and classify the perspective into sections according to the synthetic transformation or synthetic goal accomplished. Among the organic transformations enabled by photocatalysis in water or aqueous mixtures, radical additions to carbon-carbon double bonds, aromatic substitution reactions and arylations, decarboxylative reactions, photooxidation and cyclization processes, and miscellaneous reactions are herein described. The mechanisms of the photocatalytic reactions are also given in order to cast some light onto the role of the aqueous medium in the photoreaction. Although this account does not pretend to be an exhaustive review of the literature, representative examples of the above photocatalyzed transformations in water or aqueous media are discussed.

\section{PHOTOCATALYZED SUBSTITUTIONS OF ARENES IN AQUEOUS MEDIA}

Homolytic Aromatic Substitutions with Ulterior Cyclizations. Homolytic aromatic substitution (HAS) is a convenient synthetic strategy to replace aromatic hydrogen atoms by suitable substituents in order to form new $\mathrm{C}-\mathrm{C}$ or $\mathrm{C}$-heteroatom bonds. Even more interesting are HASs ensuing cyclization reactions that form new cyclic scaffolds.

Strategies for the photocatalytic syntheses of phenanthridines in organic media contemplate tandem radical additioncyclization of 2-isocyanobiphenyls and 2-(1-azidovinyl)-1,1'biaryls with diverse radical precursors or the $\mathrm{N}-\mathrm{O}$ bond homolysis cyclization reactions of oxime ethers and oxime esters. $^{7-19}$

A protocol for the preparation of phenanthridine-6carboxylates from $\mathrm{N}$-biarylglycine esters in water was achieved by Natarajan and colleagues. ${ }^{20}$

The authors ${ }^{20}$ found the optimized reaction conditions were 2-([1,1'-biphenyl $]$-2-ylamino $)$ acetate $(0.3 \mathrm{mmol})$ as a substrate and $5 \mathrm{~mol} \%$ of Rose Bengal as a photocatalyst in water under $34 \mathrm{~W}$ blue LED $(\lambda=425 \mathrm{~nm})$ irradiation for $30 \mathrm{~h}$ in the presence of air. In order to explore the substrate scope, the authors $^{20}$ investigated a series of $\mathrm{N}$-biarylglycine esters (substituent effects on the aromatic ring with glycine ester group), as illustrated in Scheme 1.
Scheme 1. Scope of Reaction with Substituted Biaryls

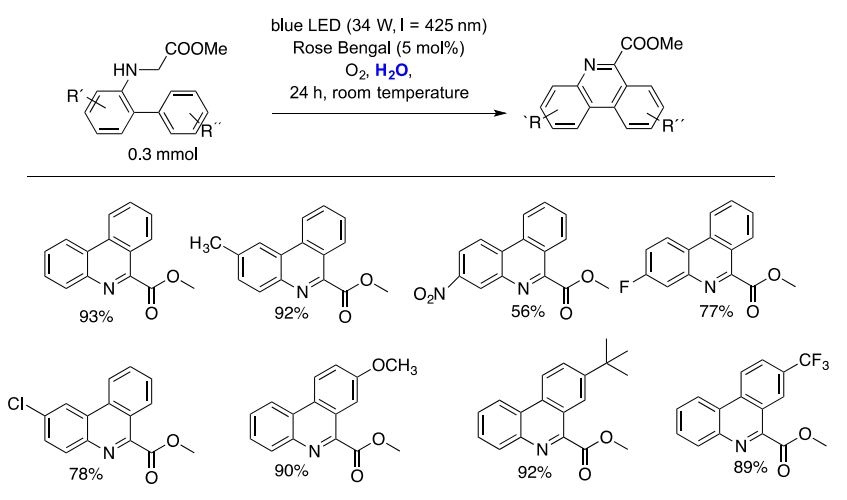

As observed from Scheme 1, both electron-withdrawing and -donating substituents on the biaryl moiety produce high yields of substituted phenanthridines.

The authors ${ }^{20}$ proposed a reaction mechanism such as that depicted in Scheme 2.

\section{Scheme 2. Proposed Reaction Mechanism}

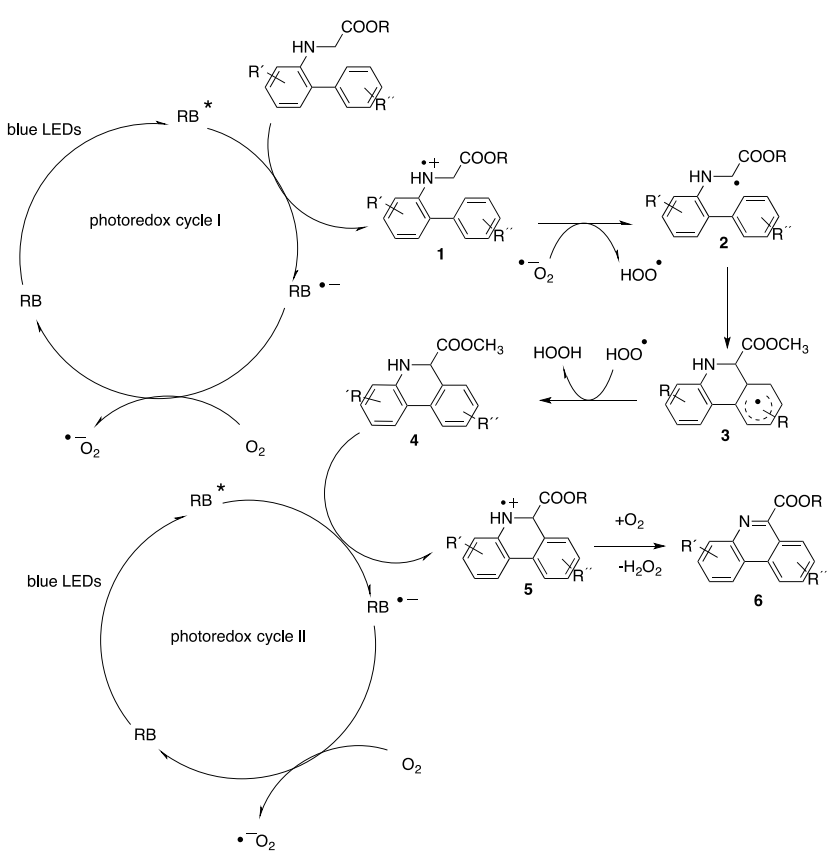

Independent experiments conducted with dihydrophenanthridine 4 (Scheme 2) under irradiation with Rose Bengal as the photocatalyst under the optimized reaction conditions afforded phenanthridine 6 in quantitative yield, purporting that oxidation can occur within the photocatalytic cycle. To start with, excited photocatalyst Rose Bengal $\left(\mathrm{RB}^{*}\right)$ is able to oxidize 2-([1,1'-biphenyl]-2-ylamino)acetate to radical cation 1 yielding the radical anion of $\mathrm{RB}^{-}$which reduces the oxygen present to an anion superoxide. This anion superoxide is, in turn, able to reduce intermediate radical cation $\mathbf{1}$ to radical $\mathbf{2}$, which upon homolytic aromatic substitution renders cyclohexadienyl radical intermediate 3 . Peroxyl radical abstracts a $H$ atom from intermediate 3 rendering dihydrophenanthridine 4 . In a second catalytic cycle, oxidation of 4 takes place to afford phenanthridine $\mathbf{6}^{20}$ 
The construction of other cyclic organic frameworks through photocatalysis has also been reported in water, such as the synthesis of $\mathrm{CF}_{3}$-substituted dihydroisoquinolinones via the photoinduced radical cascade cyclization of $\mathrm{N}$-allylbenzamides with $\mathrm{CF}_{3} \mathrm{SO}_{2} \mathrm{Na}^{6,21}$ (Scheme $3 \mathrm{~A}$ ), the methylsulfonyla-

Scheme 3. Photocatalytic Syntheses of Dihydroisoquinolinones (A), Sulfone-Containing Benzo[A]Fluoren-5-ones (B), Sulfonylated 1-Alkylindolin2-one (C), and Synthesis of Phenanthrols (D)

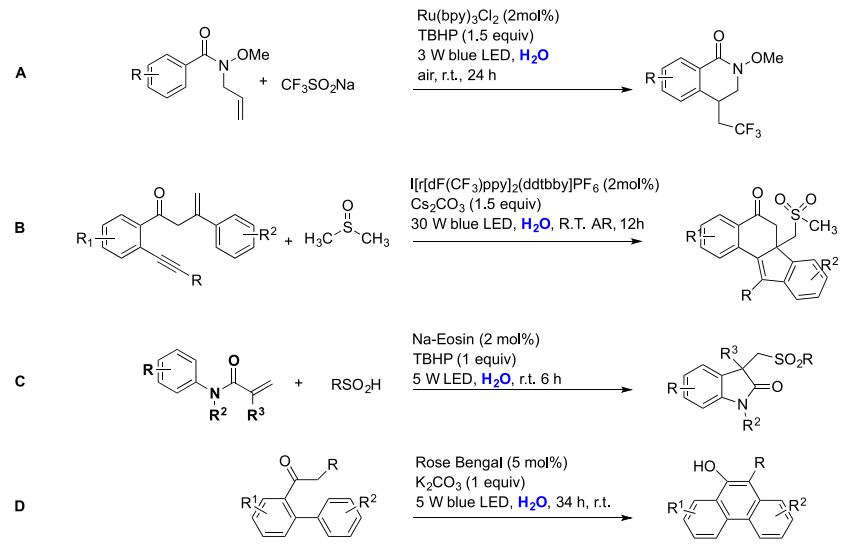

tion/bicyclization of 1,7-enynes with dimethyl sulfoxide for the synthesis of sulfone-containing benzo[a]fluoren-5-ones in the presence of iridium polypyridyl complexes in water ${ }^{6,22}$ (Scheme 3B), the direct preparation of sulfonated oxindoles through the metal-free photocatalytic reactions of $\mathrm{N}$ arylacrylamides and sulfinic acids ${ }^{6,23}$ (Scheme $3 \mathrm{C}$ ), or the synthesis of phenanthrols via the intramolecular cyclization and aromatization reaction of alkyl 1,1'-biaryl-2-ones under Eosin photocatalysis ${ }^{6,24}$ (Scheme 3D). All these reactions have been previously reviewed and are succinctly compiled in Scheme $3 .^{6}$

Arylation Reactions. Radical arylation reactions are synthetic strategies that allow the formation of new $\mathrm{C}_{\mathrm{Ar}}-\mathrm{C}_{\mathrm{Ar}}$ bonds or $\mathrm{C}_{\text {aliph }}-\mathrm{C}_{\mathrm{Ar}}$ bonds from aryl radicals. ${ }^{25}$ There are extensive studies on photocatalyzed arylation reactions. ${ }^{26,27}$ Metal-free photocatalytic arylations ${ }^{28}$ have been reported with numerous photocatalysts, such as carbon nitride-based catalysts. $^{30}$

A few examples of photocatalytic arylations in aqueous media have been informed. ${ }^{31}$

Xue and colleagues ${ }^{32}$ have developed a photocatalytic arylation reaction of electron-deficient heteroarenes in water starting from aryldiazonium salts. The authors ${ }^{32}$ optimized the reaction conditions using tetrafluoroborate 4-methoxybenzenediazonium salt $(0.3 \mathrm{mmol}), \mathrm{Ru}(\mathrm{bpy})_{3} \mathrm{Cl}_{2}$ as the photocatalyst $(2.5 \mathrm{~mol} \%)$, 4-trifluoromethylpyridinium hydrochloride, and an electron-poor heteroaromatic compound $(1.5 \mathrm{mmol})$ as the radical acceptor in water as the solvent (1 $\mathrm{mL}$ ) irradiating with a $40 \mathrm{~W}$ fluorescent bulb for $40 \mathrm{~h}$. Under these conditions, a $76 \%$ yield of 4-(trifluoromethyl-2-(4methoxyphenyl) pyridine was obtained. The authors ${ }^{32}$ proceeded to investigate the reaction scope employing a broad range of heteroarene hydrochlorides and 4-methoxybenzenediazonium salt as the aryl radical precursor, according to Scheme 4.
Scheme 4. Selected Examples for 4-Methoxyphenylation (Ar) of Pyridine Hydrochloride Derivatives

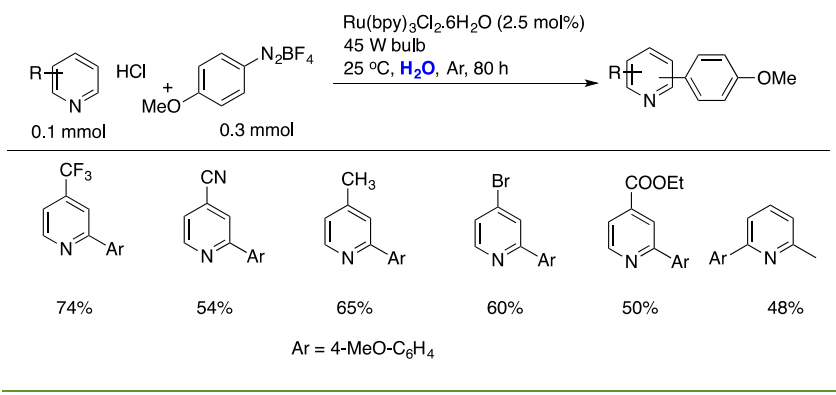

As observed from Scheme 4, pyridines bearing electronwithdrawing groups $\left(\mathrm{CF}_{3}, \mathrm{CN}, \mathrm{COOEt}, \mathrm{Br}\right)$ or electron donors $\left(\mathrm{CH}_{3}\right)$ afforded reasonably good yields of arylated products.

The authors ${ }^{32}$ next evaluated the substrate scope in terms of aryl diazonium salts, substituted with functional groups such as cyano, carboxyethyl, chlorine, bromine, and fluorine employing 4-trifluoromethylpyiridine hydrochloride in water under photocatalysis as the radical acceptor. These transformations and yields are summarized in Scheme 5.

Scheme 5. Selected Examples of Photocatalytic Arylation of 4-Trifluoromethylpyiridine Hydrochloride

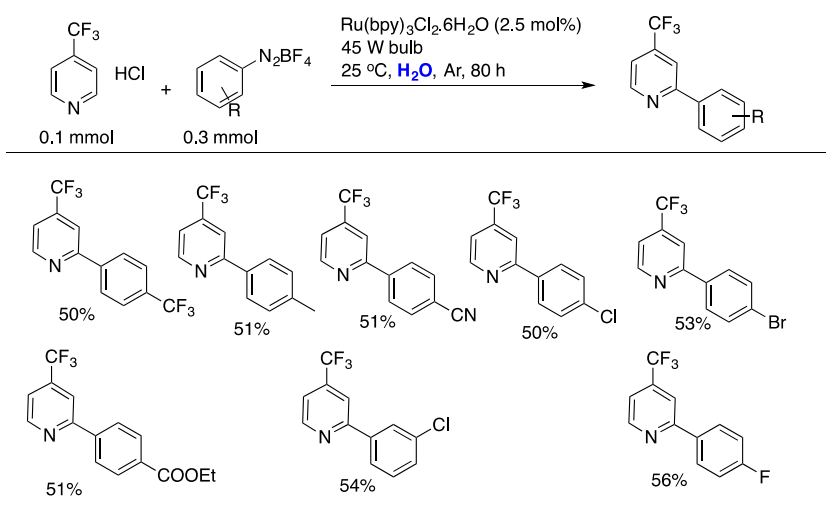

As observed from Scheme 5, the reactivity of different substituted diazonium salts toward the production of the respective aryl radicals is somewhat similar, as contemplated from the arylated product yields.

More complex heterocyclic nuclei, such as caffeine and derivatives, were tested for the arylation scope. However, the reactions under the usual conditions proceeded in very low yields. Upon addition of formic acid, a yield enhancement was observed for these substrates (Scheme 6).

The authors ${ }^{32}$ also attempted a one-pot reaction synthesizing aryldiazonium salt and performing in situ the arylation of the heteroaromatic compound in water, obtaining reasonable yields of the coupling products.

A study of the mechanism through various probe experiments was undertaken. The addition of radical scavenger (2,2,6,6-tetramethylpiperidin-1-yl)oxyl (TEMPO) suppressed the arylation process. On the basis of these latter experiments and literature precedent, the authors postulated a mechanism such as that illustrated in Scheme 7.

To commence, photocatalyst 7 (Scheme 7) is excited through visible light to afford 8 , which through a reductive electron transfer with aryldiazonium salt produces an aryl 
Scheme 6. Selected Examples for Photocatalytic 4-Methoxyphenylation of Caffeine Derivatives in Water
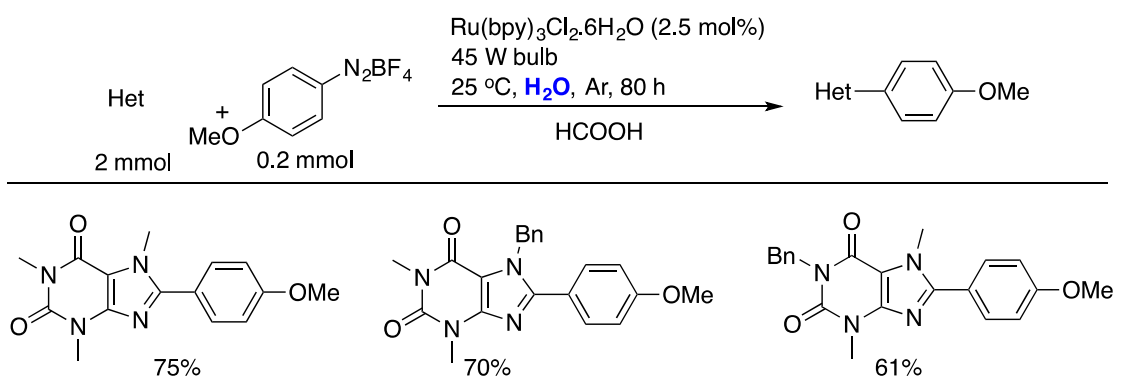

Scheme 7. Proposed Reaction Mechanism

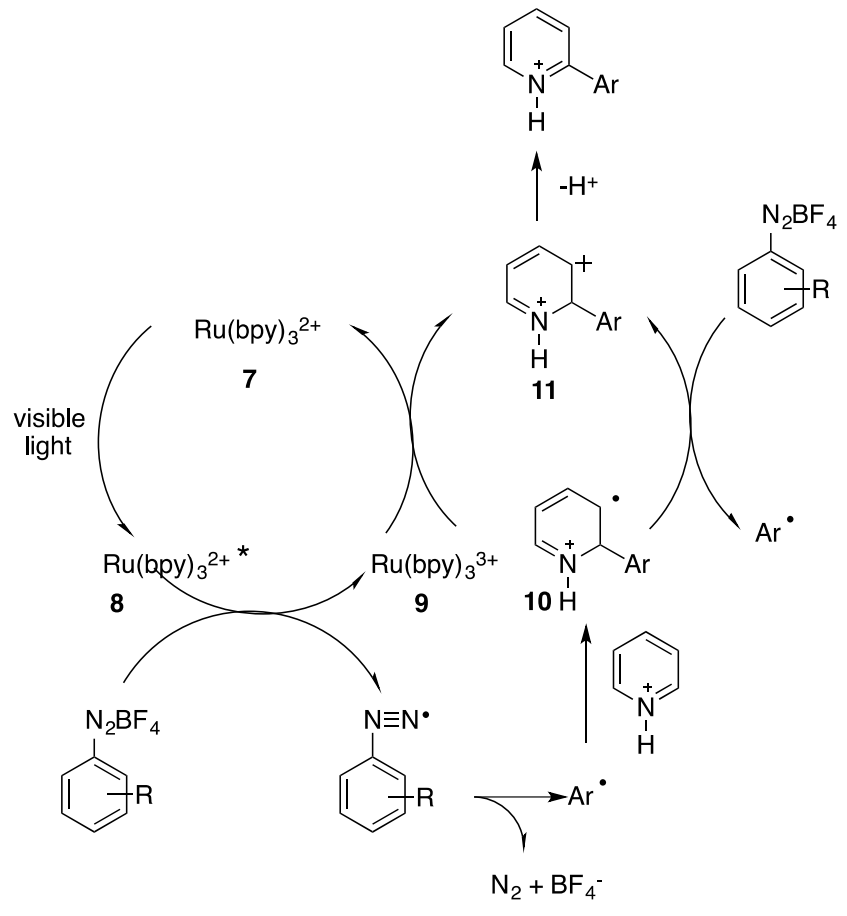

radical, Ar• Homolytic aromatic substitution with a pyridinium chloride gives radical intermediate 10 , which is oxidized by more aryl diazonium salt to intermediate $\mathbf{1 1}$ and aryl radicals which enter the chain cycle. Deprotonation of intermediate 11 affords an arylated pyridine. ${ }^{32}$ Although this reaction is quite simple, and has been carried out in several organic solvents under different radical conditions, the improved methodology presented by the authors in water as the reaction medium and under photocatalysis represents a notorious advantage. As a final remark, the use of water has allowed an improved reactivity of pyridine nuclei as pyridinium salts toward arylation reactions, with the advantages of regioselectivity (substitution of pyridine rings at the 2position), aryl precursors from soluble benzenediazonium salts, and arylation product yields. This methodology employs a standard metal-organic photocatalyst commonly available in laboratories. On the contrary, conventional photocatalyzed arylations of pyridine nuclei afford nonregioselectively substituted pyridine-arylated products in organic solvents. ${ }^{33}$ This is most likely due to different spin densities at the 2positions of pyridines and pyridinium hydrochloride salts.

Wang and collaborators ${ }^{34}$ have disclosed the arylation reactions of heteroarenes, enol acetates, or benzoquinones employing aryldiazonium tetrafluoroborate salts under visible light in an aqueous medium or pure water, a (polyaniline)-g$\mathrm{C}_{3} \mathrm{~N}_{4}-\mathrm{TiO}_{2}{ }^{35-37}$ composite as the photocatalyst, and irradiation with a $14 \mathrm{~W}$ commercial fluorescent lamp (CFL). The scope of the transformation regarding heteroaromatics is depicted in Scheme 8 and that of aliphatic substrates in Scheme 9.

Scheme 8. Scope of Arylation Reaction of Heteroaromatics Employing (Polyaniline)-g- $\mathrm{C}_{3} \mathrm{~N}_{4}-\mathrm{TiO}_{2}$ Photocatalyst

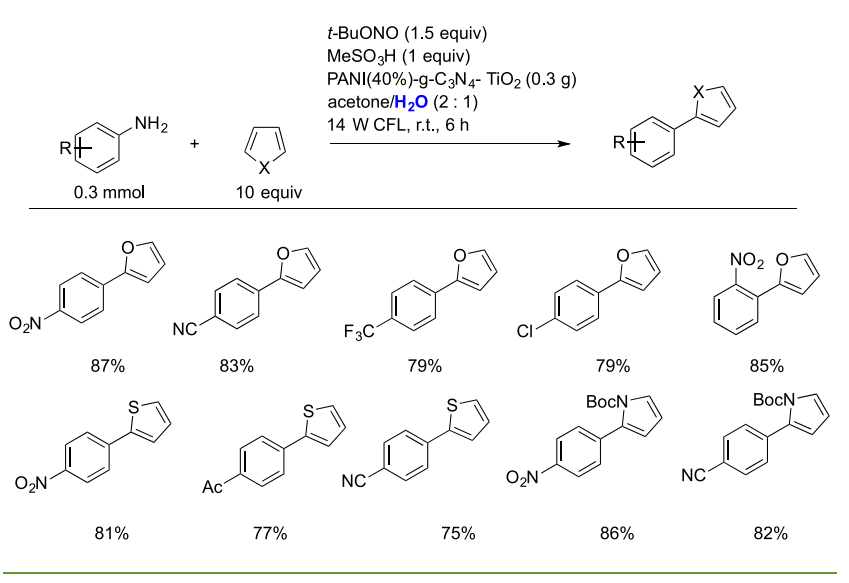

The authors ${ }^{34}$ investigated the reaction mechanism (Scheme 10).

The homolytic decomposition of diazonium salt 12 via an electron transfer (ET) process affords aryl radical 13 (Scheme $10)$. The addition of radical 13 to a heteroarene gives radical

Scheme 9. Scope of Arylation Reaction of Enol Acetates and Benzoquinones Employing (Polyaniline) $-\mathrm{g}-\mathrm{C}_{3} \mathrm{~N}_{4}-\mathrm{TiO}_{2}$ Photocatalyst

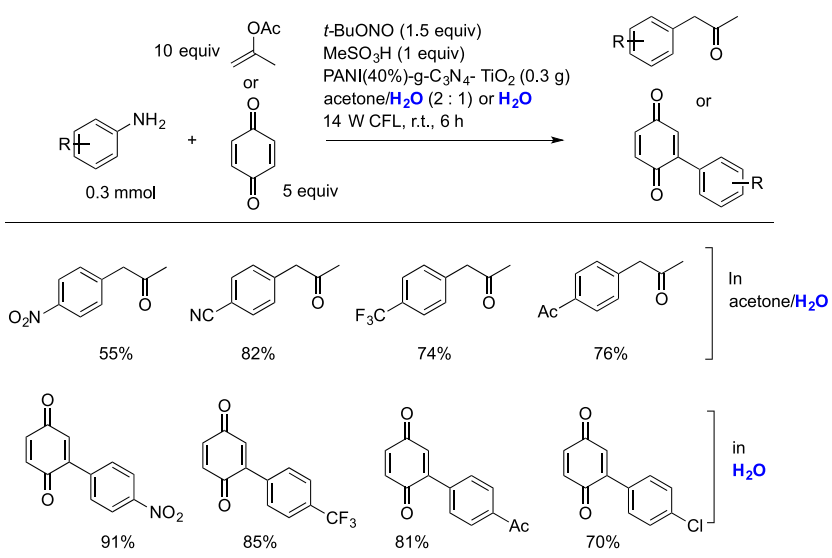




\section{Scheme 10. Proposed Reaction Mechanism}
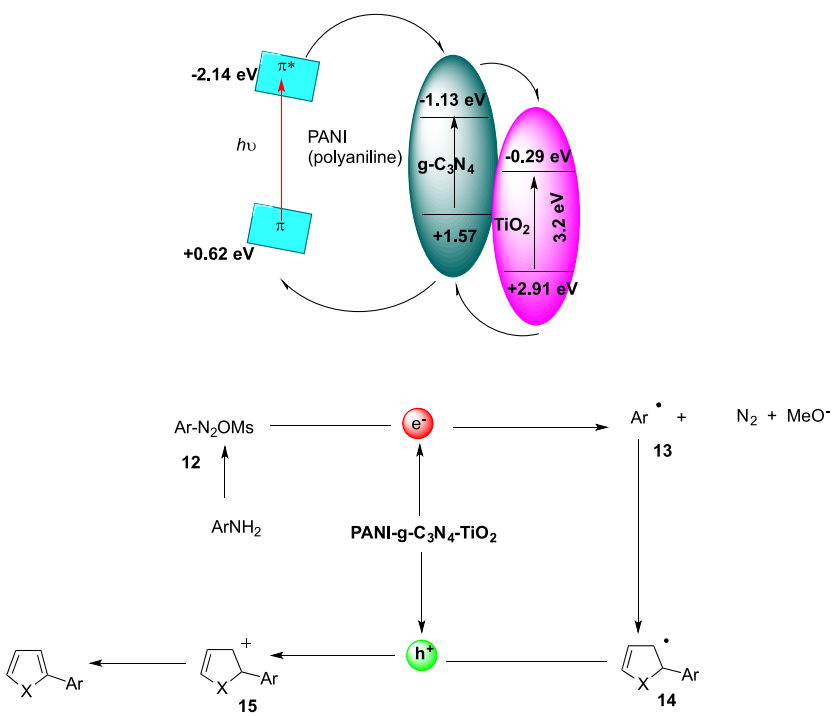

14, which is further oxidized by the photogenerated holes $\left(h^{+}\right)$ to form carbocation intermediate 15. Finally, intermediate $\mathbf{1 5}$ is deprotonated to deliver the desired product. In the catalytic mode, the potentials of PANI ( $\pi^{*}$-orbital, $-2.14 \mathrm{eV} ; \pi$-orbital, $+0.62 \mathrm{eV})$ are more negative than the valence band (VB) and conduction band $(\mathrm{CB})$ potentials of $\mathrm{g}-\mathrm{C}_{3} \mathrm{~N}_{4}(-1.13$ and +1.57 $\mathrm{eV}$, respectively) as well as those of $\mathrm{TiO}_{2}(-0.29$ and $+2.91 \mathrm{eV}$, respectively). Consequently, the excited electrons migrate to the $\mathrm{CB}$ of $\mathrm{TiO}_{2}$, while the holes are gathered by the $\pi$-orbital of PANI. In this manner, the electron and hole are well separated, preventing the recombination process. ${ }^{34}$

Next, a comparison between different photocatalyzed protocols for the arylation reactions of small heterocycles is shown in Table 1.

As observed from Table 1, yields of products under photocatalysis in water are slightly higher than those obtained in organic solvents, with the regioselectivity as good in water as in organic solvents.

\section{RADICAL ADDITIONS TO CARBON-CARBON MULTIPLE BONDS IN AQUEOUS MEDIA}

Alkylation of Alkenes. Addition of a saturated hydrocarbon $(\mathrm{R}-\mathrm{H}$ or $\mathrm{R}-\mathrm{X})$ to an olefin to yield a saturated hydrocarbon of higher molecular weight is known as alkylation. Such reactions are employed to produce medium molecularweight scaffolds from smaller molecules. ${ }^{38}$ These processes can be performed through the intervention of metals, ${ }^{39,40}$ photocatalysts, $^{41-43}$ or electrocatalysis. ${ }^{44,45}$ In general, radical 1,2difunctionalyzation reactions of alkenes are quite general, and several photocatalytic methods have been employed. ${ }^{46}$

Tetrabutylammonium decatungstate $(\text { TBADT })^{47}$ is considered a competent and functional photocatalyst for hydrogen atom transfer (HAT) reactions that make possible the scission of both activated and unactivated aliphatic $\mathrm{C}-\mathrm{H}$ bonds. ${ }^{48,49}$

Bonassi and colleagues ${ }^{50}$ have found a decatungstatephotocatalyzed alkylation of electron-deficient alkenes under continuous flow conditions. The optimized reaction conditions consist of the use of tetrabutylammonium decatungstate (TBADT, $0.4-2 \mathrm{~mol} \%$ ) as the photocatalyst, substrate of alkylbenzene $(0.5 \mathrm{M}), 1,2$-dicyanoethylene $(0.1 \mathrm{M})$ as the radical acceptor, $\mathrm{LiClO}_{4}(0.5 \mathrm{M})$ in $\mathrm{MeCN}$ :water $(50 \mathrm{~mL}, 5: 1)$ as the solvent mixture in a photochemical flow apparatus, and irradiation with a $500 \mathrm{~W} \mathrm{Hg}$ vapor lamp. The scope of the reaction is illustrated in Scheme 11.

Scheme 11. Selected Examples of Alkylation of ElectronDeficient Alkenes

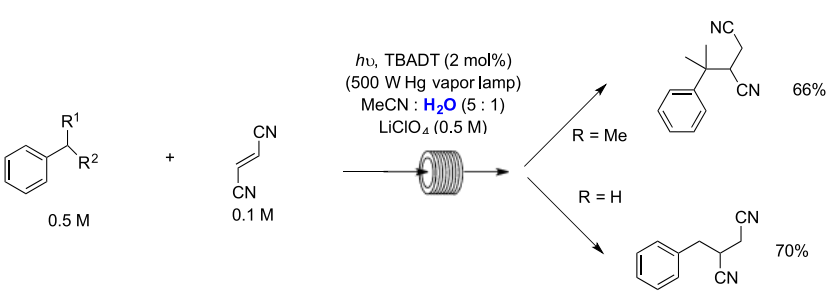

The use of a continuous flow approach enables a decrease of the residence time and an increase of the productivity with respect to batch processes. The concentrations of the

Table 1. Comparison between Photocatalyzed Protocols for Arylation of Small Heterocycles in Water (Column 2, This Perspective) and Organic Solvent (Column 3, Other Authors)

\begin{tabular}{|c|c|c|}
\hline PRODUCT & $\begin{array}{c}\text { Product \% from } \\
\text { photocatalysis in water } \\
\text { (Scheme 8) }\end{array}$ & $\begin{array}{c}\text { Product \% from } \\
\text { photocatalysis in organic } \\
\text { solvents }\end{array}$ \\
\hline $74,{ }^{\text {a }} 71^{\mathbf{b}}$ \\
\hline
\end{tabular}

${ }^{a}$ Reference 28. In DMSO as the solvent, with Eosin Y as the photocatalyst. ${ }^{b}$ Reference 29. In DMSO as the solvent, with cercosporin as the photocatalyst. 
substrates can be increased under flow conditions, leading to an improvement of the sustainability of the process. ${ }^{50}$

Chen and collaborators ${ }^{51}$ developed a photocatalyzed radical methodology using water as the cosolvent for achieving the hydroxytrifluoroethylation of styrenes. In a typical reaction, $f a c-\operatorname{Ir}(\mathrm{ppy})_{3}$ is employed as the photoredox catalyst, with the reaction medium being a mixture of acetonitrile:water $(8: 1)$ in the presence of $\mathrm{N}, \mathrm{N}$-diisopropylethylamine as the reductive quencher under an oxygen atmosphere. Water plays a very significant role in the reaction outcome because in its absence the desired product is not formed. The authors ${ }^{51}$ investigated the scope of different styrene derivatives including styrenes in the presence of electron-donating or halogen substituents; in all cases, the reaction is well tolerated as shown in Scheme 12.

Scheme 12. Selected Examples for Hydroxytrifluoroethylation of Styrenes by Photoredox Catalysis Employing a Mixture of $\mathrm{MeCN}: \mathrm{H}_{2} \mathrm{O}(8: 1)$ as Reaction Media

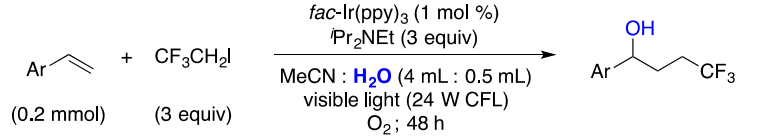

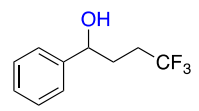

$48 \%$<smiles>OC(CCC(F)(F)F)c1ccc(Br)cc1</smiles><smiles>COc1ccc(C(O)CCC(F)(F)F)cc1</smiles><smiles>OC(CCC(F)(F)F)c1ccc(Cl)cc1</smiles>

$71 \%$<smiles>Cc1ccc(C(O)CCC(F)(F)F)cc1</smiles>

$78 \%$

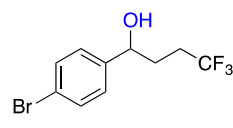

$55 \%$<smiles>OC(CCC(F)(F)F)c1ccc(-c2ccccc2)cc1</smiles>

$68 \%$

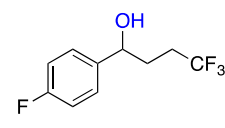

$74 \%$

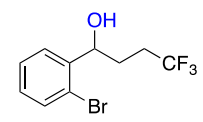

$46 \%$
On the basis of different probe experiments, the authors proposed a reaction mechanism ${ }^{51}$ (Scheme 13) where initially the photocatalyst $f a c-\mathrm{Ir}^{\mathrm{III}}(\mathrm{ppy})_{3}$ is promoted to an excited state which shows a metal-to-ligand charge transfer character affording $f a c-\mathrm{Ir}^{\mathrm{IV}}(\mathrm{ppy})_{2}(\mathrm{ppy})^{\bullet-}$ which is further reduced by $\mathrm{N}, \mathrm{N}$-diisopropylethylamine generating $\mathrm{fac}-\mathrm{Ir}^{\mathrm{III}}(\mathrm{ppy})_{2}(\mathrm{ppy})^{\bullet-}$. A $\mathrm{CF}_{3} \mathrm{CH}_{2} \mathrm{I}$ molecule is reduced by $f a c-\mathrm{Ir}^{\mathrm{III}}(\mathrm{ppy})_{2}(\mathrm{ppy})^{\bullet-}$ regenerating the photocatalyst into its active photoredox state and affording $\left[\mathrm{CF}_{3} \mathrm{CH}_{2} \mathrm{I}\right]^{\bullet-}$ which rapidly yields $\mathrm{I}^{-}$and a trifluoroethyl radical $\left(\mathrm{CF}_{3} \mathrm{CH}_{2}{ }^{\circ}\right)$ which is trapped by the styrene derivative affording intermediate 16 (Scheme 13). Under the presence of oxygen and water, radical intermediate 16 (Scheme 13) reacts with molecular oxygen and yields a hydroxytrifluoroethylation reaction product (Scheme 13). ${ }^{51}$

Although water participates in the reaction outcome providing the hydroxyl functionality in the $\beta$-trifluoromethylated alcohol, its absence, as stated before, does not form any reaction product, not even the atom transfer radical addition (ATRA, vide infra) iodinated product is observed.
Scheme 13. Proposed Reaction Mechanism for Hydroxytrifluoroethylation of Styrenes by Photoredox Catalysis Employing a Mixture of $\mathrm{MeCN} \mathrm{H}_{2} \mathrm{O}(8: 1)$ as Reaction Media

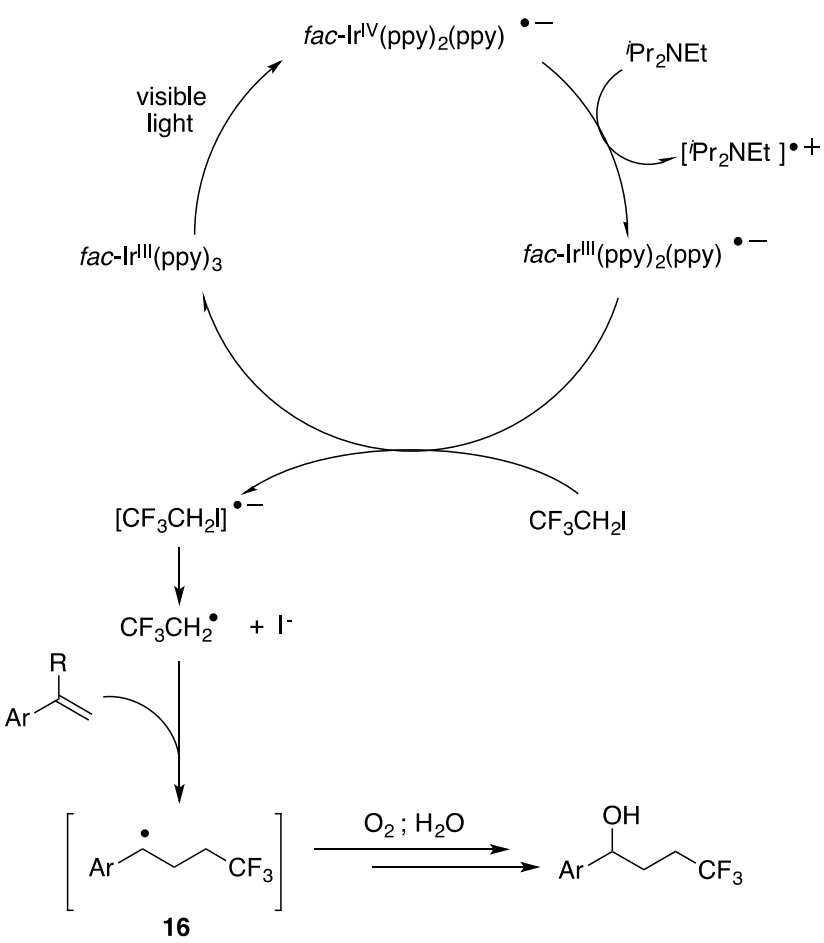

Enantioselective radical conjugate additions have been carried out with chiral inductors such as Al-Salen in DCM as the solvent ${ }^{52}$ or other chiral inductors in organic media. ${ }^{53}$

Meggers and collaborators ${ }^{54}$ developed a photocatalytic addition of alkyl radicals to alkenes with high enantioselectivity using mixtures of acetone and water as reaction media. In a typical reaction setup, the authors employed organotrifluoroborates as alkyl radical precursors, $\alpha, \beta$-unsaturated acyl imidazoles (or pyrazoles) as substrates, bis-cyclometalated rhodium as the chiral Lewis acid catalyst, and 9-mesityl-10methyl-acridinium perchlorate $\left(\mathrm{Mes}-\mathrm{AcrClO}_{4}\right)$ as the photosensitizer under blue LED irradiation. ${ }^{54}$ Regarding the organotrifluoroborate radical precursor, the reaction worked well with benzyltrifluoroborates with electron-donating and -withdrawing substituents, many alkoxylmethyltrifluoroborates, and secondary and tertiary alkyltrifluoroborates. Regarding the $\alpha, \beta$-unsaturated acyl imidazoles alkene substrates, the scope of the reaction was extended to aliphatic substituents, either electron-rich or electron-deficient aromatic moieties; some selected examples are shown in Scheme 14. ${ }^{54}$

The authors ${ }^{54}$ proposed the reaction mechanism shown in Scheme 15 based on several experiments including SternVolmer plots, radical trapping experiments, and reactions carried out in the presence of $\mathrm{D}_{2} \mathrm{O}$ as the cosolvent. The alkyl radical source is potassium benzyltrifluoroborate. The reaction is initiated upon illumination of mesityl acridinium photocatalyst (path a), followed by a SET process with potassium benzyltrifluoroborate (path $b$ ) to yield a benzyl radical that adds to the $N, O$-rhodium-coordinated $\alpha, \beta$-unsaturated 2 -acyl imidazole (intermediate 17, Scheme 15, path c) substrate generating a secondary radical 18 (Scheme 15). Radical intermediate 18 (Scheme 15) is reduced by a single electron 
Scheme 14. Selected Examples for Catalytic

Enantioselective Addition of Alkyl Radicals to Alkenes by Visible Light-Activated Photocatalysis Using Mixtures of Acetone and Water as Reaction Media

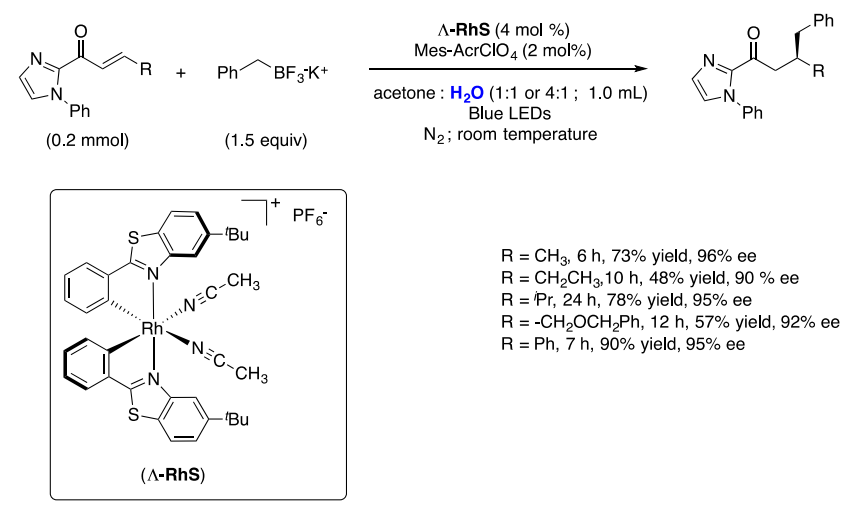

transfer process from the reduced form of the photocatalyst (path d), affording a rhodium enolate (intermediate 19, Scheme 15) and regenerating the photocatalyst. Finally, a water molecule protonates intermediate 19 (Scheme 15) yielding a rhodium-bound product (intermediate 20, Scheme 15). In fact, the role of water is to form rhodium-bound intermediate $\mathbf{2 0}$ which, by protonation from water, weakens the $\mathrm{Rh}-\mathrm{O}$ bond and collapses to product-regenerating intermediate $\mathbf{1 7}$ that re-enters the cycle (i.e., protonation of the $\mathrm{C}$-enol bond). It must be outlined that the coordination of rhodium with the substrate enhances the rate of radical addition by a factor of $3 \times 10^{4}$; this means that the radical addition to a rhodium-coordinated substrate is much faster than the addition to the free substrate, allowing for the high enantioselectivity observed for the reaction. ${ }^{54}$

Atom Transfer Radical Addition (ATRA) to Olefins. Photocatalyzed atom transfer radical additions (PhATRAs) to olefins have been found with metal photocatalysts, such as the polypyridyl complexes of $\mathrm{Ir}^{55,56}$ and $\mathrm{Ru}$-based photocatalysts, ${ }^{56-58} \mathrm{Bi}_{2} \mathrm{O}_{3}$, ${ }^{59,60} \mathrm{Cu}$-derived complexes activated by light, ${ }^{61-63} \mathrm{TiO}_{2}{ }^{64}$ and $\mathrm{Au}$ complexes ${ }^{65}$ in organic solvents. Nonmetal-organic photocatalysts, such as 4-methoxybenzaldehyde, ${ }^{66,67}$ and light-activated halogen-bonding complexes $^{68,69}$ have also been reported to perform ATRA reactions. Pyrene-based metal organic frameworks have been found to achieve ATRA products. ${ }^{70}$ Copper-mediated thermal reactions have also been studied for ATRA processes. ${ }^{71,72}$

A seminal paper on photocatalyzed ATRA reactions in aqueous media has been advanced by Stephenson and colleagues. ${ }^{56}$ The report by Stephenson and collaborators ${ }^{73}$ can be considered the first found intermolecular ATRA reaction of haloalkanes and $\alpha$-halocarbonyls with olefins by photoredox catalysis activated by visible light in aqueous media. ${ }^{74}$ The authors ${ }^{73}$ found optimal reaction conditions when employing $\operatorname{Ir}\left[\mathrm{dF}\left(\mathrm{CF}_{3}\right) \mathrm{ppy}\right]_{2}(\mathrm{dtbbpy}) \mathrm{PF}_{6}$ as a photocatalyst, $1 \mathrm{mmol}$ of olefin, 2 equiv of the atom transfer agent, $\mathrm{LiBr}$ as the additive, a mixture of DMF: $\mathrm{H}_{2} \mathrm{O}(1: 4)$ as the reaction medium under Ar atmosphere, and irradiation with blue LEDs (Scheme 16). The reaction scope is extended to several olefins with different functional groups including alcohols, benzyl esters, alkyl bromides, silyloxy esters, esters, enones, carbamates, and aromatic rings (Scheme 16). Regarding the atom transfer agent, the reaction worked well with various haloalkanes and $\alpha$-halocarbonyls, including $\mathrm{CF}_{3} \mathrm{I}^{73}$

On the basis of several studies, the authors ${ }^{73}$ proposed a reaction mechanism (Scheme 17) starting with the photoexcitation of the $[\operatorname{Ir}(\mathrm{III})]$ photocatalyst to afford $* \operatorname{Ir}\left[\mathrm{dF}\left(\mathrm{CF}_{3}\right)\right.$ $\mathrm{ppy}_{2}(\mathrm{dtbbpy})^{+}\left[\mathrm{Ir}(\mathrm{III})^{*}\right]$ that undergoes a single electron transfer process with the haloalkane (or $\alpha$-halocarbonyl) generating $\operatorname{Ir}\left[\mathrm{dF}\left(\mathrm{CF}_{3}\right) \text { ppy }\right]_{2}(\mathrm{dtbbpy})^{2+}[\operatorname{Ir}(\mathrm{IV})]$ (bearing a halide as a counterion) and the electrophilic alkyl radical 21 (Scheme 17). Alkyl radical 21 (Scheme 17) adds to the olefin

Scheme 15. Proposed Reaction Mechanism for Catalytic Enantioselective Addition of Alkyl Radicals to Alkenes by Visible Light-Activated Photocatalysis Using Mixtures of Acetone and Water as Reaction Media

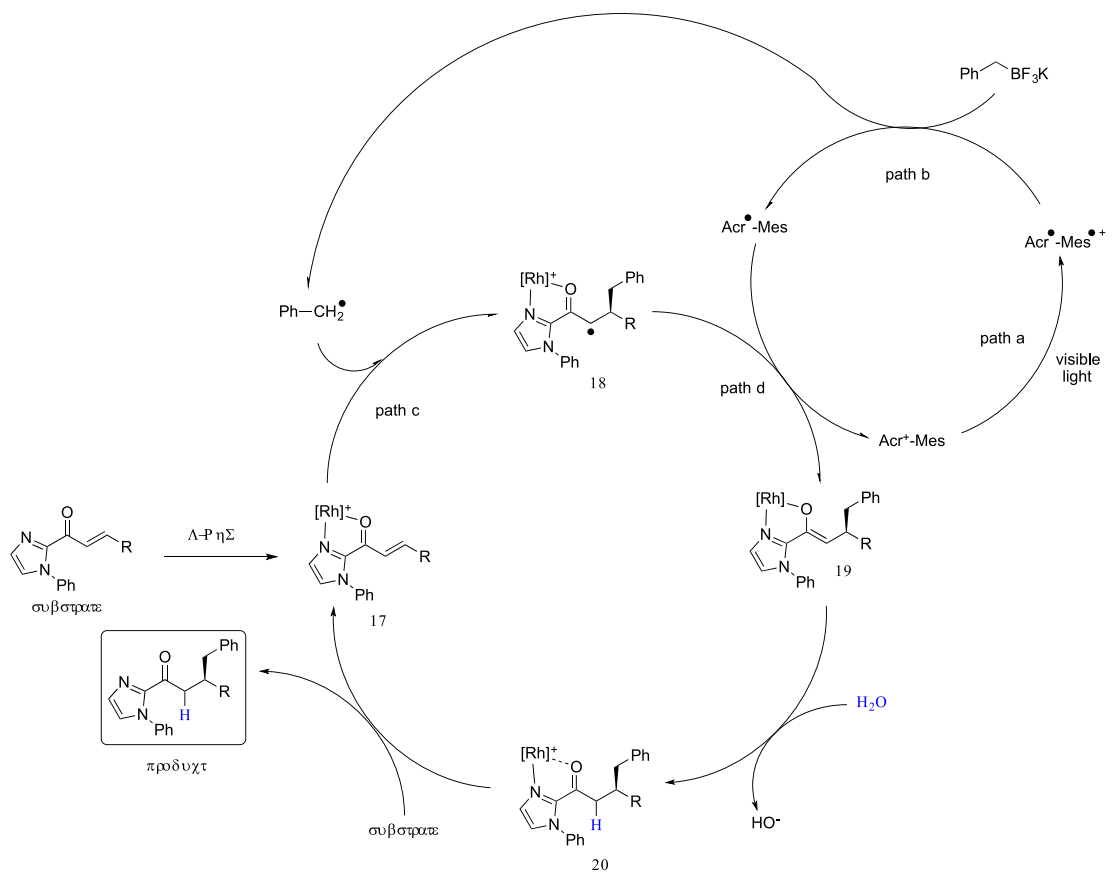


Scheme 16. Selected Examples for Intermolecular Atom Transfer Radical Addition (ATRA) to Olefins by Photoredox Catalysis in Aqueous Media Proposed by Stephenson and Collaborators ${ }^{73}$

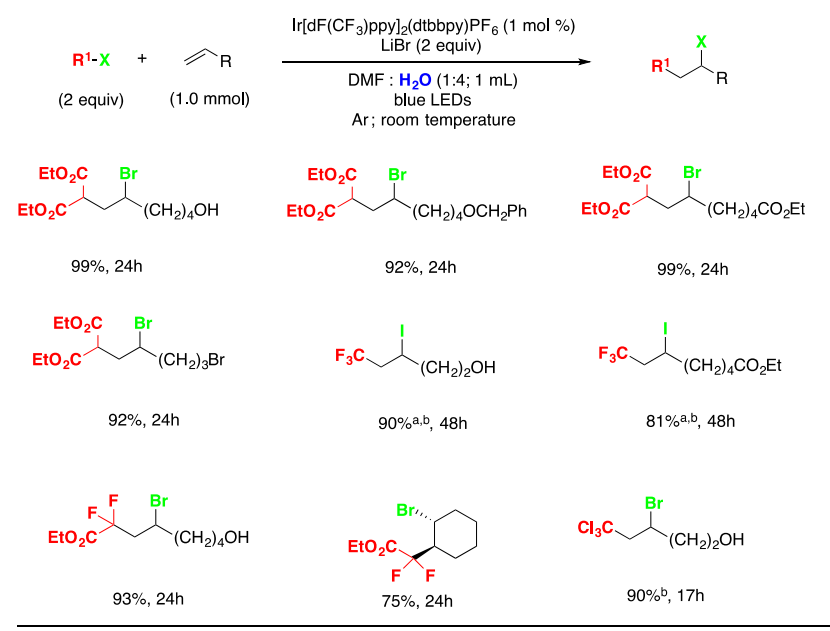

a excess of haloalkane used

${ }^{\mathrm{b}}$ no LiBr added

Scheme 17. Proposed Reaction Mechanism for

Intermolecular Atom Transfer Radical Addition (ATRA) to Olefins by Photoredox Catalysis in Aqueous Media Proposed by Stephenson and Collaborators ${ }^{73}$

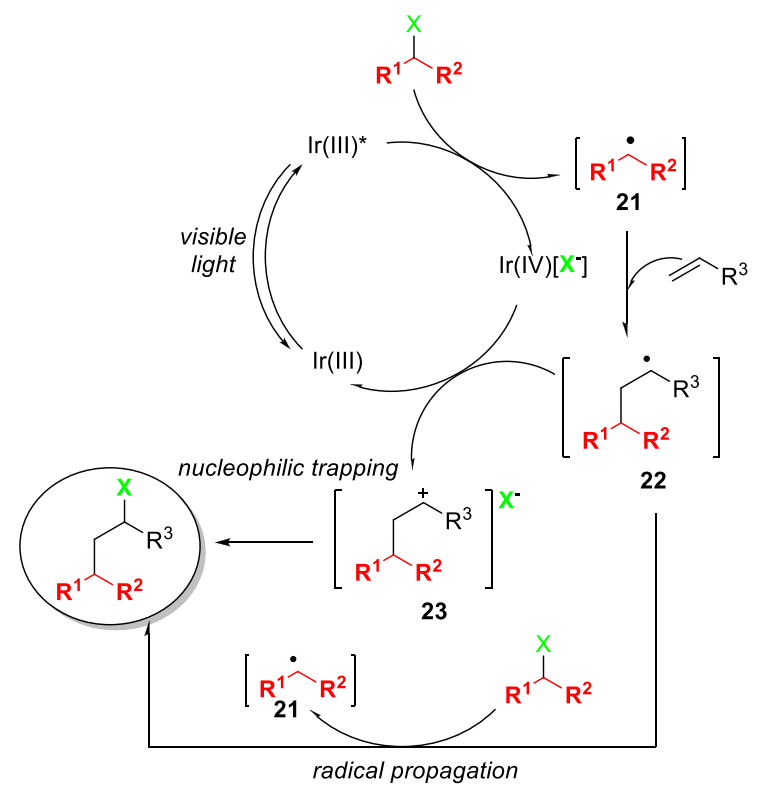

forming radical adduct $\mathbf{2 2}$ (Scheme 17). The formation of the ATRA reaction product is possible by two different pathways. One possibility contemplates the oxidation of radical adduct 22 (Scheme 17) by $[\operatorname{Ir}(\mathrm{IV})]$ preassociated with the halide counterion, giving carbocation $\mathbf{2 3}$ (Scheme 17) that rapidly combines with the halide anion to yield the reaction product. It must be noted that external nucleophiles, including water (major cosolvent), do not react with proposed carbocation intermediate $\mathbf{2 3}$ (Scheme 17) as observed in the mechanistic experiments $^{73}$ performed by the authors. The second possibility for obtaining the ATRA reaction product involves a radical chain transfer mechanism where radical intermediate 22 (Scheme 17) abstracts a halide atom from a haloalkane (or a-halocarbonyl) molecule giving the reaction product and alkyl radical 21 (Scheme 17) that can propagate the reaction. ${ }^{73}$ The absence of oxygen or any other external oxidant probably prevents oxidation of intermediate $\mathbf{2 1}$ to a secondary carbocation, precluding the formation of the hydroxylic product, as observed from Scheme 12 (vide supra).

In another report, ${ }^{56}$ the authors further exploited ATRA reactions in aqueous media with $\left[\mathrm{Ru}(\mathrm{bpy})_{3}\right] \mathrm{Cl}_{2}$ as the photocatalyst in DMF:water as the solvent mixture, affording products in reasonable yields.

A metal-free iodoperfluoroalkylation of alkenes employing Eosin $\mathrm{Y}$ as the photocatalyst $(1 \mathrm{~mol} \%)$ and aqueous $\mathrm{Na}_{2} \mathrm{~S}_{2} \mathrm{O}_{3}$ as the electron donor in the presence of perfluoroalkyl iodides has been found by Yajima and Ikegami. ${ }^{75}$ Selected examples of the transformation are depicted in Scheme 18.

Scheme 18. Selected Examples for Iodoperfluoroalkylation of Alkenes in Aqueous Media

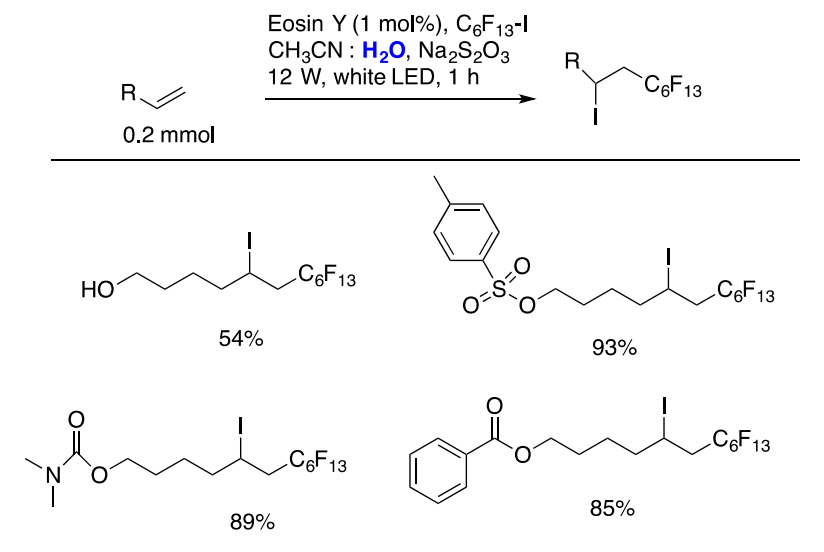

Through a series of experiments (use of TEMPO and luminescence quenching experiments), the authors, ${ }^{75}$ proposed the following reaction mechanism in Scheme 19.

Scheme 19. Proposed Mechanism

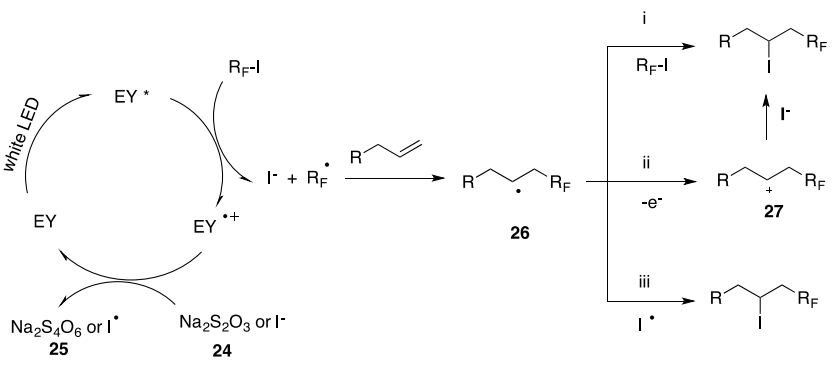

In the proposed mechanism (Scheme 19), visible lightexcited Eosin Y reduces the perfluoroalkyl iodide $R_{F}-I$ to $R_{F}$ radicals. The resulting oxidized Eosin $\mathrm{Y}$ (radical cation of Eosin $\mathrm{Y}$ ) is reconverted to a photoactive catalyst in the presence of 24 (affording $\mathrm{Na}_{2} \mathrm{~S}_{4} \mathrm{O}_{6}, 25$ ) or iodide (giving iodine radical). The reaction of $R_{F}$ radicals with olefin renders intermediate radical 26, which is converted to product by either one of the three routes available, that is, (i) abstraction of iodine from $\mathrm{R}_{\mathrm{F}}-\mathrm{I}$, (ii) oxidation of intermediate radical 26 to carbocation 27 and ulterior iodide nucleophilic attack, or (iii) recombination of radical intermediate $\mathbf{2 6}$ with radical iodine, to afford the iodoperfluoroalkylated product. The nucleophilic route was 
ruled out, as no product derived from a nucleophilic water attack on carbocation 25 was encountered. The authors, ${ }^{75}$ however, discarded the radical chain possibility (i.e., 26 abstracted an iodine radical from $\mathrm{R}_{\mathrm{F}}-\mathrm{I}$ to propagate the chain, path (i)) on the basis of a light on/off experiment, where product formation is interrupted when irradiation is off (purporting that no radical chain is maintained). However, typical radical chain processes normally take place on the second or subsecond time scale, and so, the observation that product conversion is discontinued in the absence of light could also be in agreement with chain processes that cease faster than the time scale employed in the analytical measurement. ${ }^{76}$

Prato and colleagues ${ }^{77}$ have also reported on the photocatalyzed perfluoroalkylation of terminal olefins in $\mathrm{MeCN} /$ water mixtures employing perylene diimides as the photocatalyst and sodium ascorbate as the sacrificial donor, with perfluoroalkyl iodides as the source of perfluoroalkyl radicals, giving affordable yields of iodoperfluoroalkanes.

The addition of radicals to silyl ketenes has been studied in the past through thermal radical methods. ${ }^{78}$ Acyl radicals, in turn, have been generated from $\alpha$-ketoacids, aldehydes, anhydrides, acyl thioesters, carboxylic acids, ${ }^{79}$ acyl silanes, or acyl chlorides by photocatalysis in organic solvents. ${ }^{80}$

Zhang and colleagues ${ }^{81}$ have reported a sequential protocol in which a photoredox catalytic reaction is placed in sequence with asymmetric transfer hydrogenations of aryl ketones to provide different chiral alcohols with high enantioselectivity in aqueous mixtures of organic solvents. Although these reactions make use of water, water itself is not involved in the photocatalysis.

Sulfonylation of Vinylcyclopropanes. Radical 1,3functionalyzations leading to 1,2,3-trisubstituted alkanes have been reported. ${ }^{82}$ In turn, 1,4-difunctionalyzations have been achieved with alkyl diacyl peroxides or $\mathrm{N}$-fluorobenzenesulfonimide. ${ }^{83}$ These processes have been carried out in organic solvents.

1,5-Difunctionalyzations involving both the introduction of a fluoroalkylthio and a sulfone group are rare. One such example was provided by Liu and collaborators ${ }^{84}$ who developed an environmentally friendly 1,5-trifluomethylthio-sulfonylation of vinylcyclopropanes catalyzed by Eosin Y under visible light in a water phase. The optimum reaction conditions were obtained when $\alpha$-cyclopropyl styrene suspended in water was subjected to the Eosin Y-photocatalyzed reaction in the presence of SDS and $S$-(trifluoromethyl)-4-chlorobenzenesulfonothioate (28, Scheme 20) under white LED irradiation, obtaining a $76 \%$ yield of product (29, Scheme 20) and 7:1 stereoselectivity (Z:E) (Scheme 20). ${ }^{84}$

The authors ${ }^{84}$ were intrigued by the high $Z$ stereoselectivity obtained in water (i.e., 7:1), as opposed to the reaction

Scheme 20. Optimized Reaction Conditions for 1,5Trifluomethylthio-sulfonylation of Vinylcyclopropanes Catalyzed by Eosin Y under Visible Light in Water Phase

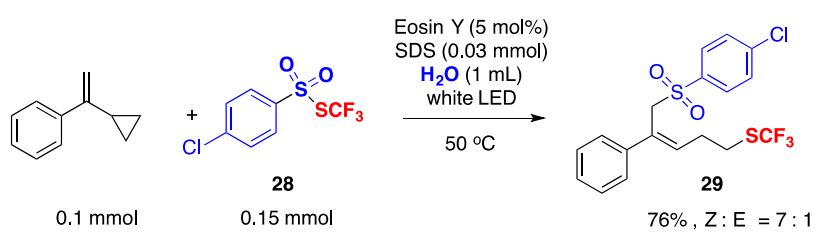

performed in DMSO or DMF organic solvents. The stereoselectivity was found to improve with temperature, obtaining best results at $50{ }^{\circ} \mathrm{C}$. They inspected the electronic effects and steric hindrance in the trifluoromethylthiosulfonylating reagent. When $S$-(trifluoromethyl) 4-(tert-butyl) benzenesulfonothioate 30 (Scheme 21) was employed as difunctionalization

Scheme 21. Selected Examples for 1,5-Trifluomethylthiosulfonylation of Vinylcyclopropanes
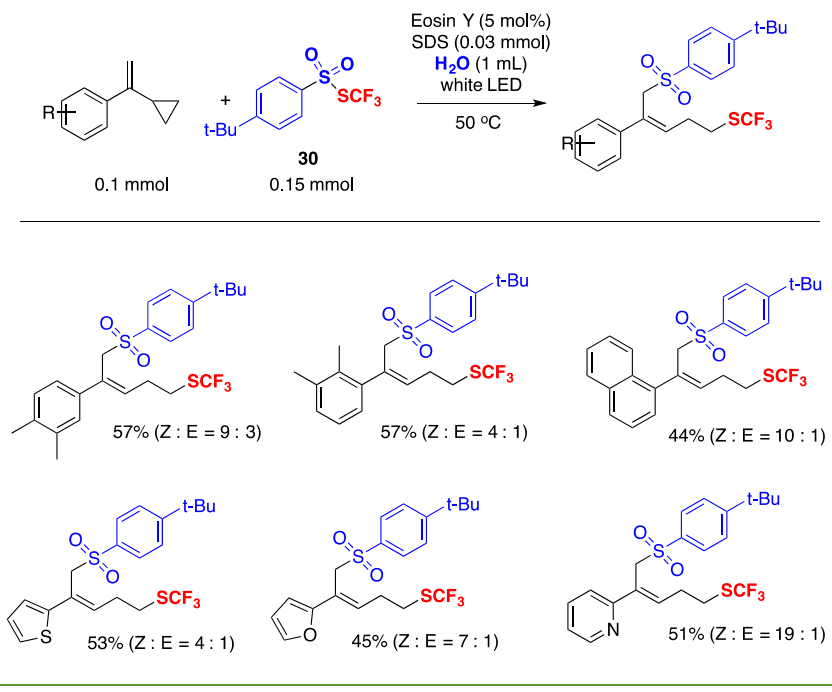

reagent, an improved 13:1 stereoselectivity ratio was achieved in favor of the $Z$ stereoisomer. The reasons for this preference are not given in the original publication but probably are related to a stabilized six-membered ring transition state structure for the incipient $Z$-isomer in water as compared to organic solvents. Having established the best reaction conditions, the authors ${ }^{84}$ explored the scope of the reaction, as summarized in Scheme 21.

Para-substituted substrates achieved good isolated yields of products with very high $Z / E$ ratios (Scheme 21). ${ }^{84}$ The authors performed some control experiments. When TEMPO was used, the reaction was completely inhibited, and reagent 30 disappeared. Also, substrate cyclopropyl styrene remained. These results indicated that $\mathbf{3 0}$ is initiated first, and the arylsulfonyl radical thus regenerated adds to the styryl double bond ensuing a terminal carbon radical which is terminated with the trifluoromethylthio radical. The authors postulated a reaction mechanism such as that shown in Scheme $22 .^{84}$

Initially, upon visible light excitation of Eosin Y, a photoactive triplet state of the photocatalyst is generated (Scheme 22), which by ET to $S$-(trifluoromethyl)-arylsulfonothioate reagent 31 generates arylsulfonyl radical 32, which adds to styrylcycyclopropane 33 to give radical intermediate 34, which upon reaction with complex $\left[\right.$ Eosin $\left.\mathrm{Y} / \mathrm{SCF}_{3}\right]$ affords product 35 (Scheme 22). ${ }^{84}$ The role of the $\mathrm{MeCN} /$ water (SDS) mixture is only an alternative to a classic all-organic solvent medium, such as that reported in the protocol for the photocatalyzed reaction carried out in 1,4-dioxane. ${ }^{85}$

Arylation of Alkenes. Direct arylation of alkenes has been achieved by the $\mathrm{Ru}(\mathrm{IV})$ catalyst $^{86,87}$ and by copper catalysis. ${ }^{88}$

In 2014, König and colleagues ${ }^{89}$ found the photocatalyzed arylation of styrene derivatives with tetrafluoroborate aryldiazonium salts, employing $\left[\mathrm{Ru}(\mathrm{bpy})_{2}\right] \mathrm{Cl}_{2}$ as the photocatalyst and irradiation with a $440 \mathrm{~nm}$ light source in $\mathrm{CH}_{3}-\mathrm{CN} /$ water. 
Scheme 22. Proposed Reaction Mechanism for 1,5Trifluomethylthio-sulfonylation of Vinylcyclopropanes

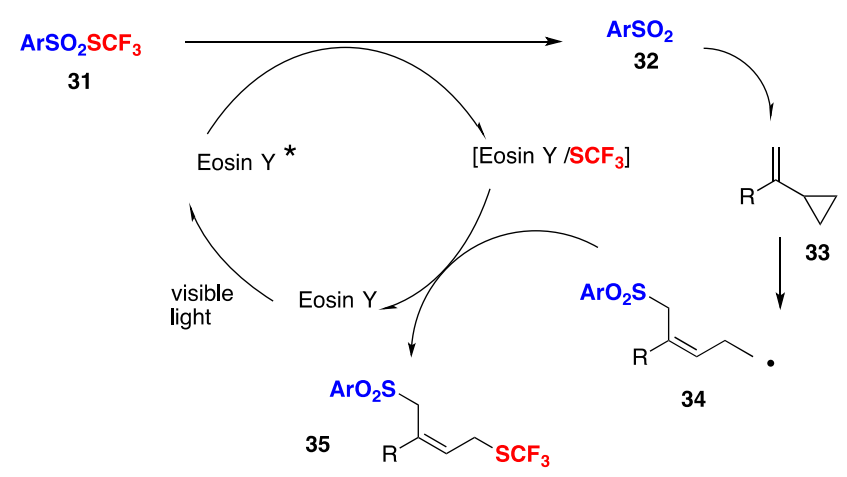

The scope of the transformation regarding the aryl diazonium salt is depicted in Scheme 23.

Scheme 23. Selected Examples for Arylation of Styrene in $\mathrm{MeCN} /$ Water
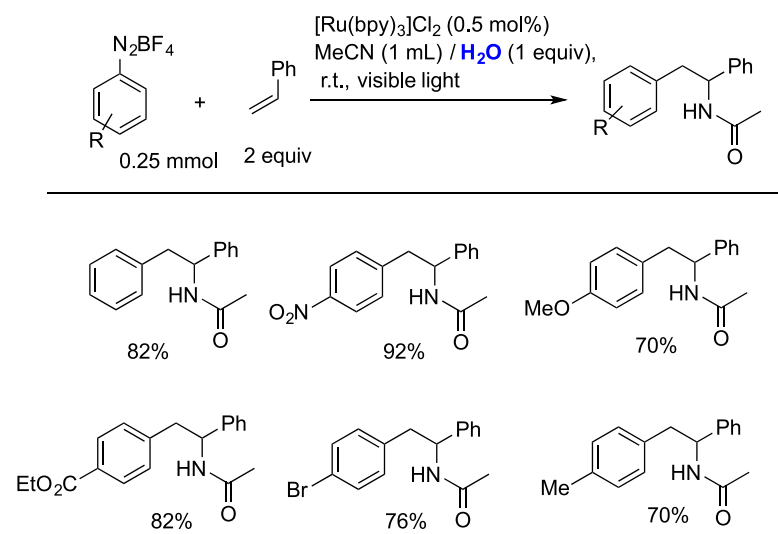

The reaction in terms of the scope of the alkene is illustrated in Scheme 24.

The authors ${ }^{89}$ proposed a mechanism based on trapping experiments and literature precedent, according to Scheme 25.

An aryl radical is formed by an ET from the excited state of the photocatalyst $\left[\mathrm{Ru}(\mathrm{bpy})_{3}\right]^{2+*}$ to diazonium salt and

Scheme 24. Selected Examples for 4-Methoxyphenylation of Alkenes
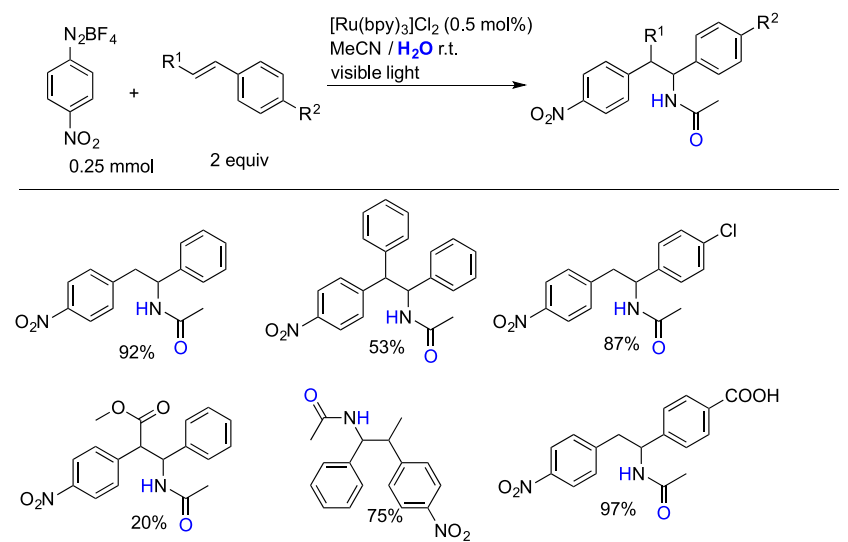

\section{Scheme 25. Proposed Mechanism}

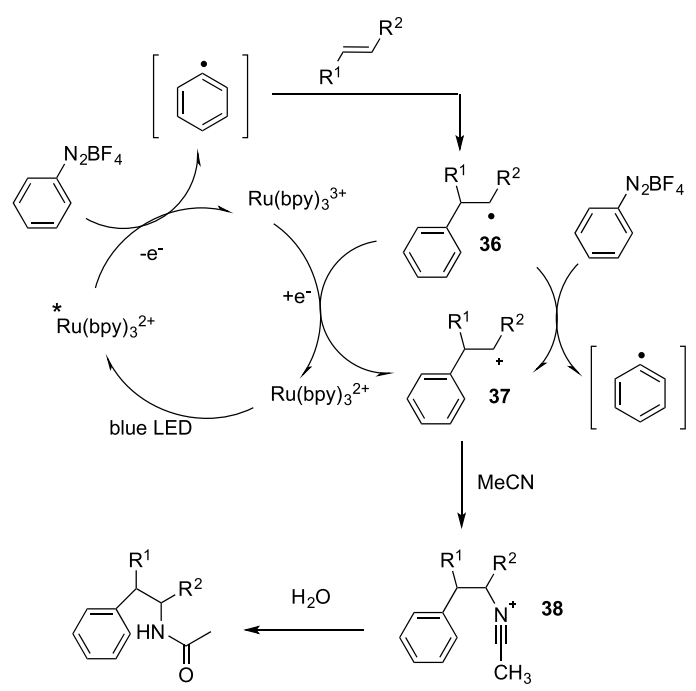

$\mathrm{Ru}(\mathrm{III})$. The addition of an aryl radical to olefin affords radical intermediate 36 , which is then further oxidized to afford carbocation 37 . Finally, intermediate 37 is attacked by the solvent (a nitrile) (intermediate 38 ), followed by hydrolysis to give an amino-arylated product. The participation of water in the reaction is to provide the $\mathrm{H}$ and $\mathrm{O}$ atoms in the water attack of intermediate 38 .

In a more recent report Wang and colleagues ${ }^{90}$ have found an effective and benign visible light-induced Meerwein hydratation reaction of alkenes in water.

The reaction employs diazonium salts bearing various electron-poor substituents on the aryl ring as aryl radical sources, an alkene as the radical acceptor, and mpg- $\mathrm{C}_{3} \mathrm{~N}_{4}$ as the photocatalyst in water as the solvent under Xe light irradiation.

As shown in Scheme 26, a wide array of aryl diazonium salts and alkenes were tested. The reaction had good tolerance with several groups, such as nitro, acyl, cyanide, ester, methyl, and halogen groups giving the corresponding products in good yields.

Scheme 26. Aryldiazonium Salts and Alkene Scope: Photocatalytic Meerwein Hydratation Reaction

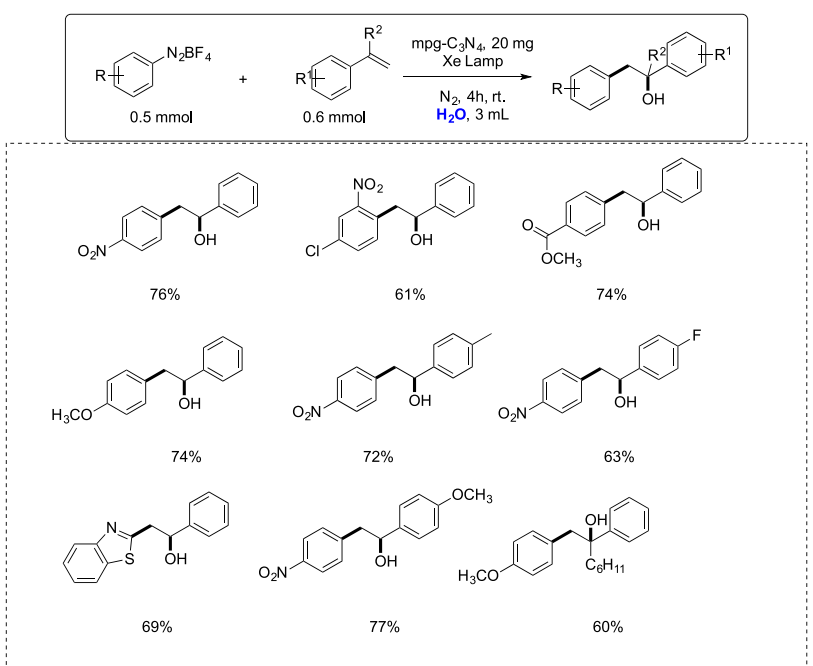


Remarkably, the reaction could be driven by sunlight with good results compared with the model with Xe light irradiation (Scheme 27). These results indicated the suitability of the protocol for large-scale applications. ${ }^{90}$

Scheme 27. Photocatalytic Meerwein Hydratation Reaction Promoted by Sunlight

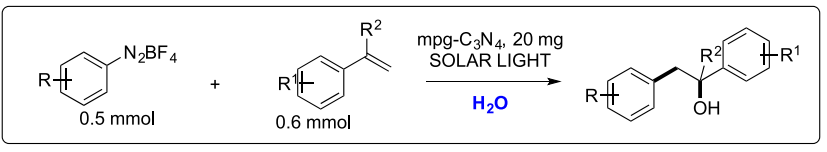<smiles>N#Cc1ccc(CC(O)c2ccccc2)cc1</smiles>

$76 \%$

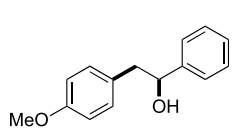

$76 \%$

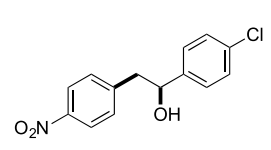

$78 \%$

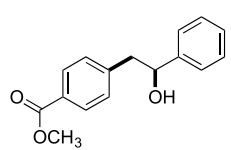

$78 \%$
$75 \%$

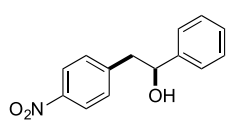

In the mechanistic proposal shown in Scheme 28, initially mpg- $\mathrm{C}_{3} \mathrm{~N}_{4}$ is excited upon irradiation; the excited mpg- $\mathrm{C}_{3} \mathrm{~N}_{4}$

Scheme 28. Proposed Mechanism of Photocatalytic Meerwein Hydratation Reaction

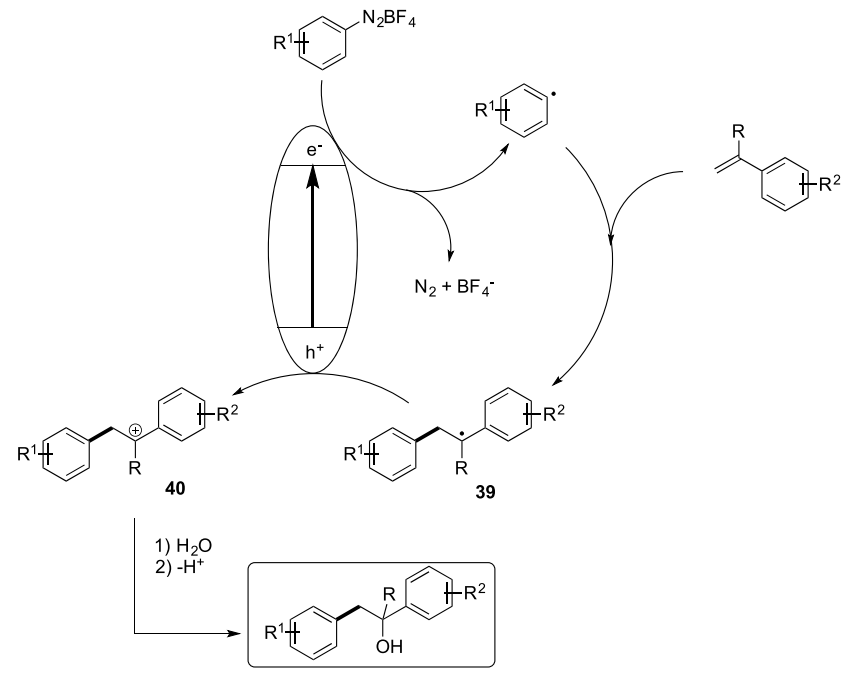

reacts with the aryldiazonium salt in a single electron transfer reaction (SET) to generate the corresponding aryl radical. The aryl radical adds to the alkene to produce benzyl radical 39 (Scheme 28), which is oxidized by the valence band of mpg$\mathrm{C}_{3} \mathrm{~N}_{4}$ to form carbocation 40 (Scheme 28). Finally, $\mathrm{H}_{2} \mathrm{O}$ acts as the nucleophile and reacts with $\mathbf{4 0}$ followed by deprotonation to yield the final product. Although the role of water as the nucleophile is obvious in this process, the feasibility of the reaction in the water heterogeneous system as opposed to in an organic homogeneous mixture is interesting as a protocol alternative.

Chilamari and colleagues ${ }^{91}$ have accomplished the arylation of alkenes starting from arylboronic acids in a mixture of water:methyl acetate ( $\mathrm{pH}$ 7.8) utilizing lumiflavin, a bioinspired flavin photocatalyst, under a nitrogen atmosphere. The scope of the transformation is illustrated in Scheme 29.

During the investigation of the reaction mechanism, the authors ${ }^{91}$ performed a series of experiments, such as quenching of fluorescence of lumiflavin with aryl boronic acid, computational experiments, radical trapping experiments with TEMPO, and deuterium-labeling studies with $\mathrm{D}_{2} \mathrm{O}$. In the mechanism proposed, excited lumiflavin reduces the aryl boronic acid to aryl radicals, which in turn add to the alkene double bond to render an alkyl radical that through a HAT generates the product.

Asymmetric Radical Conjugate Addition to $\alpha, \beta$ Unsaturated Systems. Asymmetric acyl radical conjugate additions toward the synthesis of 1,4-dicarbonyl compounds have very recently been achieved through visible light photocatalysis. $^{92,93}$

Kuang and colleagues ${ }^{94}$ have reported the combination of neutral Eosin $\mathrm{Y}$ with a chiral-at-metal bis-cyclometalated rhodium(III) complex $(\Lambda-\mathrm{RhS}$ or $\Delta-\mathrm{RhS})$. In this manner, aldehydes can be employed as acyl radical precursors to undergo catalytic enantioselective addition to $\alpha, \beta$-unsaturated $N$-acyl-3,5-dimethylpyrazoles with high enantioselectivity.

The authors ${ }^{94}$ found the optimum reaction conditions for the enantioselective reaction consist of neutral Eosin Y ( $1 \mathrm{~mol}$ $\%)$, a chiral-at-metal bis-cyclometalated rhodium(III) complex (4 mol \%), tert-butyl methyl ether (TBME) $(0.1 \mathrm{~mL})$, and water ( 20 equiv) at $10{ }^{\circ} \mathrm{C}$ for 3 days under $18 \mathrm{~W}$ blue LED irradiation. The scope of the reaction is shown in Scheme 30.

As observed from Scheme 30, propionaldehyde, acetaldehyde, 3,3-dimethylbutanal, and benzaldehyde have been used successfully as substrates affording good yields of the asymmetric acyl radical conjugate addition products with high stereoselectivities.

Scheme 29. Representative Examples of Scope of Transformation Regarding Aryl Boronic Acid and Alkene

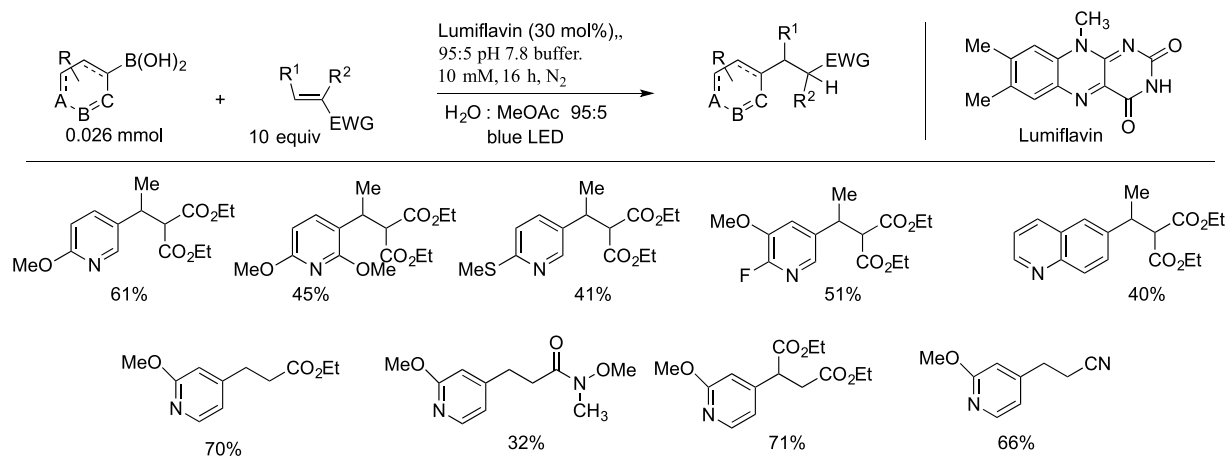


Scheme 30. Aldehyde Scope: Selected Examples of Enantioselective Addition to $\alpha, \beta$-Unsaturated $N$-Acyl-3,5dimethylpyrazoles

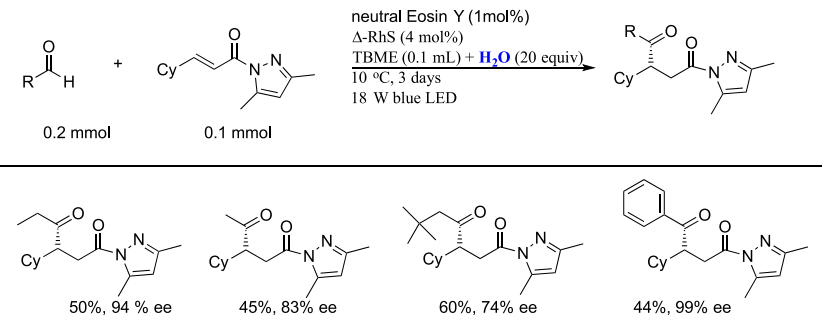

The authors also inspected the reaction in terms of the scope of the unsaturated $\mathrm{N}$-acyl-3,5-dimethylpyrazoles, according to Scheme $31 .^{94}$

Scheme 31. Unsaturated N-Acyl-3,5-dimethylpyrazoles Scope: Selected Examples of Enantioselective Addition to $\boldsymbol{\alpha}, \boldsymbol{\beta}$-Unsaturated $\mathrm{N}$-Acyl-3,5-dimethylpyrazoles
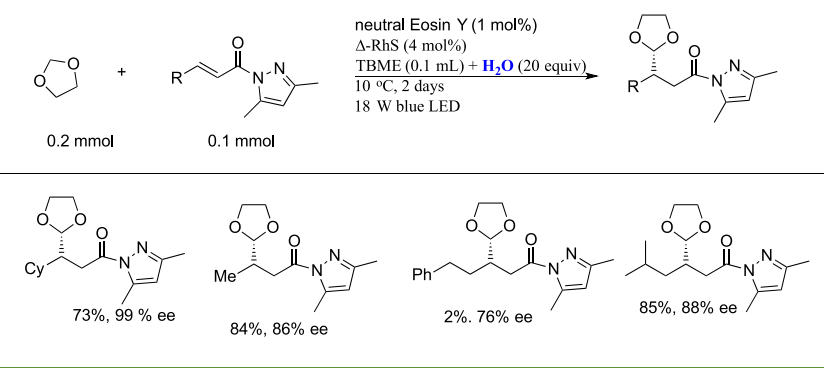

As observed from Scheme 31, $\alpha, \beta$-unsaturated $N$-acyl-3,5dimethylpyrazoles substituted with cyclopentyl, methyl, ethylphenyl, and isopropyl groups afforded high yields and diastereoselectivities of the radical conjugate addition products, employing formaldehyde as the aldehyde source.

The authors ${ }^{94}$ performed a series of control experiments in order to uncover the reaction mechanism. On the basis of redox potentials, excited Eosin $\mathrm{Y}\left(\mathrm{E}\left(\mathrm{S}^{*} / \mathrm{S}^{1-}\right)=+0.83 \mathrm{~V}\right.$ vs
SCE in $\left.\mathrm{CH}_{3} \mathrm{CN} / \mathrm{H}_{2} \mathrm{O}\right)^{95}$ is unable to oxidize an aldehyde to an acyl radical. The reaction is suppressed when TEMPO (a radical scavenger) is used. A plausible reaction mechanism is depicted in Scheme 32.

Acyl radical 41 (Scheme 32) is generated by a HAT reaction between Eosin $\mathrm{Y}^{*}$ and an aldehyde. Radical 41 in turn adds to $\mathrm{N}-\mathrm{O}-$ rhodium-coordinated $\mathrm{N}$-acyl pyrazole substrate 42 to produce radical intermediate $\mathbf{4 3}$ that suffers reverse HAT with Eosin $\mathrm{Y}-\mathrm{H}$. Ligand exchange between intermediate $\mathbf{4 4}$ and the starting unsaturated amide delivers the chiral product and regenerates the active complex (Scheme 32)..$^{4}$

\section{DECARBOXYLATIVE AND DEOXYGENATIVE REACTIONS IN AQUEOUS MEDIA}

Photoinduced decarboxylative functionalization has been employed to build an impressive array of functional molecules, ${ }^{96-99}$ such as decarboxylative conjugate addition, ${ }^{100,101}$ fluorination, ${ }^{102,103}$ and borylation. ${ }^{104,105}$ Photocatalysis has recently played a relevant role in decarboxylative reactions. There are recent review articles on single electron activation of arylcarboxylic acids ${ }^{106-108}$ where carboxylate groups can be transformed into reactive aryl carboxylic radicals, aryl radicals, and acyl radicals by electrocatalysis, photocatalysis, or in the presence of some SET oxidants. ${ }^{106}$ Also, the deoxygenative functionalization of alcohols and carboxylic acids is an appealing approach for the conversion of these feedstocks into high-value products. ${ }^{109}$ However, the direct deoxygenative transformation under mild conditions is cumbersome due to the lack of proper methods for the activation of the strong $\mathrm{C}-\mathrm{O}$ bonds $\left(102 \mathrm{kcal} \mathrm{mol}^{-1}\right)$. More recently, the strategy of visible light photocatalytic phosphoranyl radical fragmentation affords a novel entry for $\mathrm{C}-\mathrm{O}, \mathrm{N}-$ $\mathrm{O}$, and $\mathrm{S}=\mathrm{O}$ bond activation. ${ }^{110}$ Photocatalytic strategies for deoxygenation of alcohols and carboxylic acids through phosphoranyl radical fragmentation have recently been reviewed. ${ }^{109,111}$

$\mathrm{Wu}$ and collaborators ${ }^{112}$ developed a one-pot cascade process combining a photocatalyst with ketoreductase

\section{Scheme 32. Proposed Reaction Mechanism}

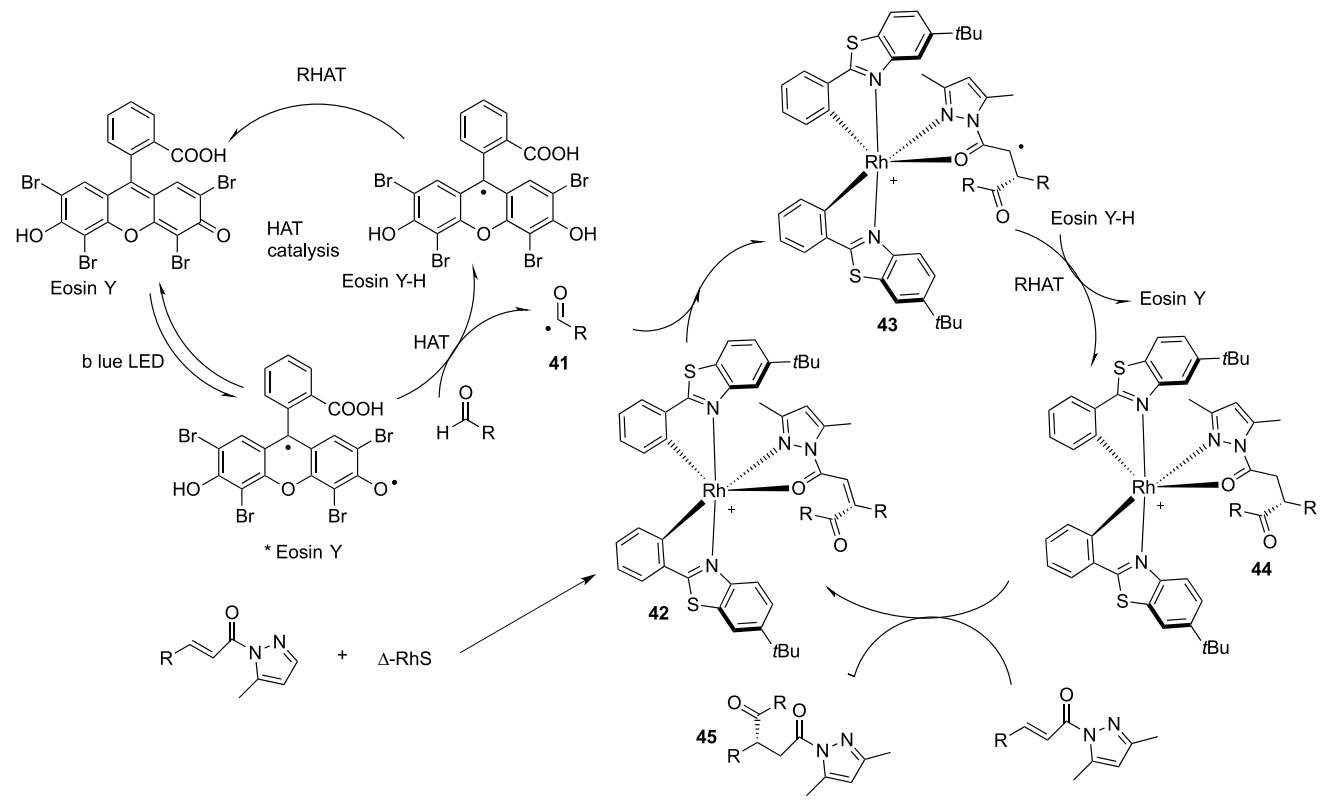


(KRED) to convert carboxylic acids into chiral alcohols in water (Scheme 33).

Scheme 33. Combining Photocatalysis and Biocatalysis: Summary of Reaction Pathway

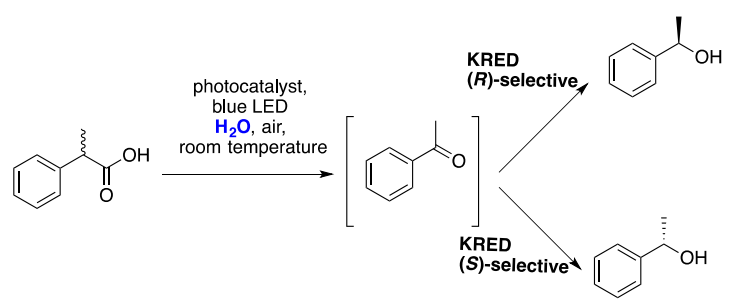

For the formation of the keto product, the authors ${ }^{112}$ found optimized reaction conditions involving 2-phenylpropionic acid $(0.06 \mathrm{mmol})$ as the substrate, 2-sodium anthraquinone sulfonate, SAS ( $5 \mathrm{~mol} \%$ ) as the photocatalyst, and water as the solvent $(2 \mathrm{~mL})$ under blue LED irradiation. Under these reaction conditions, a $95 \%$ yield of acetophenone is obtained.

The authors ${ }^{112}$ investigated the scope of the reaction under the optimized conditions considering different arylcarboxylic acids, according to Scheme 34.

Scheme 34. Selected Examples for Decarboxylation, Followed by Capturing $\mathrm{O}_{2}$ and Then Conversion into Corresponding Ketones

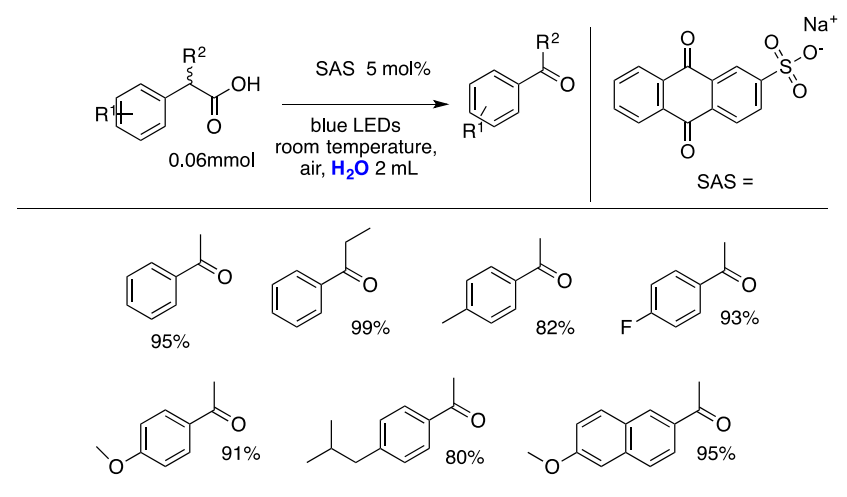

A scale-up of the reaction $(6.7 \mathrm{mmol}$ of phenylpropionic acid) was carried out, and product acetophenone was obtained in a satisfactory yield (92\%) after $30 \mathrm{~h}$ of irradiation. ${ }^{112}$ The authors continued with the improvement of the one-pot protocol, thus employing a ketone reductase (KRED) for catalyzing the asymmetric reduction of the ketones. The selected enzyme was a carbonyl reductase from Kluyveromyces thermotolerans (KtCR) as a model enzyme, obtaining high enantioselectivities for the reduced alcohols, as shown in Scheme $35 .^{112}$

For the $S$-selective KRED, the authors employed YueD from Bacillus sp. ECU0013 $3^{113,114}$ which provided mainly $(S)$ alcohols through the above one-pot cascade reaction in very good enantiomeric excess and yields.

Although the authors did not discuss the mechanism involved, ${ }^{00}$ a likely reaction pathway would entail excitation of an anthraquinone-2-sufonic acid photocatalyst, whose triplet excited state would oxidize the carboxylic acid to its radical cation. The radical cation of the carboxylic acid would readily deprotonate affording the carboxylate radical which would undergo decarboxylation to yield a secondary benzyl radical.
Scheme 35. Substrate Scope of (R)-Selective One-Pot Decarboxylative Hydroxylation

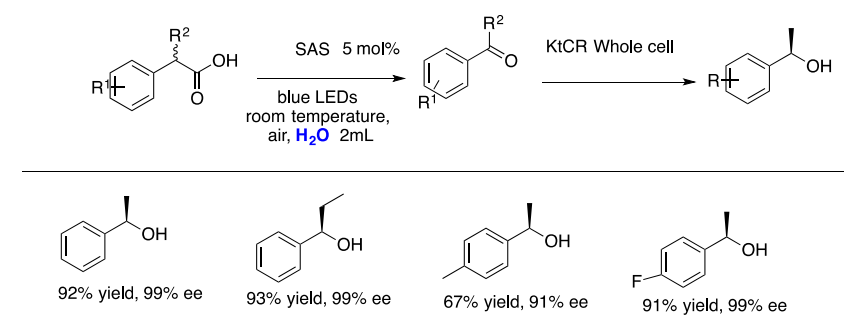

This secondary benzyl radical would probably react with oxygen $^{115}$ to afford to a ketone product; however, this last step of the reaction is conjectural and needs to be explored by further experimental evidence.

Zhang and colleagues ${ }^{116}$ have recently reported a visible light-mediated photocatalytic deoxygenation of aryl carboxylic acids through the employment of $\mathrm{PPh}_{3}$ which triggers the deoxygenative carbon-carbon coupling of aryl-carboxylic acids with olefins in an aqueous phase leading to aromatic ketones. The authors ${ }^{116}$ found that the best reaction conditions employ aryl carboxylic acid ( $0.2 \mathrm{mmol}, 1$ equiv), alkene (1.5 equiv), photocatalyst $\operatorname{Ir}\left[\mathrm{dF}\left(\mathrm{CF}_{3}\right) \mathrm{ppy}\right]_{2}(\mathrm{dtbby}) \mathrm{PF}_{6}(1 \mathrm{~mol} \%), \mathrm{K}_{2}$ $\mathrm{HPO}_{4}(20 \mathrm{~mol} \%)$, and $\mathrm{PPh}_{3}$ (1.2 equiv). The authors investigated the scope of the reaction in terms of the arylcarboxylic acid and the alkene. A summary of the scope achieved for both aryl carboxylic acid and alkene is shown in Scheme 36.

The authors further applied the strategy with the aromatic moieties of known drugs such as telmisartan (specific antagonist of angiotensin II receptor), hiestrone (antineoplastic agent), and adapalene (for the treatment of acne), which readily suffered deoxygenative ketone synthesis.

In order to explore the mechanism, the authors ${ }^{116}$ carried out some control experiments, such as the reaction with 2,2,6,6-tetramethylpiperidin-1-yl)oxyl (TEMPO) and 2,6-ditert-butyl-p-cresol. These additives inhibited the reaction completely. In the case of TEMPO, the adduct between the acyl radical and TEMPO was found, which purports to the presence of an acyl radical intermediate. When the authors ${ }^{116}$ employed 2-allylbenzoic acid as the substrate, the cyclization product 2-methyl-2,3-dihydro-1H-inden-1-one was obtained (Figure 1a), indicating the presence of acyl radicals. Deuterium-labeling experiments in $\mathrm{D}_{2} \mathrm{O}$ showed that water was the source of the proton. When $\mathrm{H}_{2}{ }^{18} \mathrm{O}$ was used as the solvent, the aromatic acid labeled with ${ }^{18} \mathrm{O}$ (Figure 1b) indicated that the oxygen atom in $\mathrm{Ph}_{3} \mathrm{P}=\mathrm{O}$ arrives from the carboxylic acid and not from water. When the potassium salt of benzoic acid was used instead of free benzoic acid, a deoxygenative coupling product was obtained in moderate yield, which implied that the $\mathrm{Ph}_{3} \mathrm{P}^{\bullet+}$ radical cation reacts with benzoate (Figure 1c). Stern Volmer experiments revealed that the excited state of the photocatalyst (i.e., $\operatorname{Ir}\left[\mathrm{dF}\left(\mathrm{CF}_{3}\right)\right.$ ppy $]_{2}$ (dtbby) $\mathrm{PF}_{6} *$ ) is suppressed by $\mathrm{Ph}_{3} \mathrm{P}$.

A possible reaction mechanism is depicted in Scheme 37. Visible light excitation of the photocatalyst (i.e., $\operatorname{Ir}\left[\mathrm{dF}\left(\mathrm{CF}_{3}\right)\right.$ ppy $\left.]_{2}(\mathrm{dtbby}) \mathrm{PF}_{6}\right)$ leads to a triplet charge transfer state (metal-to-ligand) which oxidizes $\mathrm{Ph}_{3} \mathrm{P}$ to its radical cation and the photocatalyst resulting in its reduced redox manifold. $\mathrm{Ph}_{3} \mathrm{P}^{\bullet+}$ species combines with the aryl carboxylate ion to form a $\mathrm{P}$-centered radical, provoking the scission of the $\mathrm{O}-\mathrm{C}=\mathrm{O}$ bond, resulting in an acyl radical and $\mathrm{Ph}_{3} \mathrm{P}=\mathrm{O}$. Acyl radical 
Scheme 36. Scope of Deoxygenative Carbon-Carbon Coupling of Aryl-Carboxylic Acids with Olefins in Aqueous Phase Leading to Aromatic Ketones
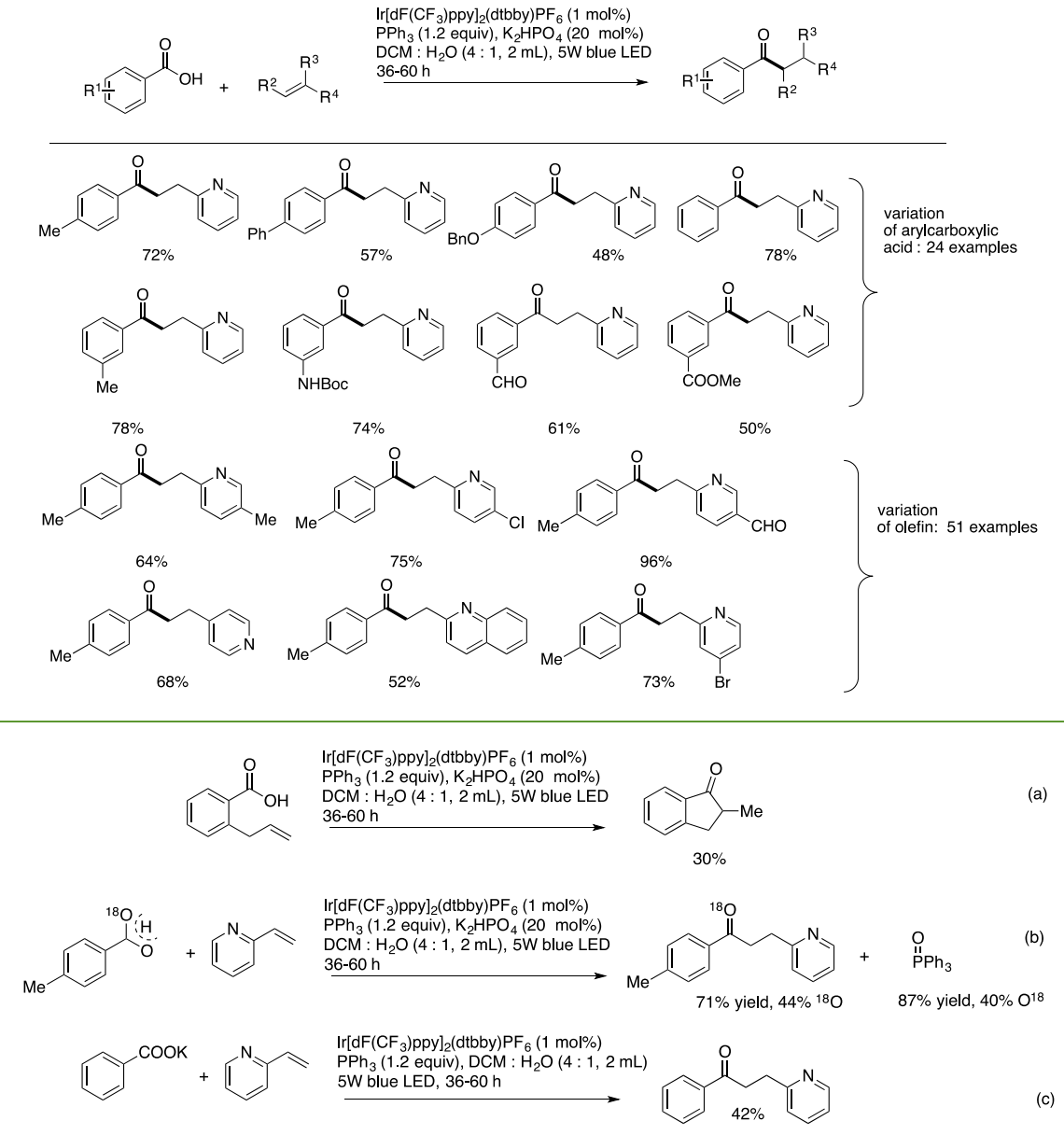

Figure 1. Mechanistic probe experiments.

Scheme 37. Proposed Reaction Mechanism

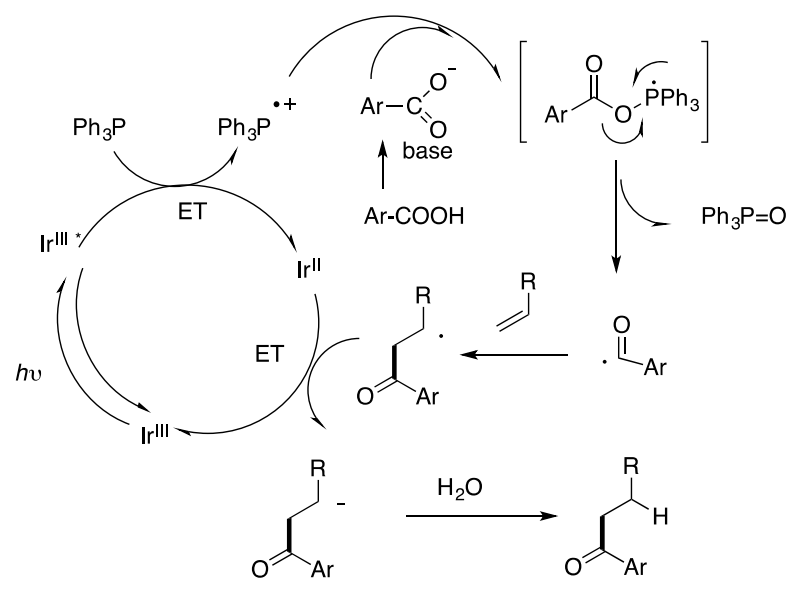

attacks the double bond from the olefin giving a radical adduct which is further reduced by the lower oxidation state of the photocatalyst (i.e., $\operatorname{Ir}(\mathrm{II})$ ) to yield a carbanion, which upon protonation from water affords the final product (Schgem 35c).

He and collaborators ${ }^{117}$ presented a visible light-induced deoxygenative $\mathrm{C} 2$-sulfonation of quinoline $\mathrm{N}$-oxides in aqueous media. Optimized reaction conditions were achieved employing quinoline $\mathrm{N}$-oxide as the model substrate, $\mathrm{Na}_{2}$. Eosin $\mathrm{Y}$ as the photocatalyst, and 4-methylbenzenesulfinic acid as the sulfonating reagent in acetone: $\mathrm{H}_{2} \mathrm{O}(1.5: 1)$ as the reaction medium with irradiation with blue LEDs under an air atmosphere (Scheme 38). ${ }^{117}$ The reaction is successfully

Scheme 38. Selected Examples for Visible Light-Induced Deoxygenative C2-Sulfonation of Quinoline $\mathrm{N}$-Oxides with Sulfinic Acids in Aqueous Media

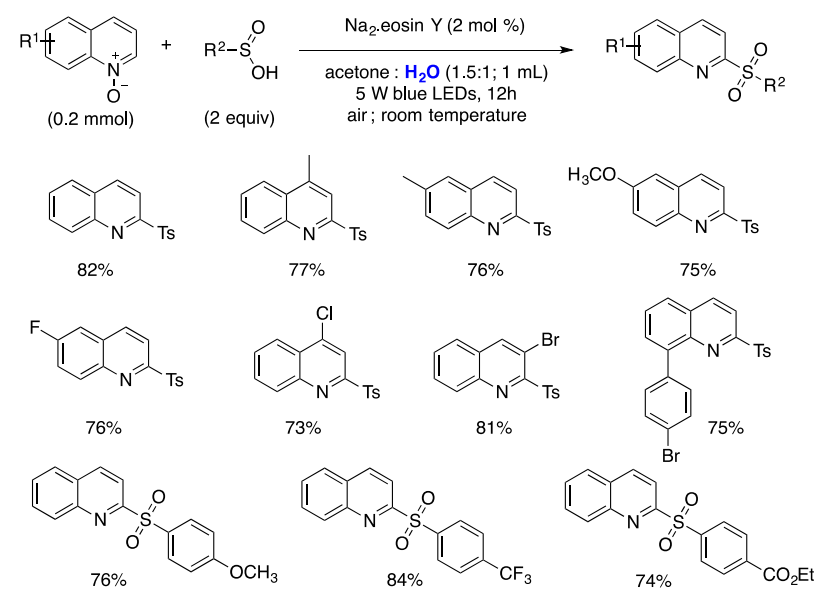


applied to different substituted quinoline $N$-oxide substrates with electron-withdrawing and electron-donating groups including halogen atoms. Regarding the sulfinic acid reagent, different benzenesulfonic acids bearing electron-rich and electron-poor substituents, such as ester, halides, trifluoromethyl, phenyl, and methoxy, all underwent the reaction and afforded their respective products in very good yields (Scheme 38). ${ }^{117}$

The authors performed a series of experiments including radical trapping with TEMPO, electron paramagnetic resonance studies, redox potential measurements, and kinetic isotope effect experiments in order to clarify the reaction mechanism. On the basis of all the evidence collected, the authors proposed a reaction mechanism (Scheme 39) where

Scheme 39. Proposed Reaction Mechanism for Visible Light-Induced Deoxygenative C2-Sulfonation of Quinoline $\mathrm{N}$-Oxides with Sulfinic Acids in Aqueous Media

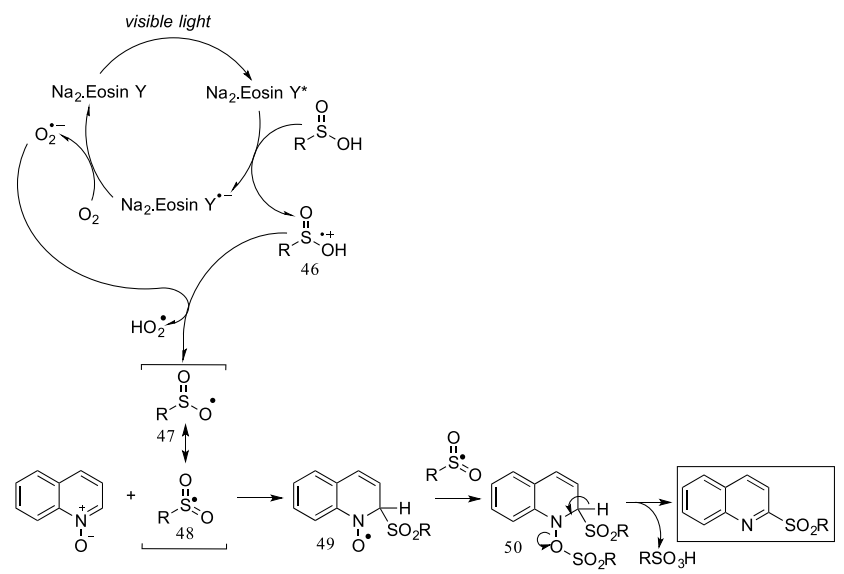

$\mathrm{Na}_{2} \cdot$ Eosin $\mathrm{Y}$ upon excitation with visible light performs a single electron oxidation of the sulfinic acid reagent, yielding sulfonyl radical cation 46 (Scheme 39) and the $\mathrm{Na}_{2}$.Eosin $\mathrm{Y}^{\circ-}$ radical anion which is further oxidized by $\mathrm{O}_{2}$ present in air to afford $\mathrm{Na}_{2}$. Eosin $\mathrm{Y}$ in the ground state and anion superoxide $\mathrm{O}_{2}{ }^{\bullet-}$. Radical cation 46 (Scheme 39) undergoes deprotonation by $\mathrm{O}_{2}{ }^{\bullet-}$ providing $\mathrm{HO}_{2} \bullet$ and radical intermediate 47 (Scheme 39) which is in resonance with sulfur-centered radical intermediate 48 (Scheme 39). The quinoline $\mathrm{N}$-oxide substrate reacts with radical intermediate 48 (Scheme 39) producing radical intermediate 49 (Scheme 39), which furthers combines with a second sulfur-centered radical intermediate $\mathbf{4 8}$ (Scheme 39) to yield intermediate 50 (Scheme 39), which undergoes a dehydro-aromatization process delivering the 2-sulfonylquinoline reaction product and releasing a sulfonic acid molecule. ${ }^{117}$

A transition metal-free photocatalytic methodology for the decarboxylative fluorination of aliphatic carboxylic acids employing a mixture of acetonitrile and water (1:1) as the reaction medium was reported by Ye and collaborators. ${ }^{103}$ The most effective conditions for this transformation are met when employing 9-mesityl-10-methyl-acridinium perchlorate (Mes$\mathrm{AcrClO}_{4}$ ) or riboflavin as photocatalysts, $\mathrm{Cs}_{2} \mathrm{CO}_{3}$ as the base, Selectfluor as the fluorine source, and irradiation with a commercial fluorescent lamp (CFL) at room temperature and under a $\mathrm{N}_{2}$ atmosphere (Scheme 40). ${ }^{103}$ The reaction worked successfully with primary, secondary, and tertiary carboxylic acids, including $\mathrm{N}$-protected amino acids with phthaloyl or succinyl groups (Scheme 40). ${ }^{103}$
Scheme 40. Selected Examples for Decarboxylative Fluorination of Aliphatic Carboxylic Acids by Photoredox Catalysis Employing a Mixture of $\mathrm{MeCN}: \mathrm{H}_{2} \mathrm{O}(1: 1)$ as Reaction Media

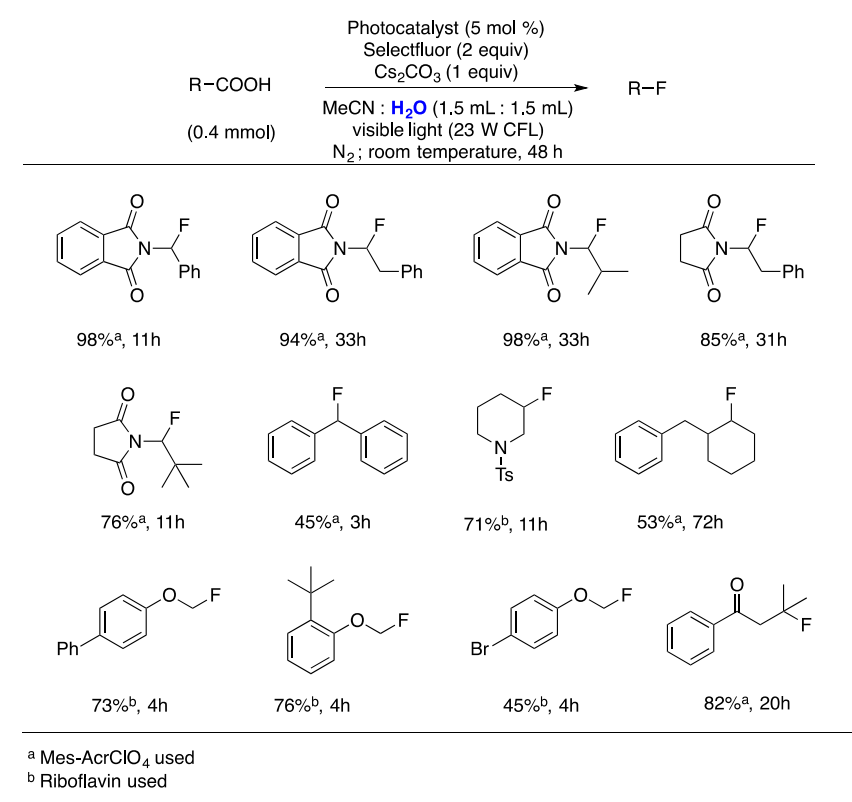

The authors ${ }^{103}$ proposed a reaction mechanism based on experimental evidence, including luminescence suppression experiments, (Scheme 41) where the excited state of the

Scheme 41. Proposed Reaction Mechanism for Decarboxylative Fluorination of Aliphatic Carboxylic Acids by Photoredox Catalysis Employing a Mixture of MeCN: $\mathrm{H}_{2} \mathrm{O}(1: 1)$ as Reaction Media

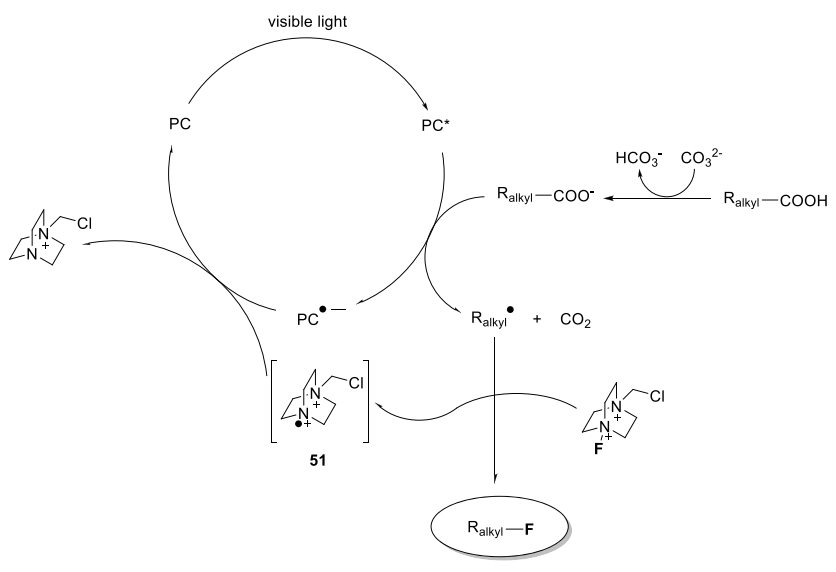

photocatalyst performs a single electron oxidation of the alkyl carboxylate yielding an alkyl radical, $\mathrm{CO}_{2}$, and the radical anion of the photocatalyst. Then, a fluorine atom is transferred from Selectfluor to the alkyl radical yielding the reaction product and radical dication 51 (Scheme 41). The authors proposed the closing of the photoredox cycle by a single electron transfer process from the photocatalyst radical anion to radical dication 51, regenerating the photocatalyst (Scheme 41). ${ }^{103}$

Macmillan and collaborators ${ }^{102}$ presented a protocol for the photocatalyzed decarboxylative fluorination of aliphatic acids in aqueous media. The optimized reaction conditions consist of the reactions of carboxylic acids with Selectfluor in the presence of the heteroleptic $\operatorname{Ir}(\mathrm{III})$ photocatalyst $\operatorname{Ir}\left[\mathrm{dF}\left(\mathrm{CF}_{3}\right)\right.$ - 
ppy $]_{2}$ (dtbbpy) $\mathrm{PF}_{6}$ with of $\mathrm{Na}_{2} \mathrm{HPO}_{4}$ as the base in $\mathrm{CH}_{3}$ $\mathrm{CN}$ :water (1:1) as the reaction medium and irradiation with blue LEDs under an Ar atmosphere. The scope of the reaction is extended to a broad family of alkyl carboxylic acids including primary, secondary, tertiary, benzylic, and homobenzylic carboxylic acids. Substrates bearing heteroatoms in the $\alpha$ or $\beta$ position of the carboxylic group also gave the corresponding reaction products in high yields (Scheme 42$).{ }^{102}$

Scheme 42. Selected Examples for Photocatalyzed

Decarboxylative Fluorination of Aliphatic Acids Employing Selectfluor in the Presence of $\operatorname{Ir}\left[\mathrm{dF}\left(\mathrm{CF}_{3}\right) \mathrm{ppy}\right]_{2}(\mathrm{dtbbpy}) \mathrm{PF}_{6}$ with of $\mathrm{Na}_{2} \mathrm{HPO}_{4}$ as Base in $\mathrm{CH}_{3} \mathrm{CN}$ :water (1:1) as Reaction Media

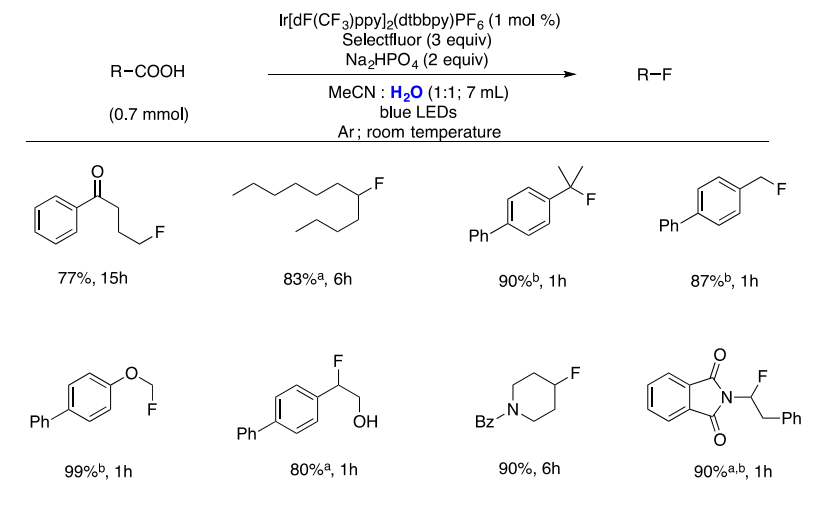

abtained as racemate

reaction run using 2 equiv of Selectluor

The authors ${ }^{102}$ proposed a reaction mechanism (Scheme 43) starting with the photoexcitation of the photocatalyst

Scheme 43. Proposed Reaction Mechanism for Photocatalyzed Decarboxylative Fluorination of Aliphatic Acids Employing Selectfluor in the Presence of $\operatorname{Ir}\left[\mathrm{dF}\left(\mathrm{CF}_{3}\right) \text { ppy }\right]_{2}(\mathrm{dtbbpy}) \mathrm{PF}_{6}$ with of $\mathrm{Na}_{2} \mathrm{HPO}_{4}$ as Base in $\mathrm{CH}_{3} \mathrm{CN}$ :water (1:1) as Reaction Media

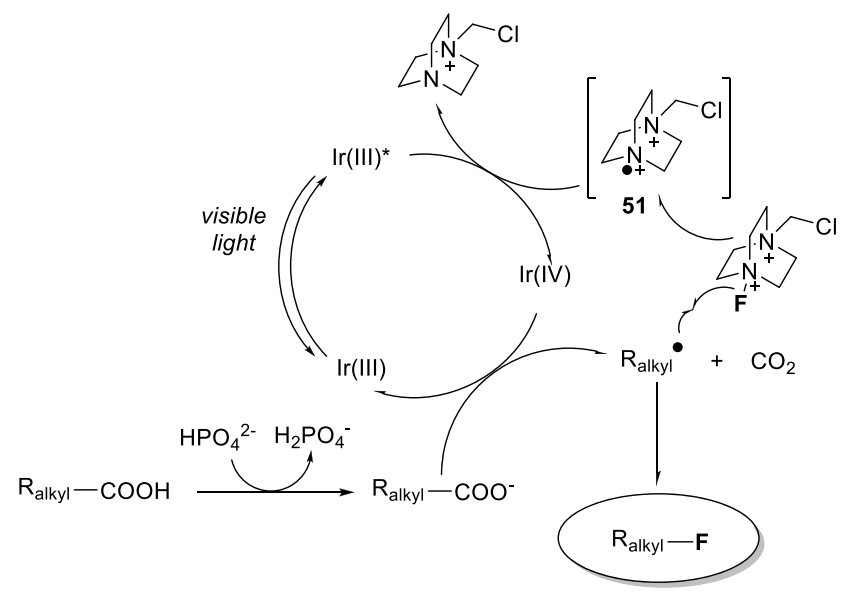

$\operatorname{Ir}\left[\mathrm{dF}\left(\mathrm{CF}_{3}\right) \text { ppy }\right]_{2}$ (dtbbpy) $\mathrm{PF}_{6}[\operatorname{Ir}(\mathrm{III})]$ to afford $* \operatorname{Ir}\left[\mathrm{dF}\left(\mathrm{CF}_{3}\right)-\right.$ ppy $]_{2}(\mathrm{dtbbpy})^{+}\left[\operatorname{Ir}(\mathrm{III})^{*}\right]$. It is important to note that the authors' proposal ${ }^{102}$ includes an initial reduction of a sacrificial quantity of Selectfluor for obtaining $\operatorname{Ir}\left[\mathrm{dF}\left(\mathrm{CF}_{3}\right)\right.$ ppy $]_{2}(\mathrm{dtbbpy})^{2+}[\operatorname{Ir}(\mathrm{IV})]$, and this reaction is not included in Scheme 43. The carboxylic acid is deprotonated by $\mathrm{HPO}_{4}{ }^{2-}$ and affords the alkyl carboxylate that performs a single electron transfer process with $\operatorname{Ir}\left[\mathrm{dF}\left(\mathrm{CF}_{3}\right) \text { ppy }\right]_{2}(\mathrm{dtbbpy})^{2+}[\operatorname{Ir}(\mathrm{IV})]$ regenerating the $\operatorname{Ir}(\mathrm{III})$ photocatalyst and yielding an alkyl radical and $\mathrm{CO}_{2}$. The alkyl radical abstracts a fluorine atom from Selectfluor giving the reaction product and radical cation intermediate $\mathbf{5 1}$ (Scheme 43) that replaces the initial sacrificial Selectfluor by accepting an electron from Ir(III)* to yield the strongly oxidizing $\operatorname{Ir}(\mathrm{IV})$ that further reacts with another alkyl carboxylate anion propagating the reaction (Scheme 43). ${ }^{102}$

Another example of photoredox catalysis based on a decarboxylative fluorination process was developed Paquin and collaborators, starting from aryloxycarboxylic acids. ${ }^{118}$ Optimal yields were obtained when employing $\mathrm{Ru}(\mathrm{bpy})_{3} \mathrm{Cl}_{2}$ as the photocatalyst, Selectfluor as the fluorine atom source, and $\mathrm{NaOH}$ as the base in either $\mathrm{H}_{2} \mathrm{O}$ or $\mathrm{CH}_{3} \mathrm{CN}: \mathrm{H}_{2} \mathrm{O}$ mixtures as reaction media (depending on carboxylic acid substrate solubility) upon visible light irradiation under a $\mathrm{N}_{2}$ atmosphere (Scheme 44). The reaction scope includes a range of

Scheme 44. Selected Examples for Direct C-F Bond Formation by Means of Photoredox Catalysis Based on a Decarboxylative Fluorination Process Developed by Paquin and Collaborators ${ }^{118}$

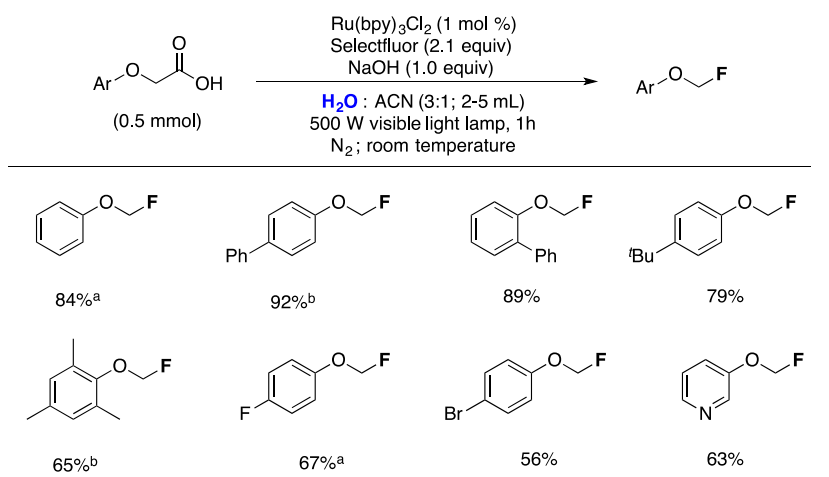

a 3.5 equiv of Selectfluor, 1.5 equiv of $\mathrm{NaOH}, \mathrm{H}_{2} \mathrm{O}$ as solvent

b 3.5 equiv of Selectfluor, 1.5 equiv of $\mathrm{NaOH}, \mathrm{H}_{2} \mathrm{O}: \mathrm{ACN}(1: 1)$ as solvent

aryloxyacetic acid derivatives with the aryloxy ring substituted with alkyl, phenyl, fluorine, or bromine substituents; a pyridyl derivative also afforded the fluorinated product in good yield (Scheme 44). ${ }^{118}$

Regarding some mechanistic aspects, the authors ${ }^{118}$ proved, by transient absorption (TA) spectroscopy experiments, that the reaction proceeds through a photoredox pathway involving a single electron transfer process from the triple metal-toligand charge transfer state $\left({ }^{3} \mathrm{MLCT}\right)$ of the $\mathrm{Ru}(\mathrm{bpy})_{3}{ }^{2+}$ catalyst to Selectfluor forming $\mathrm{Ru}(\mathrm{bpy})_{3}{ }^{3+}$; the last being the key oxidant of the reaction that enables ulterior decarboxylative fluorination. ${ }^{118}$ In this sense, TA spectroscopy of $\mathrm{Ru}(\mathrm{bpy})_{3}\left(\mathrm{PF}_{6}\right)_{2}$ matches previous reports, ${ }^{119}$ with a depletion of the band at $450 \mathrm{~nm}$ corresponding to $\mathrm{Ru}(\mathrm{bpy})_{3}{ }^{2+}$ and an increase of the band at $375 \mathrm{~nm}$, which is assigned to $3 *\left[\mathrm{Ru}(\mathrm{bpy})_{2}\left(\mathrm{bpy}^{\bullet-}\right)\right]^{2+}$. The spectra difference suggests that there is no interaction between the catalyst and phenoxyacetic acid. However, the excited state difference the spectrum of $\mathrm{Ru}(\mathrm{bpy})_{3}\left(\mathrm{PF}_{6}\right)_{2}$ in the presence of an excess of Selectfluor demonstrates the growth of a new absorption band centered at $450 \mathrm{~nm}$. These findings are consistent with an ET pathway between ( $\left.{ }^{3} \mathrm{MLCT}\right)$ and Selectfluor, affording oxidized Selectfluor (radical cation) and $\mathrm{Ru}(\text { bpy })_{3}{ }^{3+}$, which oxidizes aryloxyacetic acid (Figure 2). 


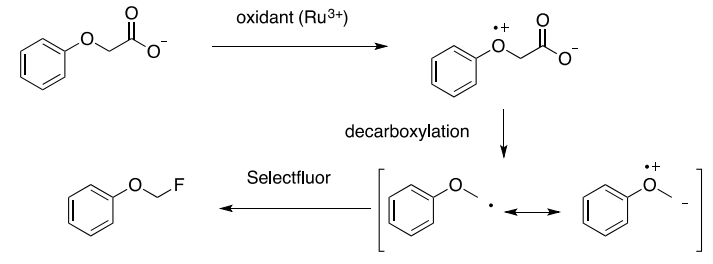

Figure 2. Oxidation and decarboxylation of aryloxyacetic acids.

A photocatalytic decarboxylative/defluorinative $[4+3]$ annulation of $o$-hydroxyphenylacetic acids and trifluoromethyl alkenes was reported by Chen and collaborators. ${ }^{120}$ The reaction affords fluorinated dihydrobenzoxepines via two consecutive $\mathrm{C}-\mathrm{F}$ substitutions in a $\mathrm{CF}_{3}$ group. This latter reaction was carried out in DMSO:water as the solvent mixture and employs 1,3,4,5-tetrakis(diphenylamine)-2,6-dicyanobenzene as the photocatalyst.

Zhu and collaborators ${ }^{121}$ achieved a decarboxylative crosscoupling reaction of $\alpha$-oxocarboxylic acids with styrenes enabled by a domino-fluorination-protodefluorination reaction via photoredox catalysis in aqueous media. Optimized reaction conditions were obtained when benzoylformic acid and 4-methylstyrene were allowed to react in the presence of $\operatorname{Ir}\left[\mathrm{dF}\left(\mathrm{CF}_{3}\right) \mathrm{ppy}\right]_{2}$ (dtbbpy) $\mathrm{PF}_{6}$ as the photocatalyst, Selectfluor as the fluorine atom source, and sodium acetate as the base in $\mathrm{CH}_{3} \mathrm{CN}: \mathrm{H}_{2} \mathrm{O}$ (1:1) as the reaction medium, with irradiation with blue LEDs under an Ar atmosphere (Scheme 45). The scope of the $\alpha$-oxocarboxylic acid substrate embraces benzoylformic acid-bearing electron-rich and electron-withdrawing groups including heterocyclic 2-furanyl-, 2-thienyl-, and 2-naphthyl-substituted $\alpha$-oxo acids (Scheme 45). The substrate scope regarding the alkene substrate includes a series of styrenes having substituents such as fluoro, chloro, bromo, methyl, or phenyl groups on the aromatic ring (Scheme 45). ${ }^{121}$

To gain mechanistic insights into the reaction pathway, the authors performed a series of experiments including radical trapping with TEMPO, reaction in the absence of a base, and
Stern-Volmer quenching studies. ${ }^{121}$ On the basis of the experiments performed and on the information available in the literature, the authors proposed a reaction mechanism initiated by photoexcitation of the $\operatorname{Ir}(\mathrm{III})$ photocatalyst to the excited state $\operatorname{Ir}(\mathrm{III}) *$ followed by a reductive quenching process oxidizing the $\alpha$-oxocarboxylic acid affording the reduced $\operatorname{Ir}(\mathrm{II})$ species, acyl radical $\mathbf{5 2}$ (Scheme 46) and $\mathrm{CO}_{2}$. Acyl radical $\mathbf{5 2}$

Scheme 46. Proposed Reaction Mechanism for Decarboxylative Cross-Coupling of $\alpha$-Oxocarboxylic Acids with Styrenes Enabled by Domino-FluorinationProtodefluorination Reaction via Photoredox Catalysis in Aqueous Media

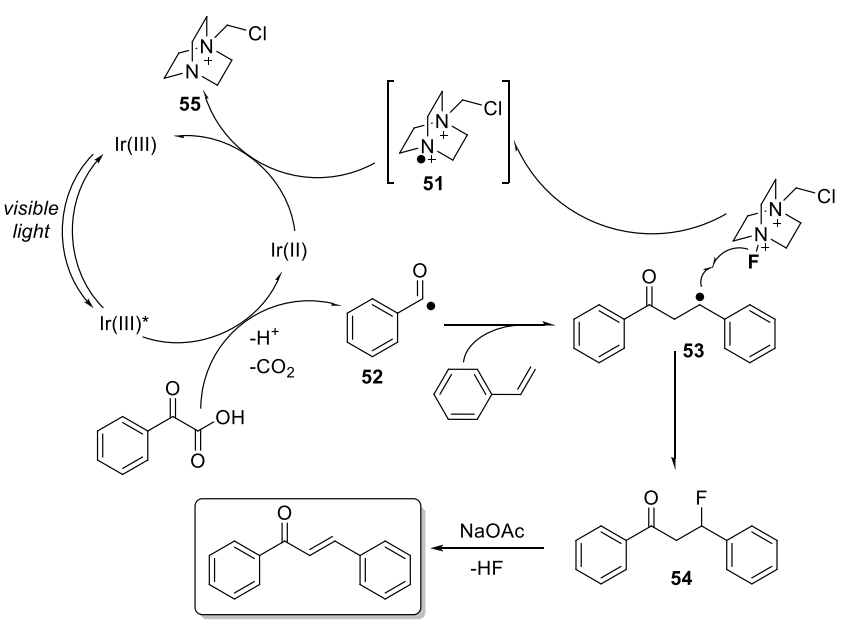

(Scheme 46) adds to the double bond of styrene delivering benzylic radical 53 (Scheme 46) that abstracts a fluorine atom from Selectfluor yielding the acylfluorinated compound 54 (Scheme 46) and Selectfluor radical dication $\mathbf{5 1}$ (Scheme 46) that oxidizes the $\operatorname{Ir}(\mathrm{II})$ catalyst to the ground state $\operatorname{Ir}(\mathrm{III})$ species, thus generating reduced $\mathbf{5 5}$ (Scheme 46). The acylfluorinated compound $\mathbf{5 4}$ (Scheme 46) is unstable and

Scheme 45. Selected Examples for Decarboxylative Cross-Coupling of $\alpha$-Oxocarboxylic Acids with Styrenes Enabled by Domino-Fluorination-Protodefluorination Reaction via Photoredox Catalysis in Aqueous Media

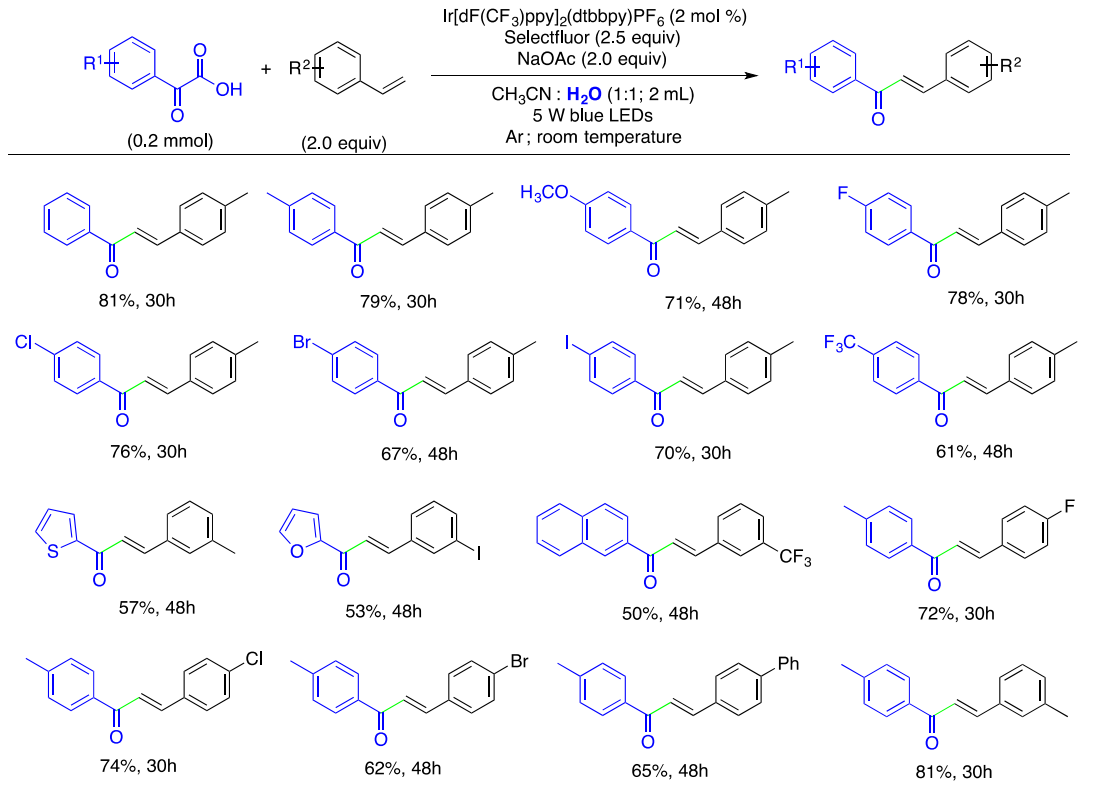


eliminates HF in the presence of a base affording the final $\alpha, \beta$ unsaturated ketone product. ${ }^{121}$

Ramirez and Gonzalez-Gomez ${ }^{122}$ developed a decarboxylative Giese-type reaction of carboxylic acids induced by visible light. The proposed protocol employs 9-mesityl-10methyl-acridinium perchlorate $\left(\mathrm{Mes}-\mathrm{AcrClO}_{4}\right)$ as the photocatalyst, $\mathrm{Na}_{2} \mathrm{CO}_{3}$ as the base, and a mixture of acetonitrile:water $(2: 1)$ as the reaction media, with irradiation under blue LEDs without deoxygenation nor an inert atmosphere (Scheme 47). ${ }^{122}$ The suitable acceptors studied for this

Scheme 47. Selected Examples for Decarboxylative GieseType Reaction of Carboxylic Acids by Photoredox Catalysis Employing Diethyl 2-Ethylidene Malonate as Acceptor and Mixture of $\mathrm{MeCN}: \mathrm{H}_{2} \mathrm{O}(2: 1)$ as Reaction Media

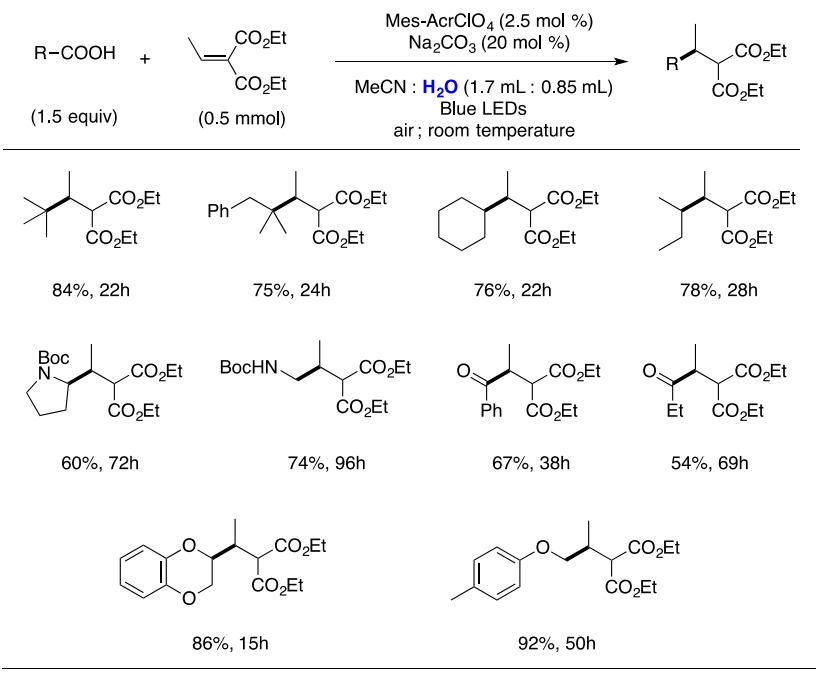

reaction are alkylidene malonates and malonitriles, $\alpha$ substituted acrylates, conjugated ketones and aldehydes, and conjugated sulfones. On the other hand, the scope of carboxylic acids capable of smoothly reacting under these reaction conditions comprises various secondary and tertiary substrates, $\alpha$-amino acids, $\alpha$-keto acids, and $\alpha$-oxy acids. Scheme 47 shows the scope of the reaction when employing a series of suitable carboxylic acids and diethyl 2-ethylidene malonate as the acceptor. ${ }^{122}$
Regarding some mechanistic aspects, the authors, by employing radical scavenger TEMPO, completely inhibited product formation. By performing the reaction using perdeuterated solvents, the authors excluded the probability of $\mathrm{H}$ abstraction of the $\alpha$-acyl radical from the solvent as no deuterated product was observed employing $\mathrm{CD}_{3} \mathrm{CN}: \mathrm{H}_{2} \mathrm{O}$ $(2: 1)$. On the other hand, the single-electron reduction of the $\alpha$-acyl radical formed was verified as a final deuteration product was observed when employing $\mathrm{CH}_{3} \mathrm{CN}: \mathrm{D}_{2} \mathrm{O}(2: 1){ }^{122}$

In 2015, Fu and co-workers ${ }^{123}$ found the decarboxylative 1,4-addition of $\alpha$-oxocarboxylic acids with Michael acceptors by photoredox catalysis. The protocol developed employs an iridium catalyst, $\operatorname{Ir}\left[\mathrm{dF}\left(\mathrm{CF}_{3}\right) \mathrm{ppy}\right]_{2}($ phen $) \mathrm{PF}_{6}$, various 2(hetero)aryl-2-oxocarboxylic acids as acyl radical sources, and different Michael acceptors, $\mathrm{K}_{2} \mathrm{HPO}_{4}$ as the base in a solvent mixture of DCM: $\mathrm{H}_{2} \mathrm{O}(1: 1)$ under $36 \mathrm{~W}$ blue LED irradiation.

Under these reaction conditions, several 2-oxo-2-(hetero)arylacetic acids and a wide range of Michael acceptors, such as $\alpha, \beta$-unsaturated ester, ketone, amide, nitrile, and sulfone, reacted to afford the desired products in good yields (Scheme 48)..$^{123}$

When the reaction of a 2-oxo-2-phenylacetic acid with a Michael acceptor was conducted in the presence of 2.0 equiv of TEMPO, no additional product was observed, and the adduct TEMPO-acyl radical was isolated in high yield as shown in Scheme $49 .^{123}$

Scheme 49. Radical Trapping Experiment with TEMPO

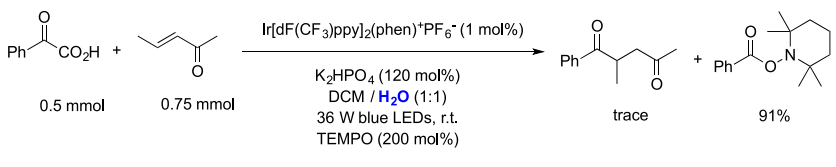

In the hypothesized mechanism (Scheme 50), the photoexcited photocatalyst $\left({ }^{*} \mathrm{M}^{\mathrm{n}}\right)$ can be reductively quenched by $\alpha$ oxocarboxylate to generate an acyl radical, $\mathrm{CO}_{2}$, and the reduced $\mathrm{M}^{\mathrm{n}-1}$. The acyl radical adds to the Michael acceptor to produce radical adduct 56 (Scheme 50) that accepts one electron from $\mathrm{M}^{\mathrm{n}-1}$ to generate $\mathrm{M}^{\mathrm{n}}$ and intermediate 57 (Scheme 50). The 1,4-addition product is formed after protonation of $\mathbf{5 7}$ (Scheme 50$)^{123}$

Scheme 48. Scope: Decarboxylative 1,4-Addition of $\alpha$-Oxocarboxylic Acids with Michael Acceptors

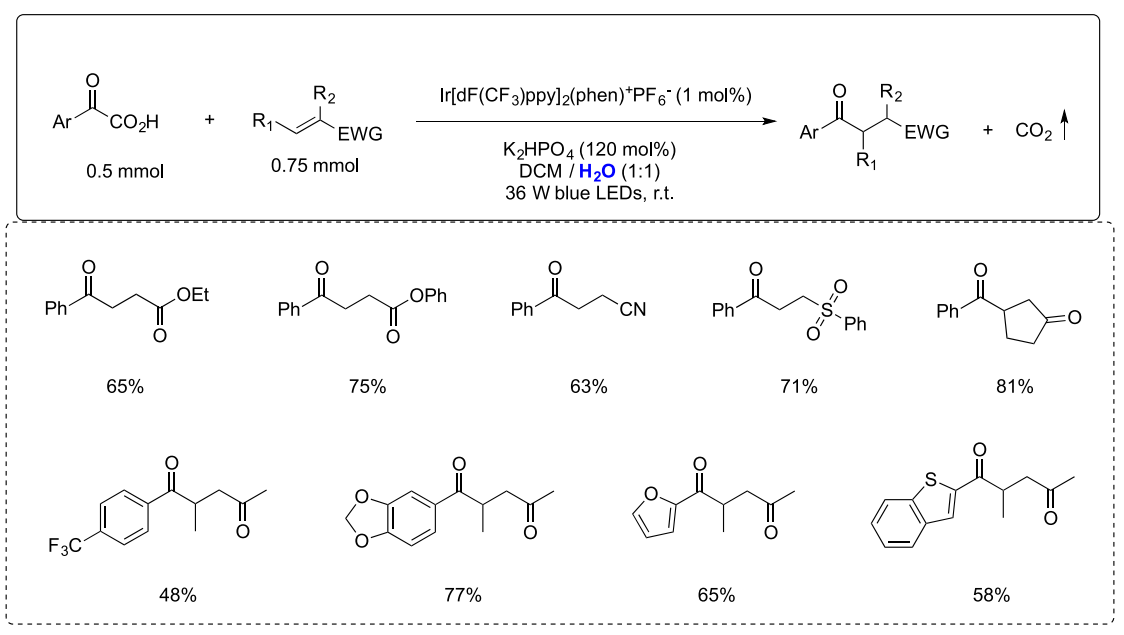


Scheme 50. Proposed Mechanism: Photoredox-Catalyzed Decarboxylative 1,4-Addition of $\alpha$-Oxocarboxylic Acid
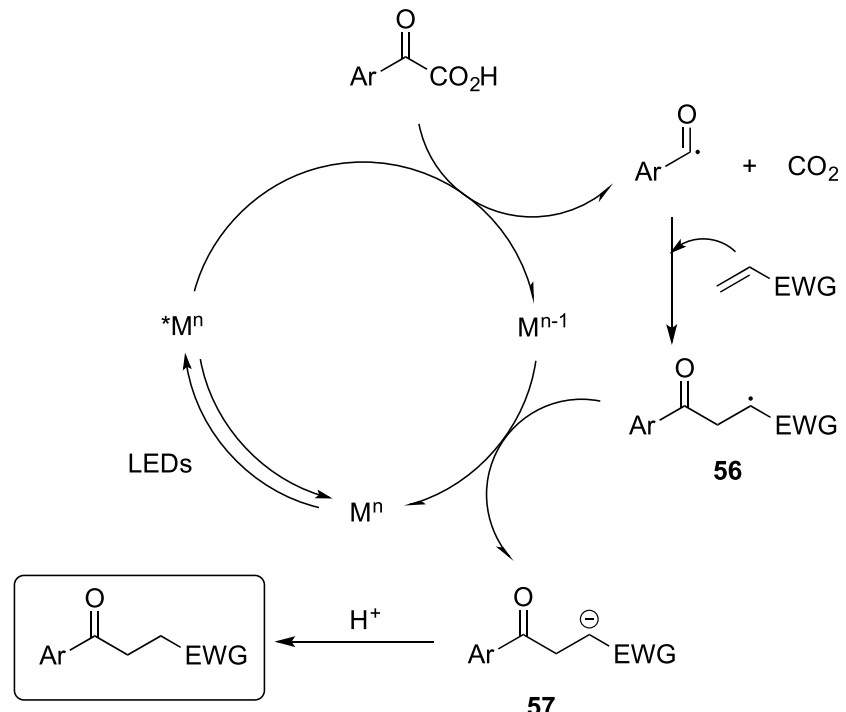

57

A combination of photocatalysis with hypervalent iodine(III) reagents (HIRs) ${ }^{124}$ represents an important tool in the field of iodine chemistry, and numerous organic transformations were achieved in a mild and environmentally benign system, included the aqueous media.

Huang and colleagues ${ }^{125}$ found the deboronative/decarboxylative alkenylation triggered by visible light photoredox catalysis. The optimal reaction conditions (Scheme 51)

Scheme 51. Optimal Reaction Conditions and Scope: Deboronative/Decarboxylative Alkenylation by Photoredox Catalysis

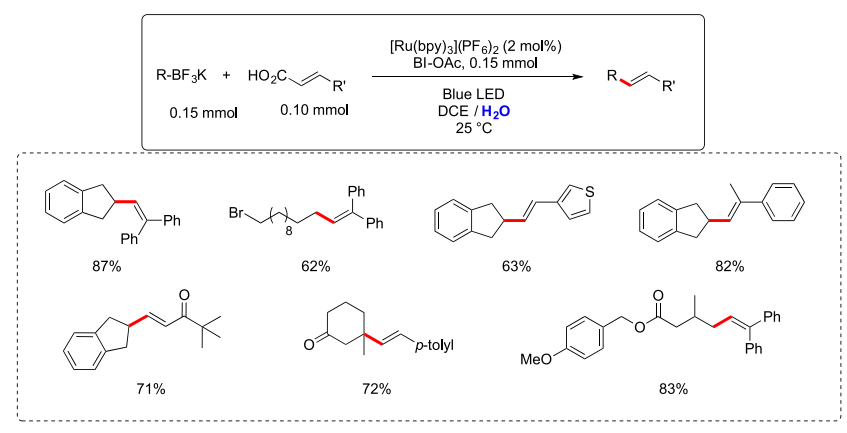

consisted of a mixture of the corresponding alkyl trifluoroboronate and vinyl carboxylic acid, $\left[\mathrm{Ru}(\mathrm{bpy})_{3}\right]\left(\mathrm{PF}_{6}\right)_{2}$ as the photocatalyst, an iodine(III) cyclic regent acethoxybenziodoxole (BI-OAc) as the oxidant in DCE: $\mathrm{H}_{2} \mathrm{O}$ as the solvent mixture under blue LED $\left(\lambda_{\max }=468 \mathrm{~nm}\right)$ irradiation.

As shown in Scheme 51, the system was tested on several substrates, as primary, secondary, and tertiary alkyl trifluoroborates in combination with vinyl carboxylic acids substituted with aryl and acyl groups are rendered in good yields. ${ }^{125}$

To cast some light onto the reaction mechanism, the authors $^{125}$ performed several control experiments. When the reaction was carried out in the presence of TEMPO, neither the desired product nor the alkene-TEMPO adduct were observed; only the alkyl-TEMPO adduct was isolated, confirming the presence of alkyl radicals from the corresponding alkyl trifluoroborate compounds.

When the mixture of (E)-3-(p-tolyl)acrylic acid and BI-OAc (Scheme 52) was monitored by NMR experiments, the formation of a new vinyl carboxylic acid complex was observed. This complex was isolated and characterized by X-ray crystallography. ${ }^{125}$

\section{Scheme 52. Characterization of Benziodoxole Vinyl} Carboxylic Acid Complex

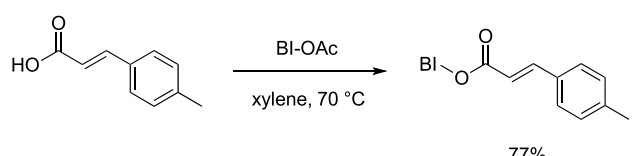

With these results in hand, the authors proposed the reaction mechanism that is illustrated in Scheme 53. The vinyl

Scheme 53. Proposed Mechanism: Photocatalytic Alkenylation Reaction

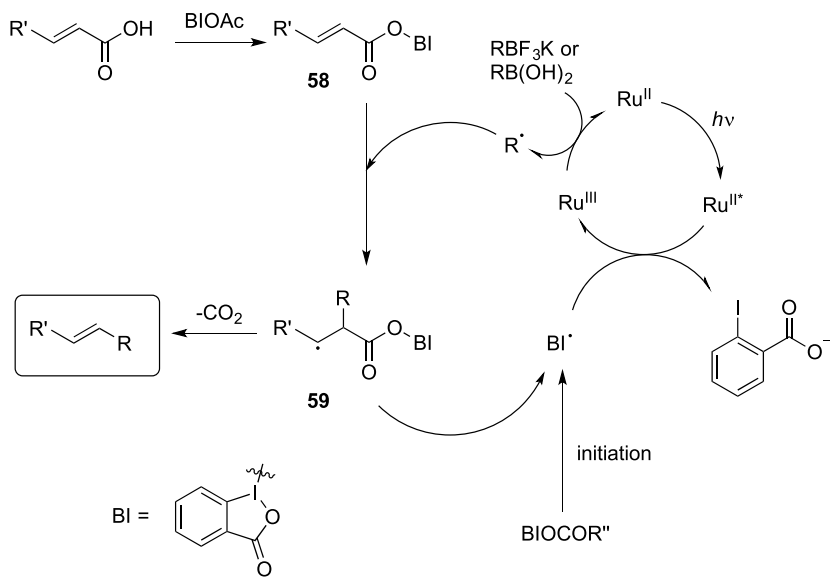

carboxylic acid reacts with BI-OAc to afford benziodoxole vinyl carboxylic acid complex $\mathbf{5 8}$ (Scheme 53), which is oxidized in the initiation step. The trifluoroboronate (or boronic acid) reacts with $\left[\mathrm{Ru}(\mathrm{bpy})_{3}\right]^{2+}$ to generate alkyl radical $\mathrm{R}$ and $\left[\mathrm{Ru}(\mathrm{bpy})_{3}\right]^{3+}$. The $\mathrm{R}$ radical adds to the double bond of complex $\mathbf{5 8}$ (Scheme 53) with formation of radical adduct $\mathbf{5 9}$ (Scheme 53), which undergoes decarboxylation to release the benziodoxole radical and the alkene product. ${ }^{125}$

The authors ${ }^{125}$ also reported the alkenylation of a watersoluble vinyl carboxylic acid (Scheme 54) at $\mathrm{pH} 7.4$ in a phosphate saline buffer to afford the corresponding alkene with

Scheme 54. Photocatalytic Alkenylation of Trifluoroboronate in Aqueous Media

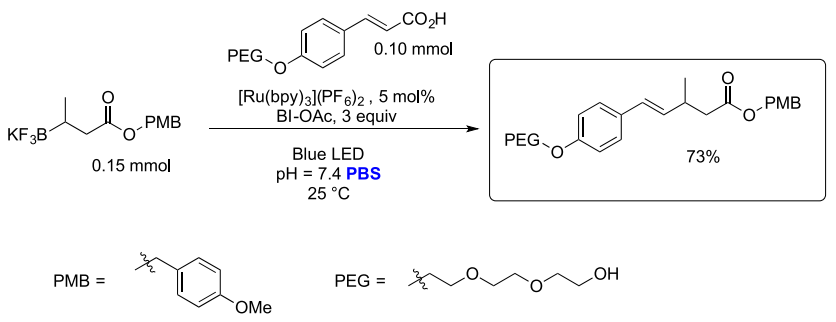


a good yield which probes the potential application in the functionalization of biomolecules in neutral aqueous media.

In another report, Zhang and colleagues ${ }^{126}$ developed a transition metal-free methodology consisting of the consecutive oxidative and decarboxylative acylation/ring expansion of vinylcyclobutanols with $\alpha$-keto acids employing visible light photoredox catalysis.

The reaction mixture consists of $\alpha$-keto acid and vinylcyclobutanol, with Rhodamine B as the photocatalyst, hidroxybenzodioxole $(\mathrm{BI}-\mathrm{OH})$ as the oxidant in a two-phase system of $\mathrm{CH}_{2} \mathrm{Cl}_{2}: \mathrm{H}_{2} \mathrm{O}$ (1:1) under visible light irradiation with a $23 \mathrm{~W}$ CFL lamp. With these conditions in hand, a variety of $\alpha$-keto acids bearing electron-rich or electron-poor groups and several substituted vinylcyclobutanols (Scheme 55) reacted successfully in moderate to good yields. ${ }^{126}$

Scheme 55. $\alpha$-Keto Acid and Vinylcyclobutanols Scope: Metal-Free Oxidative Decarboxylative Acylation/Ring Expansion

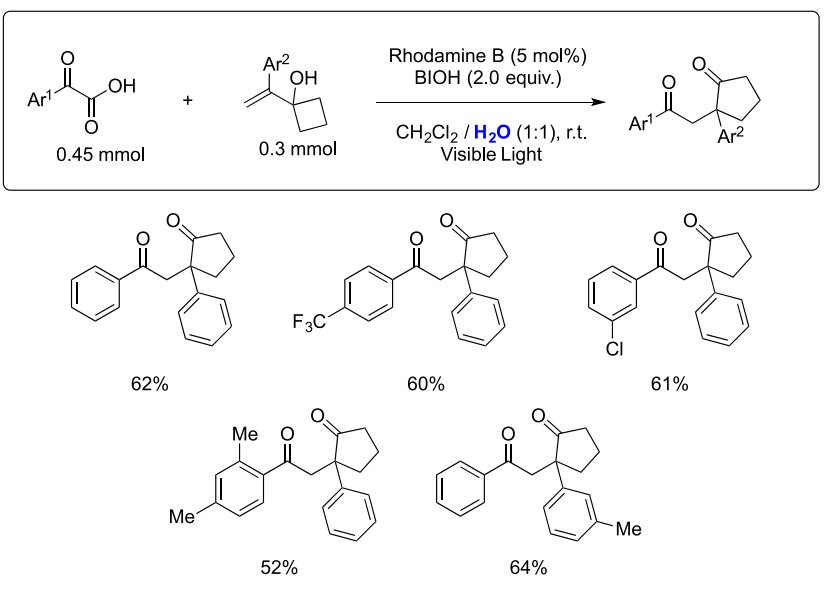

In the proposed mechanism (Scheme 56), hypervalent complex reagent 60 (Scheme 56) generated in situ is oxidized by an excited photocatalyst ( $\mathrm{PC}^{*}$ ) to produce an acyl radical, $\mathrm{CO}_{2}$, and $\mathrm{PC}^{\bullet-}$. The acyl radical adds to vinylcyclobutanol to generate radical adduct 61 (Scheme 56), which reacts with $\mathrm{BI}^{+}$ to afford carbocation $\mathbf{6 2}$ and a BI radical. Intermediate $\mathbf{5 7}$

Scheme 56. Proposed Mechanism: Metal-Free Oxidative Decarboxylative Acylation/Ring Expansion

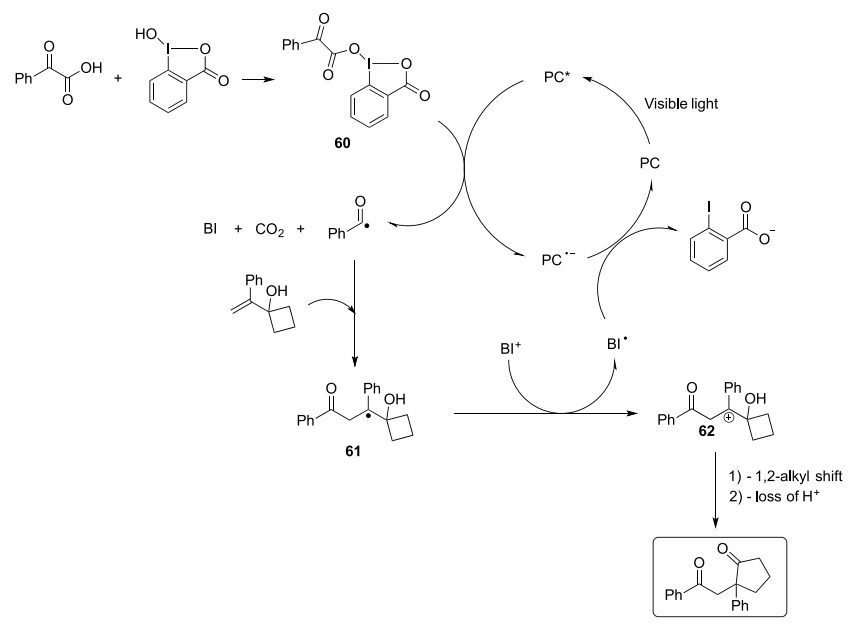

(Scheme 56) undergoes 1,2-alkyl migration to produce the desire product with proton loss. Finally, the BI radical accepts the electron from $\mathrm{PC}^{-}$to regenerate the catalyst. ${ }^{126}$

In control experiments, the acyl radical was evidenced by adding a trapping reagent (TEMPO) to the reaction mixture. Under these conditions, only trace amounts of the desired product was observed, and the acyl-TEMPO adduct was isolated. $^{126}$

\section{PHOTOOXIDATION AND CYCLIZATION REACTIONS IN AQUEOUS MEDIA}

Visible light photocatalysis has shown great potential to initiate oxidation reactions. $^{127}$

Solid polymeric graphitic carbon nitride $\left(\mathrm{g}-\mathrm{C}_{3} \mathrm{~N}_{4}\right)^{128,129}$ is an organic semiconductor with a set of properties such as easy and economic preparation, a band gap less than $2.7 \mathrm{eV}$, absorption of light in the visible region $(<450-460 \mathrm{~nm}){ }^{130}$ and the capacity to activate molecular oxygen. ${ }^{128,131-134}$ All these properties make this a suitable option to mediate photoredox catalytic transformations under visible light irradiation conditions. ${ }^{135}$

$\mathrm{Hu}$ and co-workers ${ }^{135}$ have reported a metal-free heterogeneous photocatalytic system for aerobic oxidation of benzylic $\mathrm{C}-\mathrm{H}$ bonds. The reaction was conducted in the presence of the organic substrate, $\mathrm{g}-\mathrm{C}_{3} \mathrm{~N}_{4}$ as the photocatalyst, oxygen as the oxidizing species, and water as the solvent and under blue light irradiation at room temperature.

As shown in Scheme 57, the optimal reaction conditions were applied to the oxidation of isochromans and phthalans to afford the corresponding 2-isochromanones and phtalides with good yields, respectively. ${ }^{135}$

Scheme 58 summarizes the results of the oxidation of different alkylarenes to ketones. The authors ${ }^{135}$ also reported the oxidation of isoindolines and tetrahydroisoquinolines in DMSO as the reaction solvent.

To demonstrate the practical application of the heterogeneous catalysis, a recycling procedure was established. The photocatalyst was recovered after a reaction with isochromans and subsequently reused. In these cases, the photocatalyst maintains a high photocatalytic activity. ${ }^{135}$

Alcohols can be oxidized to aldehydes and ketones in water under visible light irradiation through the assistance of a 3mercaptopropionic acid (MPA)-capped CdSe quantum dot (MPA-CdSe QD) and $\mathrm{NiCl}_{2}$ without any external oxidant. ${ }^{136}$ The scope of the reaction is briefly depicted in Scheme 59.

Although photocatalyzed cyclization reactions in water or aqueous media were discussed in the Homolytic Aromatic Substitutions with Ulterior Cyclizations section through the homolytic aromatic substitution mechanisms, we, in this section, discuss cyclization reactions involving $\pi$ systems other than aromatics (double and triple bonds).

Tertiary amines can be oxidized to reactive iminium ions or azomethine ylides in the presence of water. On the other hand, secondary amines can be oxidized to imine intermediates leading to reactive electrophiles. ${ }^{137-139}$

Hue and collaborators ${ }^{140}$ attempted to oxidize $N$-alkylsubstituted arylhydroxylamines to nitrones by photocatalysis in water and single electron oxidation, with the aim to realize an ulterior $[3+2]$ cycloaddition reaction of these nitrone intermediates with alkenes, according to a proposed Scheme 60.

After a series of optimization attempts, including solvents, additives, reaction times and stoichiometry, the authors ${ }^{140}$ 


\section{Scheme 57. Photocatalyzed Aerobic Oxidation of Isochromans and Phthalans}

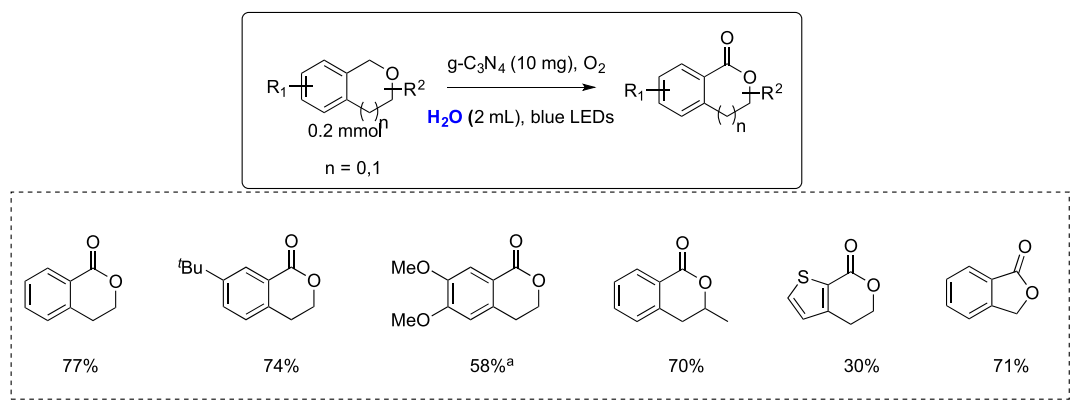

Scheme 58. Photocatalyzed Aerobic Oxidation of Isochromans and Phthalans

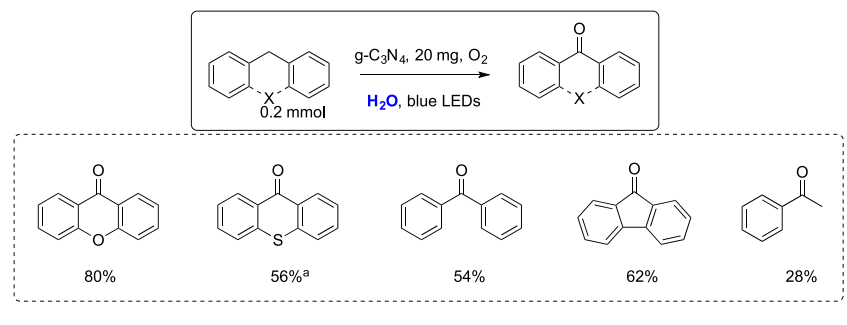

Scheme 59. Oxidation of Alcohols to Aldehydes and Ketones

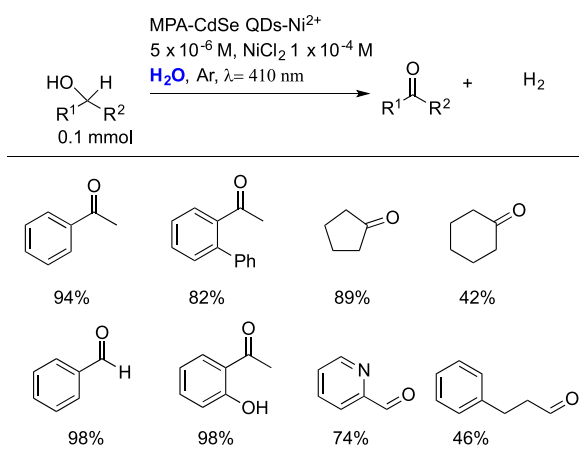

Scheme 60. Synthesis Design

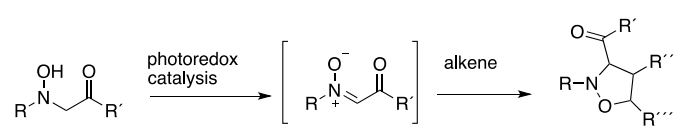

achieved proper conditions for the $[3+2]$ cycloaddition of these nitrone intermediates with alkenes consisting of an arylalkyl hydroxylamine $(0.1 \mathrm{mmol})$ substrate, alkene $(0.3-0.4$ $\mathrm{mmol}),\left[\operatorname{Ir}(\mathrm{ppy})_{2} \mathrm{bpy}_{3} \mathrm{PF}_{6}\right.$ as the photocatalyst (1 mol \%), the presence of water (2.5 equiv) in ethyl acetate as the solvent, and irradiation with an $11 \mathrm{~W}$ fluorescent bulb for $24 \mathrm{~h}$ at room temperature. The scope of the transformation is illustrated in Scheme 61.

Different ester groups such as $-\mathrm{CO}_{2} \mathrm{Me},-\mathrm{CO}_{2} \mathrm{Et},-\mathrm{CO}_{2}{ }^{i} \mathrm{Pr}$, and $-\mathrm{CO}_{2}{ }^{t} \mathrm{Bu}$ were included in the starting $N$-alkylsubstituted arylhydroxylamines under the current reaction conditions. In all cases, high yields and diastereoselectivities were encountered for the respective $N$-phenylisoxazolidines. ${ }^{140}$

The authors ${ }^{140}$ also inspected the scope of the reaction in relation to the aryl ring attached to the $\mathrm{N}$ atom, according to Scheme 62.
Scheme 61. Selected Examples for Evaluation of Different Ester Groups and Dipolarophiles in Photoredox Oxidative Cyclization Reaction of Nitrones and Alkenes

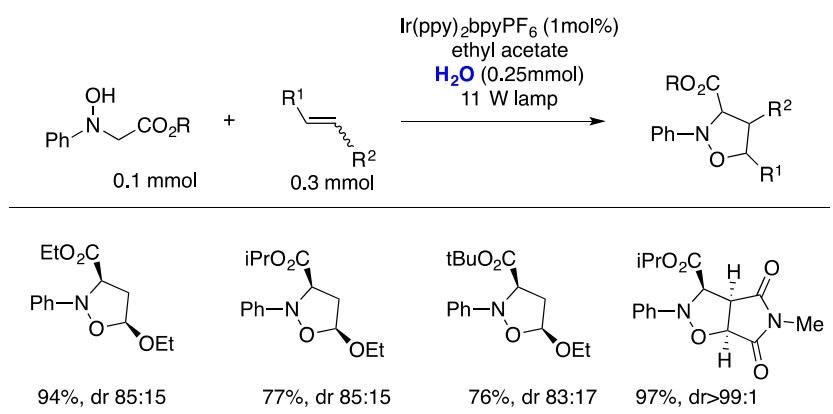

Scheme 62. Selected Examples for Evaluation of Different Aryl Groups and Dipolarophiles in Photoredox Oxidative Cyclization Reaction of Nitrones and Alkenes

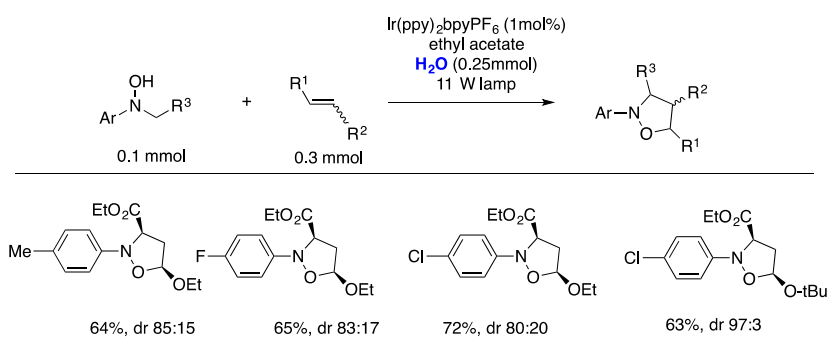

As observed from Scheme 62, hydroxylamines substituted with aryl rings bearing $\mathrm{Me}, \mathrm{F}$, and $\mathrm{Cl}$ groups resulted in good yields and diastereoselectivities.

The authors ${ }^{140}$ proposed a reaction mechanism such as that depicted in Scheme 63. Upon irradiation, the photocatalyst is converted to $\mathrm{Ir}^{3 *+}$ and quenched through a reductive ET by hydroxylamine 63 to produce $\operatorname{Ir}^{2+}$ and ammonium radical cation 64. The $\mathrm{Ir}^{3+}$ photocatalyst is regenerated through oxidative ET from oxygen to yield $\mathrm{O}_{2}{ }^{*-}$. Also, photoredox catalysis can provoke the generation of active species such as $\mathrm{HO}^{*}, \mathrm{HO}^{-}$, and $\mathrm{H}_{2} \mathrm{O}_{2}$ from water. ${ }^{141,142}$ In the case of the reactions above, the authors claimed that the presence of oxygen is the main source of the reactive oxygenated species.

Upon deprotonation, radical cation 64 can form either radical 65 or 66 (a carbon-centered radical or oxygen-centered radical, respectively). Electron transfer from 65 affords intermediate 67 , which can be deprotonated by $\mathrm{HOO}^{-}$to nitrone 68. A reaction of nitrone 68 with alkene 69 affords [3 +2 ] cycloaddition product 70 (Scheme 63).

Kumar and colleagues ${ }^{143}$ have developed a visible lightinduced dehydrogenative trifluoromethylation and oxidation of 


\section{Scheme 63. Proposed Reaction Mechanism}
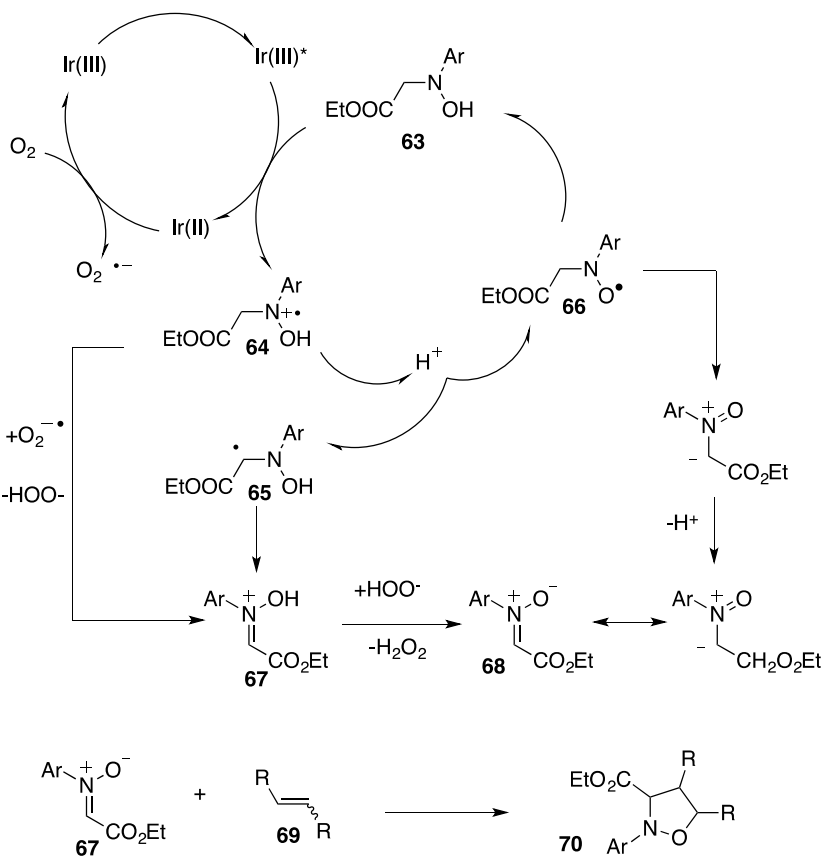

1,6-enynes with water, starting from a 1-(phenylethynyl)-2(vinyloxy)benzene substrate toward the synthesis of trifluoromethylated C3-aryloylated benzofurans, benzothiophenes, and indoles. The authors found optimum reaction conditions consisting of the use of (1-(phenylethynyl)-2-(vinyloxy)benzene $(0.1 \mathrm{mmol})$ as the substrate, 1,10-phenanthroline5,6-dione as the photocatalyst $(0.01 \mathrm{mmol})$, and a Langlois reagent $\mathrm{CF}_{3} \mathrm{SO}_{2} \mathrm{Na}(0.3 \mathrm{mmol})$ in acetonitrile:water (9:1) as the solvent mixture in the presence of visible light.

The scope of the reaction ${ }^{143}$ is illustrated in Scheme 64.

Scheme 64. Selected Examples for Construction of Trifluoromethylated C3-Aryloylated Benzofurans and Benzothiophenes

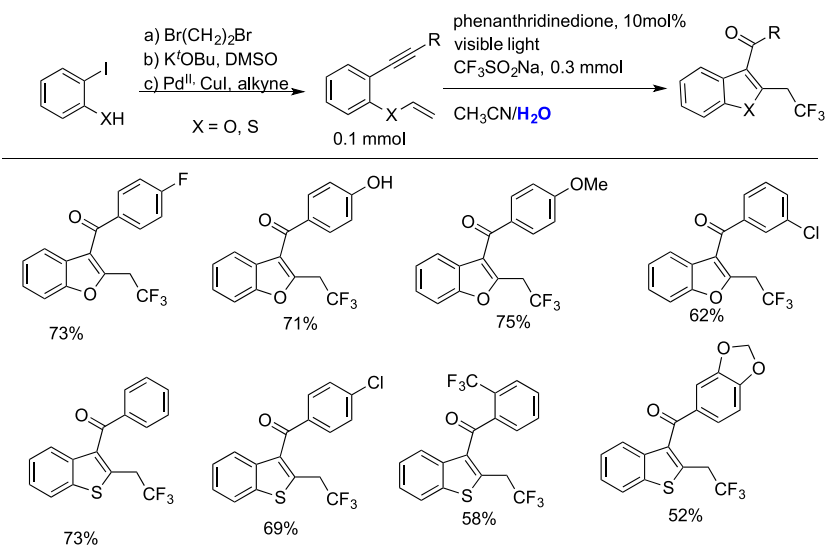

From Scheme 64, it is observed that both 2-iodophenols and 2-iodothiophenols can be used as substrates to yield 2-benzoylsubstituted benzofurans and benzo[ $[b]$ thiophenes in good yields. Also, starting from 2-iodoanilines, trifluoromethylated C3-aryloylated indoles are synthesized in reasonably good yields (Scheme 65). The reaction employs the Langois reagent and 4-methyl- $\mathrm{N}$-(2-(arylethynyl)phenyl)- $\mathrm{N}$-vinylbenzenesulfo-
Scheme 65. Selected Examples for Construction of Trifluoromethylated C3-Aryloylated Indoles

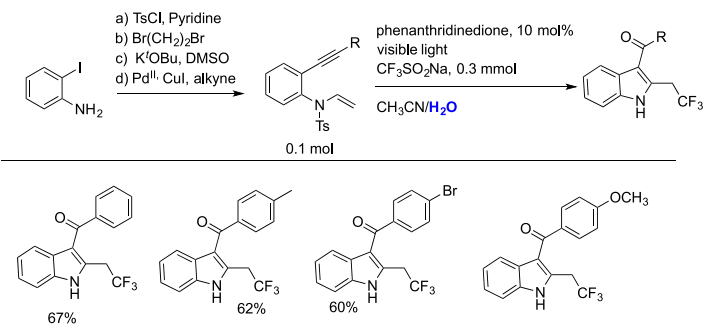

namide under a phenanthridinedione photocatalyst in $\mathrm{MeCN}: \mathrm{H}_{2} \mathrm{O}$ as the solvent mixture. ${ }^{143}$

As observed from Scheme 65, aryl groups with 4-methyl, $\mathrm{H}$, 4- $\mathrm{Br}$, and $4-\mathrm{OCH}_{3}$ were tested, providing good yields of the indole products.

The authors ${ }^{143}$ performed a series of experiments in order to provide light to the reaction mechanism. When the reaction was undertaken in $\mathrm{H}_{2}{ }^{18} \mathrm{O}$, the formation of $\mathrm{O}^{18}$-labeled trifluoromethylated C3-aryloyl benzofuran was found, indicating that oxygen in product arises from water. Also, the reaction in $\mathrm{D}_{2} \mathrm{O}$ showed a NMR signal at $\delta=4.55 \mathrm{ppm}$ and $\mathrm{J}=42.8 \mathrm{~Hz}$, consistent with $\mathrm{H}_{2}$ evolution according to Scheme 66 .

Scheme 66. Labeling Experiments

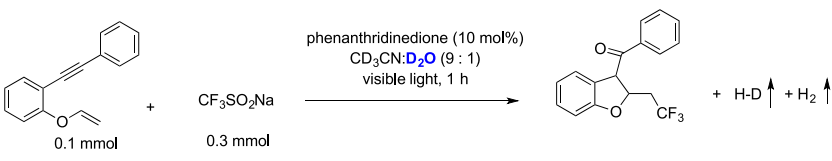

The authors ${ }^{143}$ also investigated the reaction pathway employing ESR experiments. In the presence of 2-methyl-2nitrosopropane (MNP), a dimer resulted in the trapping of $\mathrm{CF}_{3}$ radicals, observing the tert-butyl-trifluoromethyl nitroxide radical. Also, in the presence of TEMPO, a radical scavenger, the product was not found, and the adduct TEMPO- $\mathrm{CF}_{3}$ was encountered. From redox parameters, the excited state redox potential of the photocatalyst 1,10-phenanthroline-5,6-dione (PQ) $\mathrm{E}_{\mathrm{red}}{ }^{1 / 2} *\left({ }^{3} \mathrm{PQ}^{*} / \mathrm{PQ}^{-}\right)$is $+1.6 \mathrm{~V}^{1}$ and that of the Langlois reagent is $+1.05 \mathrm{~V}$ (vs SCE), ${ }^{144}$ which warrants the electron transfer process (meaning that PQ is strong enough to oxidize the Langlois reagent by electron transfer).

With the mechanistic probe experiments in hand, the authors ${ }^{143}$ postulated the following reaction mechanism, according to Scheme 67.

Upon excitation of the photocatalyst $P Q$ its excited triplet excited state is able to oxidize the Langlois reagent to $\mathrm{CF}_{3}$ radicals, which add in turn to substrate 71 to produce radical intermediate 73 , which is oxidized by radical $\mathrm{PQH}$ to yield cation intermediate 73. Upon internal cationic cyclization of 73, vinylic intermediate 74 is generated, which upon water nucleophilic addition renders intermediate 76 . Ulterior deprotonation and hydrogen evolution gives final product 78. An alternative radical pathway intermediate can be conceived through 75 . $^{143}$

\section{MISCELLANEOUS PHOTOREDOX REACTIONS IN AQUEOUS MEDIA}

Condensation Reactions. Hossein and colleagues ${ }^{145}$ established a protocol for the synthesis of substituted pyrazines 


\section{Scheme 67. Proposed Reaction Mechanism}

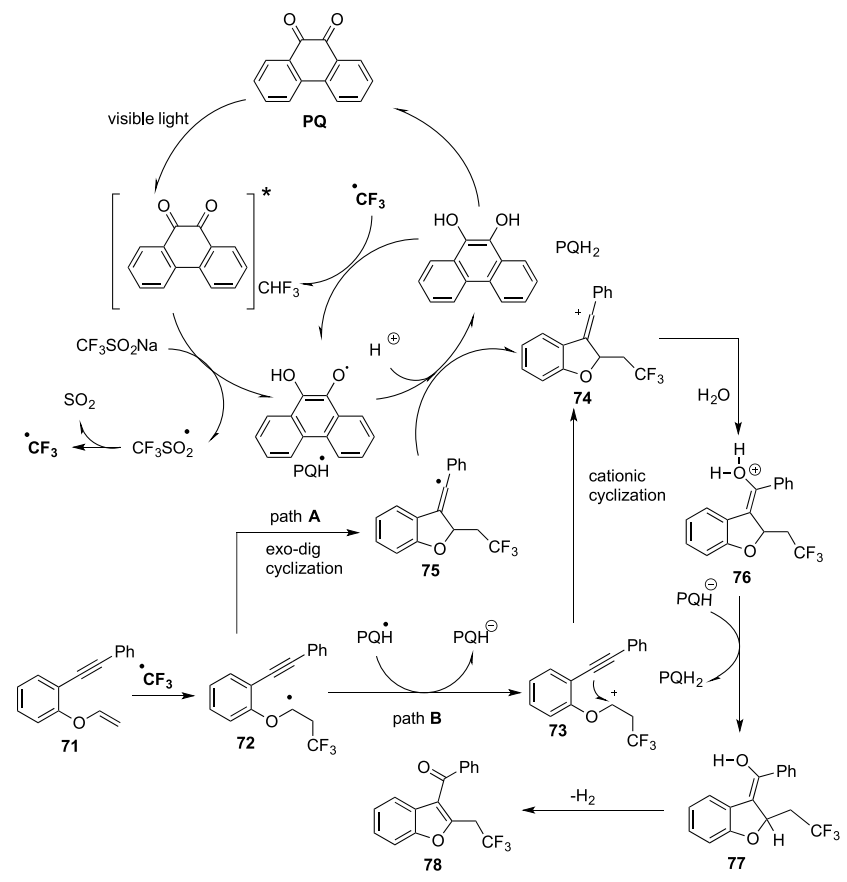

from vinyl azides made possible by a dual-energy and electrontransfer strategy by visible light photocatalysis in aqueous medium.

The best reaction conditions include the use of vinyl azide (0.5 mmol), photocatalyst $\mathrm{Ru}(\mathrm{bpy})_{3} \mathrm{Cl}_{2}(1 \mathrm{~mol} \%)$, and irradiation with a $455 \mathrm{~nm}$ LED in a $\mathrm{CH}_{3} \mathrm{CN}$ :water 4:1 mixture in open air. The scope of the transformation is shown in Scheme 68. Further optimization studies revealed that the addition of another oxidant such as $\mathrm{K}_{2} \mathrm{~S}_{2} \mathrm{O}_{8}$ did not provide any product indicating the importance of oxygen. ${ }^{145}$

Scheme 68. Scope of Synthesis of Substituted Pyrazines
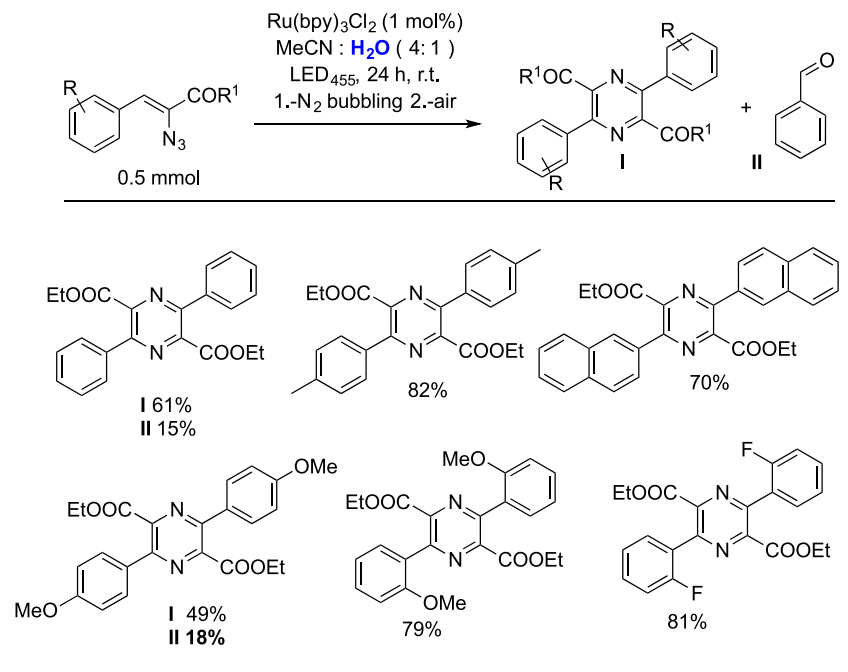

As observed from Scheme 68, aryl vinyl azides substituted with both electron-donating groups $\left(\mathrm{MeO}, \mathrm{CH}_{3}\right)$ or electronneutral groups (H, F) afforded good yields of substituted pyrazines

The authors ${ }^{145}$ investigated the reaction mechanism, as suggested in Scheme 69. The mechanism starts with an energy

\section{Scheme 69. Proposed Reaction Mechanism}

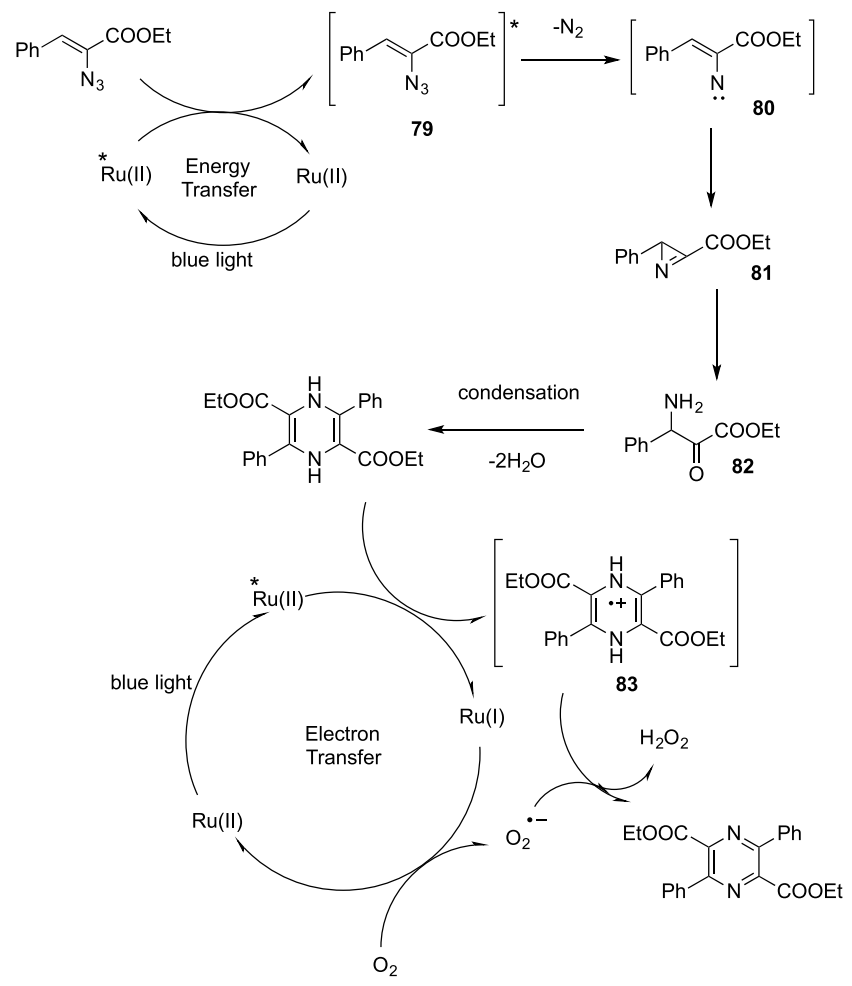

transfer process from the excited $\mathrm{Ru}(\mathrm{II})^{*}$ photocatalyst to vinyl azide. Vinyl azide is converted into its triplet manifold 79, which upon loss of nitrogen forms a reactive nitrene intermediate $\mathbf{8 0}$ that rearranges to the azirine $\mathbf{8 1}$. This azirine 81 may undergo ring opening by water, leading to the formation of $\alpha$-amino ketone $\mathbf{8 2}$. The condensation of $\mathbf{8 2}$ to form dihydropyrazine species. This dihydro species is aromatized by an electron transfer process from $\mathrm{Ru}(\mathrm{II}) *$, yielding radical cation 83 and $\mathrm{Ru}(\mathrm{I})$. Species $\mathbf{8 3}$, upon reaction with an anion superoxide, affords a pyrazine compound and concludes the reaction mechanism. ${ }^{14}$

Synthesis of Boronates and Borylation Reactions. Li and collaborators ${ }^{146}$ presented a photocatalytic strategy for the preparation of alkyl boronates from aliphatic esters in an aqueous reaction media. This transformation is very innovative because borylation reactions usually require anhydrous conditions. In a typical experiment, a primary carboxylic acid-derived $\mathrm{N}$-acyloxyphthalimide, bis(pinacolato)diboron $\left(\mathrm{B}_{2} \mathrm{pin}_{2}\right)$, and catalyst $\left[\operatorname{Ir}(\mathrm{ppy})_{2} \mathrm{dtbpy} \mathrm{PF}_{6}\right.$ are irradiated under visible light in a mixture of DMF: $\mathrm{CH}_{3} \mathrm{CN}: \mathrm{H}_{2} \mathrm{O}$ $(1: 1: 1)$ as the reaction medium (Scheme 70). The authors found this transformation to be useful for efficiently converting several primary $\mathrm{N}$-acyloxyphthalimides with various electronwithdrawing, electron-donating, and electron-neutral groups; alkenes with $(Z)$-configuration as well as terminal alkynes, aryl bromides, and alkyl chlorides were all well tolerated (Scheme 70). ${ }^{146}$

The authors ${ }^{146}$ proved the radical nature of the reaction by performing radical cascade experiments and proposed a conceivable reaction pathway (Scheme 71). Upon light excitation, the $\mathrm{Ir}^{(\mathrm{III})}$ photocatalyst performs a single electron transfer to $N$-acyloxyphthalimide affording the Ir $^{\text {(IV) }}$ photocatalyst and the $N$-acyloxyphthalimide radical anion which suffers homolytic cleavage yielding a carboxyl radical and a phthalimide anion (Scheme 71). The carboxyl radical 
Scheme 70. Selected Examples for Decarboxylative Borylation of Primary Carboxylic Acid-Derived NAcyloxyphthalimide under Visible Light Photoredox Conditions Employing a Mixture of DMF: $\mathrm{CH}_{3} \mathrm{CN}: \mathrm{H}_{2} \mathrm{O}$ (1:1:1) as Reaction Media

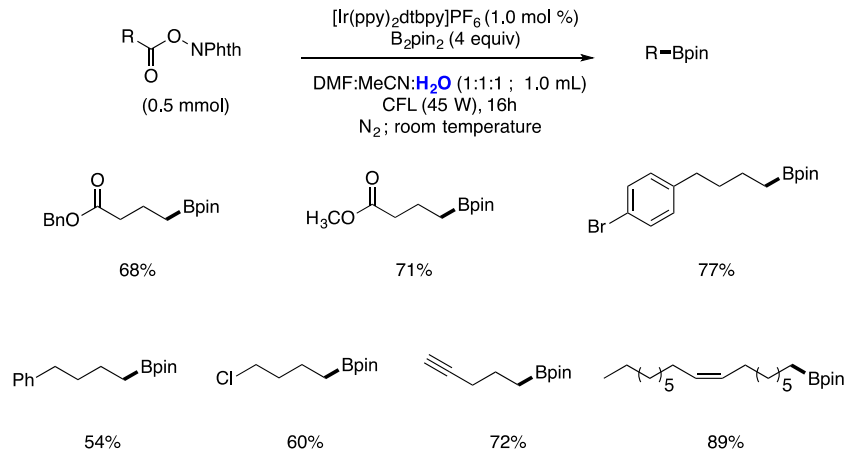

Scheme 71. Proposed Reaction Mechanism for Decarboxylative Borylation of Primary Carboxylic AcidDerived $\mathrm{N}$-Acyloxyphthalimide under Visible Light Photoredox Conditions Employing a Mixture of DMF: $\mathrm{CH}_{3} \mathrm{CN}: \mathrm{H}_{2} \mathrm{O}(1: 1: 1)$ as Reaction Media

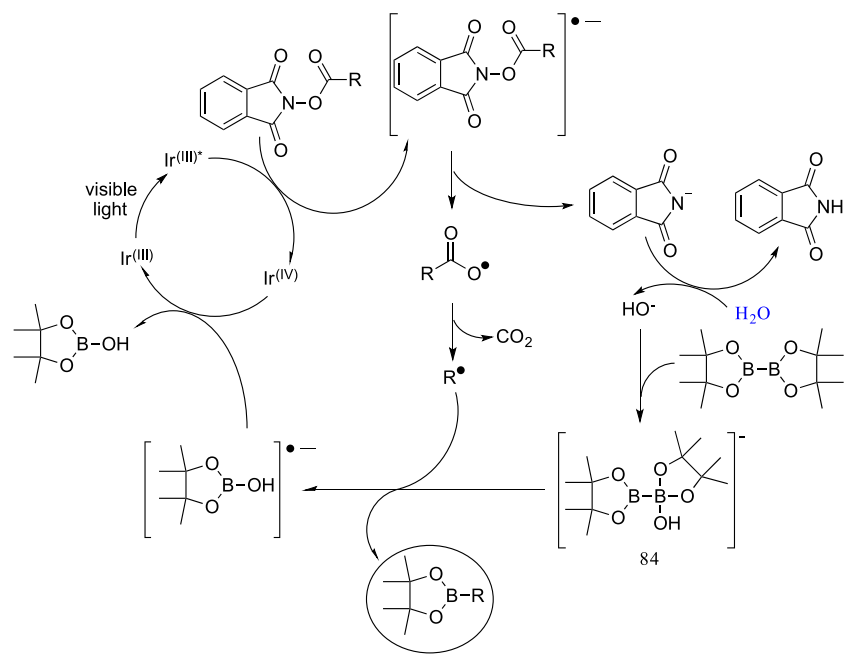

undergoes decarboxylation generating $\mathrm{CO}_{2}$ and an alkyl radical (Scheme 71). The phthalimide anion in the presence of water undergoes protonation generating a hydroxide ion that reacts with $\mathrm{B}_{2}$ pin $_{2}$ affording $\mathrm{sp}^{3}-\mathrm{sp}^{2}$ diboron anion 84 (Scheme 71 ). The alkyl radical reacts with anion 84 yielding the desired reaction product and a boryl radical anion that performs a single electron transfer with the $\mathrm{Ir}^{(\mathrm{IV})}$ photocatalyst regenerating the $\mathrm{Ir}^{(\mathrm{III})}$ photocatalyst and closing the catalytic cycle (Scheme 71). ${ }^{146}$

The borylation of diazonium salts with diboronate esters employing nontoxic carbon dots (CDs) as photocatalysts was recently carried out in water by $\mathrm{Wu}$ and colleagues. ${ }^{147}$ The blue CDs were synthesized through reaction of 2,6diaminopyridine with acrylic acid in the presence of $\mathrm{ZnCl}_{2}$ in tetraethylene glycol as the solvent. ${ }^{147} \mathrm{~A}$ general reaction is illustrated in Scheme 72. This result (Scheme 72) has been discussed in a review article. ${ }^{6}$

$\mathrm{C}-\mathrm{P}$ Bond Formation. The formation of $\mathrm{C}-\mathrm{P}$ bonds is relevant as organophosphorus compounds have many applications in pharmaceutical chemistry or are employed as ligands or intermediates in synthetic organic chemistry. ${ }^{148}$
Scheme 72. Borylation of Diazonium Salts

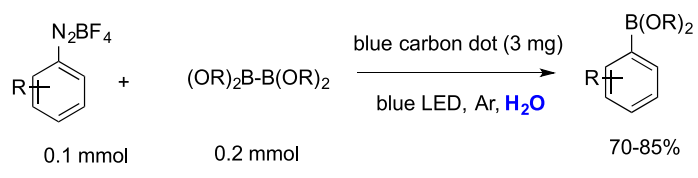

In 2011, Rueping and colleagues ${ }^{149}$ developed the first photoredox catalytic protocol for the oxidative carbonphosphorus bond formation. The reaction has even more relevance from the perspective of the aqueous reaction medium employed, which brings useful implications in biological aqueous systems. The protocol employs $\mathrm{N}$-aryltetrahydroisoquinolines and dialkyl (or aryl) phosphites as substrates and $\left[\operatorname{Ir}(\mathrm{ppy})_{2}(\mathrm{bpy})\right]\left[\mathrm{PF}_{6}\right]$ as the catalyst in a mixture of toluene and water as the reaction medium under visible light irradiation with a $5 \mathrm{~W}$ fluorescent lamp.

The scope of the reaction is shown in Scheme 73. The authors $^{149}$ also investigated several phosphite esters, with

Scheme 73. Visible Light-Mediated Oxidative Phosphonylation

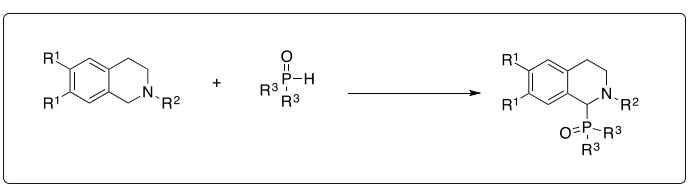

$R^{1}=H, R^{2}=P h, R^{3}=O E t$

$\mathrm{R}^{1}=\mathrm{H}, \mathrm{R}^{2}=P+-\mathrm{Ph}, \mathrm{R}^{3}=\mathrm{OEt}$
$\mathrm{R}^{1}=\mathrm{H}_{\mathrm{B}} \mathrm{R}^{2}=p \mathrm{Br}-\mathrm{Ph}, \mathrm{R}^{3}=\mathrm{OE}$

$\mathrm{R}^{1}=\mathrm{H}, \mathrm{R}^{2}=p \mathrm{Br}-\mathrm{Ph}, \mathrm{R}^{3}=\mathrm{O}$
$\mathrm{R}^{1}=\mathrm{H}, \mathrm{R}^{2}=\mathrm{Ph}, \mathrm{R}^{3}=\mathrm{OPh}$

$\mathrm{R}^{1}=\mathrm{H}, \mathrm{R}^{2}=p \mathrm{~F}-\mathrm{Ph}, \mathrm{R}^{3}=\mathrm{OPh}$

$\mathrm{R}^{1}=\mathrm{H}_{1} \mathrm{R}^{2}=p \mathrm{Br}-\mathrm{Ph}, \mathrm{R}^{3}=\mathrm{OPh}$
$\mathrm{R}^{1}=\mathrm{H}, \mathrm{R}^{2}=\mathrm{Ph}, \mathrm{R}^{3}=\mathrm{OPr}$

$89 \%$
$61 \%$
$75 \%$
$91 \%$
$78 \%$
$90 \%$
$39 \%$

different alkyl groups. The phosphite with the bulky groups (as $-\mathrm{O}^{i} \mathrm{Pr}$ ) reacted with the isoquinolinium ion intermediate generated photochemically under more prolonged reaction times and with poor yields. The dimethyl phosphite did not provide the product, probably due to the fast hydrolysis reaction of the dimethyl phosphite in the presence of water.

The resulting $\alpha$-amino phosphonates are interesting compounds in medicinal chemistry with a myriad of applications, such as $\alpha$-amino acids analogues, enzyme inhibitors, and present antibacterial and antifungal activity. ${ }^{150}$

Other interesting examples for the construction of $\mathrm{C}-\mathrm{P}$ bonds by photocatalysis in water followed. The direct synthesis of $Z$-alkenylphosphine oxides occurred through the addition of phosphoryl radicals to alkynes ${ }^{6,151}$ (Scheme 74A); also by this method, the syntheses of 3-phosphorylated thioflavones, ${ }^{6,152}$ (Scheme 74B) and 3-phosphorylated benzothiophenes, ${ }^{6,153}$ (Scheme 74C) have been attempted. All these methods have been recently reviewed and are succinctly summarized in Scheme $74 .^{6}$

Debromination and Bromination Reactions. Willner and co-workers ${ }^{154}$ have reported the photochemical debromination of 1,2-dibromoarylethanes to afford the corresponding 1,2-diarylethylenes using a heterogeneous system. For that purpose, they employed an alkylviologen, $N, N^{\prime}$-dioctyl-4, $4^{\prime}$ bipyridiniumdibromide $\left(\mathrm{C}_{8} \mathrm{~V}^{+}\right)$as an electron carrier, due its solubilization properties (which depend on its oxidation state).

The two-phase system is composed of an organic solution of a dihalogenated substrate in ethyl acetate and an aqueous solution of ruthenium tris(bipyridine) dichloride $\left(\mathrm{Ru}(\mathrm{bpy})_{3}{ }^{2+}\right)$, $\left(\mathrm{NH}_{4}\right)_{3} \mathrm{EDTA}$, and $\mathrm{C}_{8} \mathrm{~V}^{+}$. The reaction mixture was irradiated 
Scheme 74. Photocatalyzed Syntheses of Phosphorylated ZAlkenylphosphine oxides (A), 3-Phosphorylated Thioflavones (B), and 3-Phosphorylated Benzothiophenes (C)

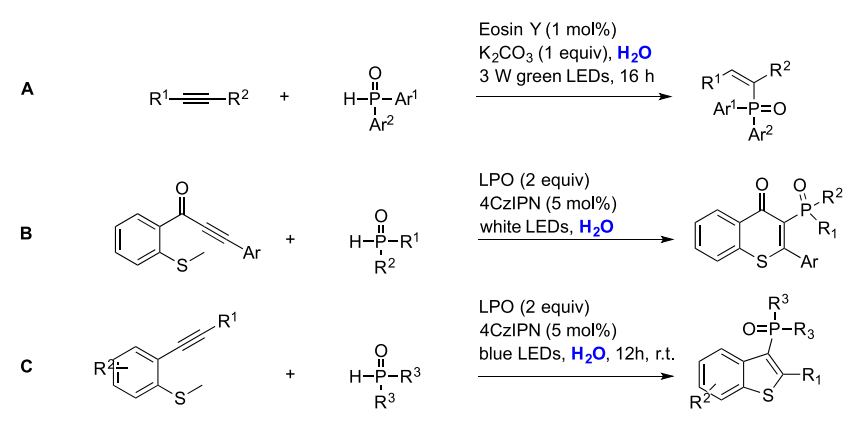

with a $1000 \mathrm{~W}$ halogen-quartz lamp employing a filter $(\lambda>$ $400 \mathrm{~nm})$.

Upon irradiation of the biphasic system (Scheme 75), excited $\mathrm{Ru}(\text { bpy })_{3}{ }^{2+}$ is quenched by $\mathrm{C}_{8} \mathrm{~V}^{+}$(soluble only in

Scheme 75. Photocatalytic Reduction of 1,2Dibromostilbene in a Two-Phase System Mediated by $\mathrm{C}_{8} \mathrm{~V}^{2+}$

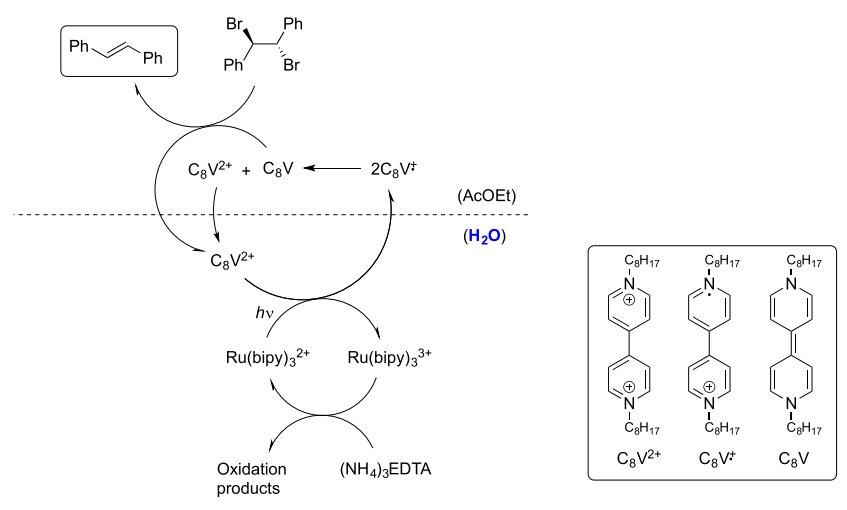

aqueous media) affording $\mathrm{Ru}(\mathrm{bpy})_{3}{ }^{3+}$ and $\mathrm{C}_{8} \mathrm{~V}^{+} \bullet$ This reduced form $\left(\mathrm{C}_{8} \mathrm{~V}^{+}\right)$, with lipophilic properties, is extracted into the organic phase. The active two-electron reducing agent $\mathrm{C}_{8} \mathrm{~V}$ is formed in a disproportionation reaction (eq 1 ).

$$
2 \mathrm{C}_{8} \mathrm{~V}^{+\bullet} \rightleftharpoons \mathrm{C}_{8} \mathrm{~V}^{2+}+\mathrm{C}_{8} \mathrm{~V}
$$

The disproportionation reaction of $\mathrm{C}_{8} \mathrm{~V}^{+} \bullet$ affords $\mathrm{C}_{8} \mathrm{~V}^{2+}$ (which is re-extracted into the aqueous phase) and $\mathrm{C}_{8} \mathrm{~V}$, which is capable of reducing the dibromide. Finally, the debromination reaction recycles the mediating electron acceptor, and $\mathrm{Ru}(\text { bpy })_{3}{ }^{2+}$ is regenerated by oxidation of $\left(\mathrm{NH}_{4}\right)_{3}$ EDTA.

Using this system, the authors ${ }^{154}$ achieved the conversion of several 1,2-dibromoarylethanes into the corresponding transstilbenes, as shown in Scheme 76. No reduction of dibromides occurs when $\mathrm{C}_{8} \mathrm{~V}^{2+}$ is excluded from the reaction mixture.

Because the reducing species $\mathrm{C}_{8} \mathrm{~V}^{2+}$ is extracted into the aqueous phase after formation, the disproportionation reaction balance is shifted toward products. In fact, such a debromination reaction should be unfavorable in a homogeneous solution due to a low disproportionation constant $\left(K_{\mathrm{d}}=\right.$ $\left.5 \times 10^{-8}\right)$. Indeed, the irradiation of an acetonitrile mixture of $\mathrm{Ru}(\mathrm{bpy})_{3}{ }^{2+}$ as the sensitizer, $\mathrm{C}_{8} \mathrm{~V}^{+}$as the electron carrier, triethanolamine as the electron donor, and 1,2-dibromostilbene does not result in the formation of a debromination
Scheme 76. Product Yields: Debromination of 1,2Dibromoarylethanes Mediated by $\mathrm{C}_{8} \mathrm{~V}^{2+}$ in a Two-Phase System

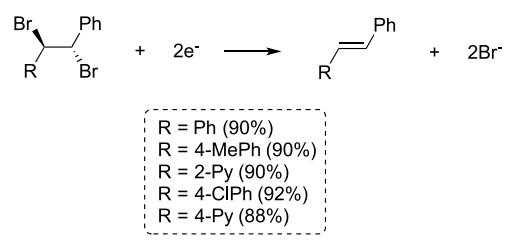

product, which emphasizes the relevance of water in this photocatalytic reaction.

The authors also reported ${ }^{155}$ a remarkably similar cyclic chemical debromination of meso-1,2-dibromostilbene in a twophase system. In that case, the chemical initiation is accomplished with the addition of sodium dithionite in the aqueous phase.

Formyloxylation Reactions. Zou and colleagues ${ }^{156}$ achieved the formyloxylation reaction of 3-bromooxindoles in a mixture of water and $N, N$-dimethylformamide (DMF) in the presence of $f a c-\operatorname{Ir}(\mathrm{ppy})_{3}$ as the photoredox catalyst under visible light irradiation at ambient temperature, yielding 3formyloxyoxindoles in high yields. The use of DMF provides the " $\mathrm{H}, \mathrm{C}, \mathrm{O}$ " source, and $\mathrm{H}_{2} \mathrm{O}$ is the extra "O" source. The authors optimized the reaction conditions arriving at the following stoichiometry: 3,5-dibromo-1,3-dimethylindolin-2one as the initial substrate $(0.2 \mathrm{mmol}), \mathrm{DMF}(2 \mathrm{~mL}), \mathrm{H}_{2} \mathrm{O}$ (10 equiv), and $f a c-\operatorname{Ir}(\text { ppy })_{3}(2 \mathrm{~mol} \%)$ for $2.5 \mathrm{~h}$. The scope of the transformation is illustrated in Scheme 77.

Scheme 77. Selected Examples for Formyloxylation Reaction of 3-Bromooxindoles with Water and $\mathrm{N}, \mathrm{N}$ Dimethylformamide

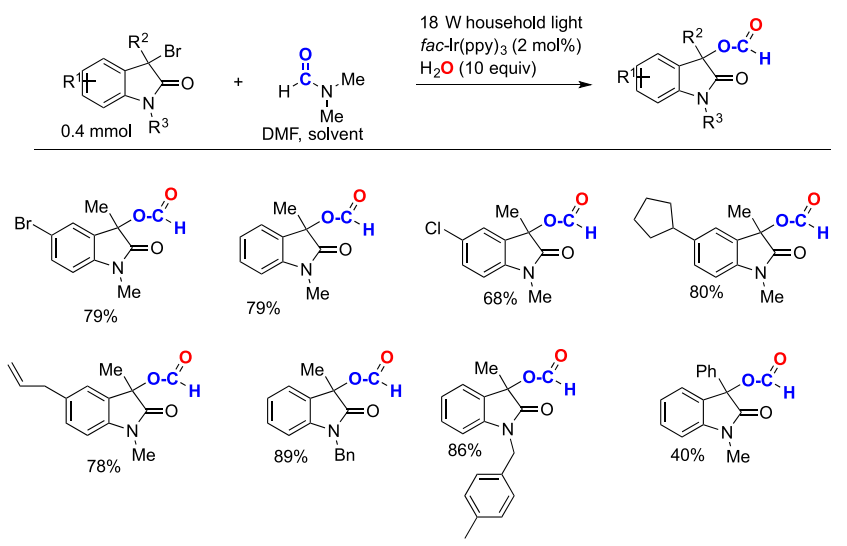

As observed from Scheme 77, 3-bromooxindoles bearing halogen substituents $(\mathrm{Br}, \mathrm{Cl})$ and cycloalkyl substituents (cyclopentyl) afforded good product yields of the respective formylation products.

The authors ${ }^{156}$ also investigated the reaction mechanism. A proposal is depicted in Scheme 78.

In the proposed mechanism (Scheme 78 ), the photocatalyst is excited into a triplet state (metal-to-ligand charge transfer complex, MLCT) capable to reduce by electron transfer substrate 85 to radical 86 . Radical 86 then reacts with DMF to yield radical 87 , which is oxidized by ET by substrate 85 to iminium species 88, which upon reaction with water affords product 90 and dimethylamine amine 89 (Scheme 78). ${ }^{156}$ 


\section{Scheme 78. Proposed Reaction Mechanism}

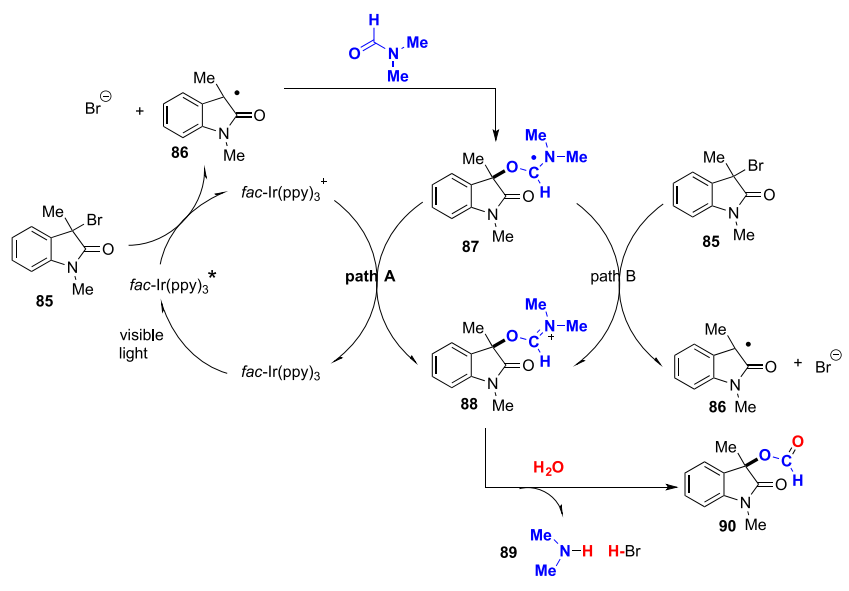

Allylation of Aldehydes. Allylation reactions have been described under Barbier conditions, with the involvement of various metals such as nickel, ${ }^{157,158}$ chromium, ${ }^{159-161}$ titanium, ${ }^{162,163}$ and cobalt. ${ }^{164}$ Bismuth has also been used in allylation reactions under various conditions. ${ }^{165-171}$

Potenti and colleagues ${ }^{172}$ have recently found the photocatalyzed allylation reaction of aldehydes mediated by $\mathrm{Bi}(\mathrm{OTf})_{3}$ (10 mol \%), employing 2,4,6-tri(9H-carbazol-9-yl)5-chloroisophthalonitrile (3CzClIPN, $5 \mathrm{~mol} \%$ ) as the photocatalyst in EtOH: $\mathrm{H}_{2} \mathrm{O}(1: 1)$ as the mixture of solvents, and Hantzsch ester (HE, 2 equiv) as the electron source under blue LED irradiation. The authors ${ }^{172}$ avoided the use of Ir or $\mathrm{Ru}$ photocatalysts. The scope of the reaction was studied, according to Scheme 79.

Scheme 79. Selected Examples for Allylation of Aromatic and Aliphatic Acids
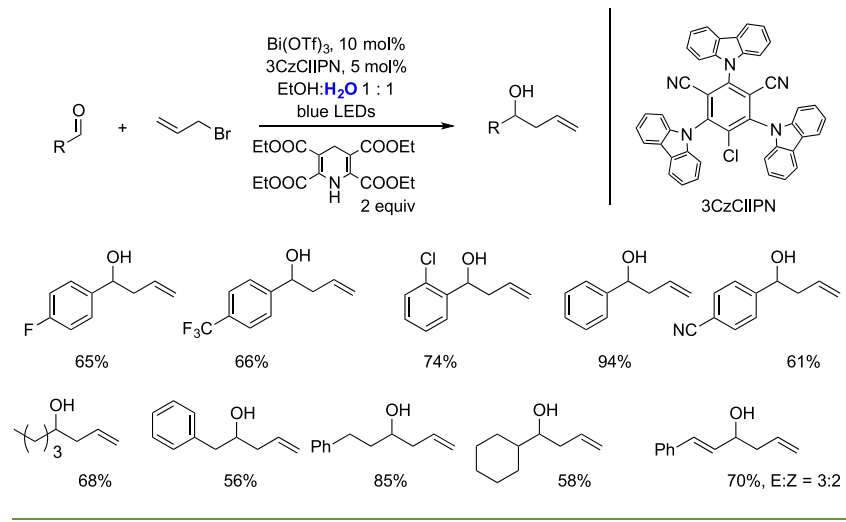

In this way, aromatic aldehydes bearing 4-fluoro, 4- $\mathrm{CF}_{3}$, 2chloro, and 4-cyano substituents were rehearsed, affording good results of the respective benzylic alcohols. Aliphatic aldehydes such as valeraldehyde, 2-phenylacetaldehyde, 3phenylpropanal, cyclohexanecarbaldehyde, and cinnamaldehyde also provide good product yields. ${ }^{172}$

The authors investigated the mechanism of the reaction performing luminescence quenching experiments of the photocatalyst (i.e., 3CzClIPN). In the presence of increasing amounts of $\mathrm{Bi}(\mathrm{OTf})_{3}$ or allyl bromide, no decrease in the emission intensity of $3 \mathrm{CzClIPN}^{*}$ was observed. On the contrary, the addition of 4-chlorobenzaldehyde hardly decreased the intensity of fluorescence, explaining the pinacol coupling in absence of $\mathrm{Bi}(\mathrm{OTf})_{3}$. A similar behavior is observed when the $\mathrm{HE}$ is introduced in the presence of $3 \mathrm{CzClIPN}$. Considering all the probe experiments performed, the authors ${ }^{172}$ proposed the following reaction mechanism (Scheme 80).

Scheme 80. Proposed Reaction Mechanism

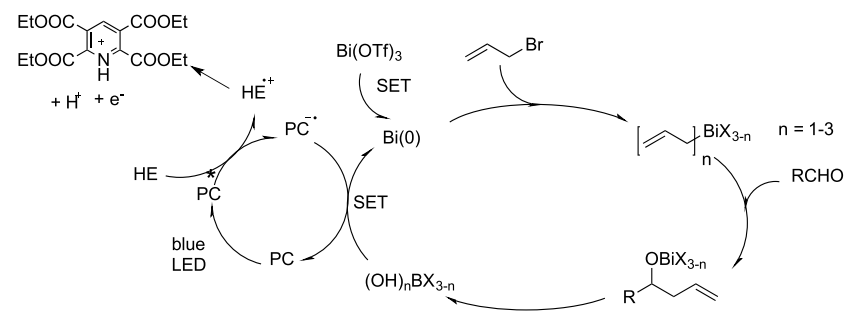

The reductive quenching of $3 \mathrm{CzClIPN}$ by Hantzsch ester (HE) produces the radical cation of $\mathrm{HE}$ (according to redox potentials, $\mathrm{E}_{1 / 2} 3 \mathrm{CzClIPN}^{*} / 3 \mathrm{CzClIPN}^{\bullet-}=+1.56 \mathrm{~V}$ vs $\mathrm{SCE}$ and the oxidation potential of the Hantzsch's ester $\mathrm{E}_{1 / 2} \mathrm{HE}^{*+} /$ $\mathrm{HE}=+1.0$ vs $\mathrm{SCE}^{173}$ purporting that oxidation of $\mathrm{HE}$ is feasible by $\left.3 \mathrm{CzClIPN}^{*}\right)$. The reducing $3 \mathrm{CzClIPN}^{\bullet-}$ species is thus formed, which is able to reduce the $\mathrm{Bi}$ salt to $\mathrm{Bi}(0)$, which in turn complexes with allyl bromide. The insensitive reactions to oxygen and TEMPO rule out a free radical mechanism and formation of allylic radicals. The formation of $\operatorname{Bi}(0)$ should be considered to take place in the reaction mechanism. The insertion of bismuth to the $\mathrm{C}-\mathrm{Br}$ bond could form allylbismuth(III), diallylbismuth(III), and triallylbismuth(III) species as the organometallic intermediates that react with aldehydes to give the corresponding homoallylic alkoxides. The protonated aromatized $\mathrm{HE}$ in the aqueous media allows the facile protonation of a $\mathrm{Bi}$ alkoxide intermediate, permitting the recycling of Bi salts. ${ }^{172}$

\section{CONCLUSIONS}

The array of organic photocatalyzed reactions presented herein in water or aqueous media is encouraging the organic synthetic chemistry community to further explore the potential and advantages inherent in diminishing the environmental impact of the synthetic and photocatalytic processes performed in conventional organic solvents. Many ordinary reaction types have been discussed, such as radical additions to alkenes, ATRA reactions, arylation processes, homolytic aromatic substitutions, and several other standard organic transformations commonly employed for the synthesis of organic scaffolds that are made possible in water or water mixtures by photocatalysis.

One of the problems that chemists face when choosing reaction solvents is the solubility of reactants, as the vast majority of ordinary organic substrates are organic solvent soluble. Even a more critical situation emerges when employing light as a reactant (such as in photocatalysis), as heterogeneous media can interfere with proper photon absorption due to sample or photocatalyst heterogeneous distribution. However, all examples depicted in heterogeneous media or with nonwater-soluble reactants seem to proceed without difficulties, as illustrated in this perspective.

There is no barrier as to the use of any typical photocatalyst employed in organic synthesis when performing a photoreaction in water or aqueous mixtures. Ir- and Ru-polypyridyl complexes have been found to give promising results when 
reactions are carried out in water, as well as with organic dyes such as Rose Bengal, Eosin Y, 1,10-phenanthroline-5,6-dione, or graphitic carbon nitride $\left(\mathrm{g}-\mathrm{C}_{3} \mathrm{~N}_{4}\right)$; quantum dots and carbon dots; or semiconductors such as $\mathrm{TiO}_{2}$ and CdSe. Parallel studies on the photocatalyst efficiency in organic media and water should be addressed in those photocatalyzed reactions that can be carried out in both media in order to establish the capability of the methodology.

Also, the peculiar effects of water in some photocatalytic reactions have been uncovered, which further emphasizes the unique role of water as a solvent, particularly in photocatalytic systems, for example where, in its absence, the desired product is not formed at all, or water itself also intervenes as a proton donor or oxygen donor or is necessary in stoichiometric amounts as a coreactant. Even more intriguing effects can be observed when water plays a part in the stereoselectivity of the reaction, influencing and driving the formation of products with high diastereoselectivity as opposed to the same reactions performed in organic media. The effects of water for improving enantioselectivity in chiral catalytic photoredox reactions were also exposed and explored with representative examples.

Attention should be paid to those photocatalyzed reactions that do not work in organic solvents but do work in water or aqueous media with high product yields, as these should be considered an entry into new protocols and reaction types. Finally, there are myriads of photocatalyzed transformations in organic solvents that await to be revisited or attempted in water or aqueous media, demonstrating the potential of the methodology, the possibility of bringing to light unexpected selectivity and product yield enhancement, and ultimately diminishing the environmental impact of these organic synthetic procedures.

\section{AUTHOR INFORMATION}

\section{Corresponding Authors}

Sebastián Barata-Vallejo - Departamento de Ciencias Químicas, Facultad de Farmacia y Bioquímica, Universidad de Buenos Aires, Junín 954, CP 1113 Buenos Aires, Argentina; ISOF, Consiglio Nazionale delle Ricerche, 40129 Bologna, Italy; Email: sebastian.barata@isof.cnr.it

Al Postigo - Departamento de Ciencias Químicas, Facultad de Farmacia y Bioquímica, Universidad de Buenos Aires, Junín 954, CP 1113 Buenos Aires, Argentina; ㅇo orcid.org/00000002-4177-3689; Email: apostigo@ffyb.uba.ar

\section{Author}

Damian E. Yerien - Departamento de Ciencias Químicas, Facultad de Farmacia y Bioquímica, Universidad de Buenos Aires, Junín 954, CP 1113 Buenos Aires, Argentina

Complete contact information is available at:

https://pubs.acs.org/10.1021/acssuschemeng.1c03384

\section{Notes}

The authors declare no competing financial interest.

\section{Biographies}

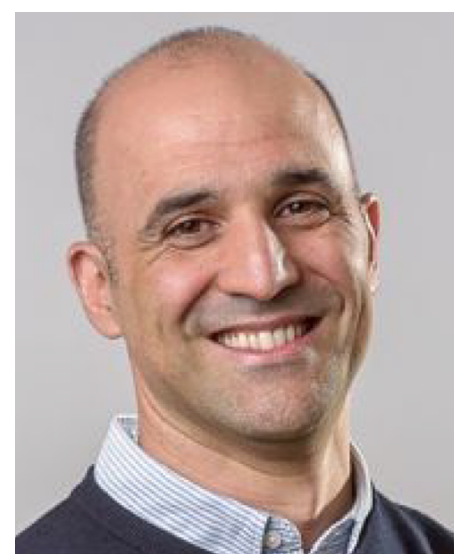

Sebastián Barata-Vallejo was born in General Villegas (Argentina) and holds degrees in pharmacy (2007) and biochemistry (2010). He obtained his Ph.D. degree (2012) at the University of Buenos Aires, studying radical reactions in aqueous and microheterogeneous media under the supervision of Prof. A. Postigo. He has been a research fellow and has held several postdoctoral positions at the Istituto per la Sintesi Organica e la Fotoreattività (ISOF), Consiglio Nazionale delle Ricerche (CNR), Bologna, Italia, under the supervision of Dr. C. Chatgilialoglu, studying biomimetic radical reactions and their mechanisms. He is currently a researcher at the National Council for Scientific and Technical Investigation, CONICET (Argentina), a research associate at ISOF-CNR, Bologna, Italy, and a lecturer in the Chemical Sciences Department, Faculty of Pharmacy and Biochemistry, University of Buenos Aires. His research activities focus on radical organic chemistry, in particular carbon- and sulfur-centered radicals reactivity, fluoroalkylation reactions by radical pathways, and photocatalysis.

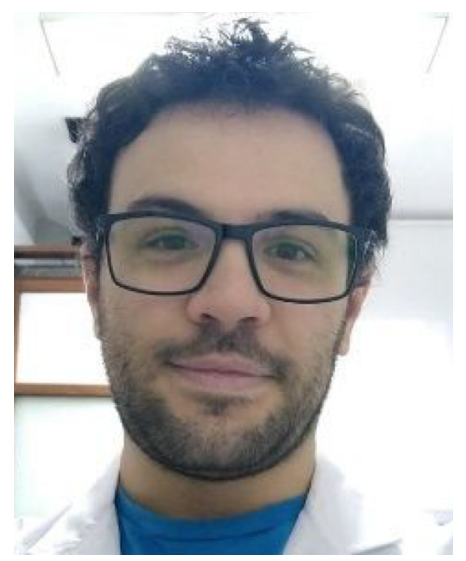

Damian E. Yerien was born in Argentina and obtained his biochemistry degree from University of Buenos Aires in 2014. He obtained his Ph.D. degree (2019) at the University of Buenos Aires studying synthetic and mechanistic aspects of radical perfluoroalkylation reactions through photoredox catalysis, under the direction of Prof. Dr. Al Postigo. He is currently a National Argentine Research Council postdoctoral scholarship holder and a teaching assistant in the Department of Chemical Sciences, Faculty of Pharmacy and Biochemistry, University of Buenos Aires. 


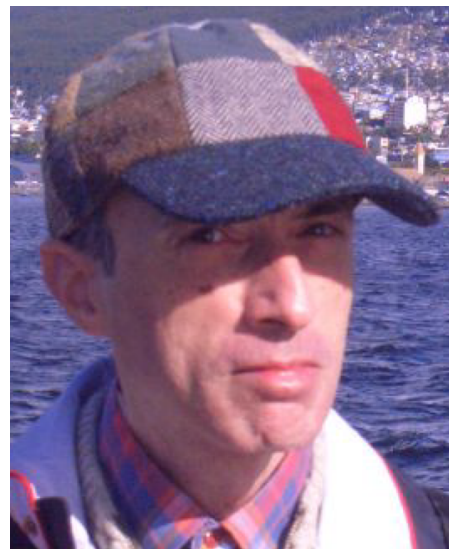

Al Postigo was born in Argentina and obtained his M.Sc. degree from the University of Buenos Aires in 1986. He moved to Canada in 1990 and obtained his Ph.D. from McMaster University in 1994, under the direction of Prof. Dr. W. J. Leigh. After postdoctoral positions in Canada, he returned to Argentina and worked with Prof. Dr. R. Rossi at the University of Córdoba in the area of radical ion reactions. He held assistant and associate professorship positions at the University of Córdoba, University of Buenos Aires, and University of Belgrano. $\mathrm{He}$ is currently full professor of organic chemistry in the Department of Chemical Sciences, Faculty of Pharmacy and Biochemistry, University of Buenos Aires. His interests are in the areas of radical chemistry, for both carbon-centered radicals and metal-centered radicals. He is devoted to studying radical reactions of these species in water and nonconventional media.

\section{ACKNOWLEDGMENTS}

D.E.Y. is thankful for a postdoctoral scholarship to Consejo Nacional de Investigaciones Científicas y Técnicas, Conicet. S.B-V. and A.P. are research members of Conicet. The following financial agencies are acknowledged: Conicet, UBASeCyT, and ANPCyT.

\section{REFERENCES}

(1) Romero, N. A.; Nicewicz, D. A. Organic Photoredox Catalysis. Chem. Rev. 2016, 116 (17), 10075-10166.

(2) Sachdeva, H. A Mini-Review on Organic Synthesis in Water. MOJ. Bioorganic Org. Chem. 2017, 1 (7), na.

(3) Chanda, A.; Fokin, V. V. Organic Synthesis "On Water. Chem. Rev. 2009, 109 (2), 725-748.

(4) Rueda-Marquez, J. J.; Levchuk, I.; Fernández Ibañez, P.; Sillanpää, M. A Critical Review on Application of Photocatalysis for Toxicity Reduction of Real Wastewaters. J. Cleaner Prod. 2020, 258, 120694.

(5) Arias-Rotondo, D. M.; McCusker, J. K. The Photophysics of Photoredox Catalysis: A Roadmap for Catalyst Design. Chem. Soc. Rev. 2016, 45 (21), 5803-5820.

(6) Sun, K.; Lv, Q.-Y.; Chen, X.-L.; Qu, L.-B.; Yu, B. Recent Advances in Visible-Light-Mediated Organic Transformations in Water. Green Chem. 2021, 23 (1), 232-248.

(7) Tobisu, M.; Koh, K.; Furukawa, T.; Chatani, N. Modular Synthesis of Phenanthridine Derivatives by Oxidative Cyclization of 2-Isocyanobiphenyls with Organoboron Reagents. Angew. Chem., Int. Ed. 2012, 51 (45), 11363-11366.

(8) Wang, Q.; Dong, X.; Xiao, T.; Zhou, L. PhI(OAc) 2 -Mediated Synthesis of 6-(Trifluoromethyl)Phenanthridines by Oxidative Cyclization of 2-Isocyanobiphenyls with CF 3 SiMe 3 under MetalFree Conditions. Org. Lett. 2013, 15 (18), 4846-4849.

(9) Liu, X.; Qing, Z.; Cheng, P.; Zheng, X.; Zeng, J.; Xie, H. MetalFree Photoredox Catalyzed Cyclization of O-(2,4-Dinitrophenyl)Oximes to Phenanthridines. Molecules 2016, 21 (12), 1690.
(10) Jiang, H.; An, X.; Tong, K.; Zheng, T.; Zhang, Y.; Yu, S. VisibleLight-Promoted Iminyl-Radical Formation from Acyl Oximes: A Unified Approach to Pyridines, Quinolines, and Phenanthridines. Angew. Chem., Int. Ed. 2015, 54 (13), 4055-4059.

(11) An, X.-D.; Yu, S. Visible-Light-Promoted and One-Pot Synthesis of Phenanthridines and Quinolines from Aldehydes and O -Acyl Hydroxylamine. Org. Lett. 2015, 17 (11), 2692-2695.

(12) Zhang, B.; Mück-Lichtenfeld, C.; Daniliuc, C. G.; Studer, A. 6Trifluoromethyl-Phenanthridines through Radical Trifluoromethylation of Isonitriles. Angew. Chem., Int. Ed. 2013, 52 (41), 1079210795 .

(13) Leifert, D.; Daniliuc, C. G.; Studer, A. 6-Aroylated Phenanthridines via Base Promoted Homolytic Aromatic Substitution (BHAS). Org. Lett. 2013, 15 (24), 6286-6289.

(14) Jiang, H.; Cheng, Y.; Wang, R.; Zheng, M.; Zhang, Y.; Yu, S. Synthesis of 6-Alkylated Phenanthridine Derivatives Using Photoredox Neutral Somophilic Isocyanide Insertion. Angew. Chem., Int. Ed. 2013, 52 (50), 13289-13292.

(15) Gu, L.; Jin, C.; Liu, J.; Ding, H.; Fan, B. Transition-Metal-Free, Visible-Light Induced Cyclization of Arylsulfonyl Chlorides with 2Isocyanobiphenyls to Produce Phenanthridines. Chem. Commun. 2014, 50 (35), 4643-4645.

(16) Xiao, T.; Li, L.; Lin, G.; Wang, Q.; Zhang, P.; Mao, Z.; Zhou, L. Synthesis of 6-Substituted Phenanthridines by Metal-Free, VisibleLight Induced Aerobic Oxidative Cyclization of 2-Isocyanobiphenyls with Hydrazines. Green Chem. 2014, 16 (5), 2418-2421.

(17) Sun, X.; Yu, S. Visible-Light-Promoted Iminyl Radical Formation from Vinyl Azides: Synthesis of 6-(Fluoro)Alkylated Phenanthridines. Chem. Commun. 2016, 52 (72), 10898-10901.

(18) Mao, L.-L.; Zheng, D.-G.; Zhu, X.-H.; Zhou, A.-X.; Yang, S.-D. Visible-Light-Induced Sulfonylation/Cyclization of Vinyl Azides: One-Pot Construction of 6-(Sulfonylmethyl)Phenanthridines. Org. Chem. Front. 2018, 5 (2), 232-236.

(19) Li, Y.; Zhu, Y.; Yang, S.-D. Visible-Light-Induced Tandem Phosphorylation Cyclization of Vinyl Azides under Mild Conditions. Org. Chem. Front. 2018, 5 (5), 822-826.

(20) Natarajan, P.; Chuskit, D.; Priya. Metal-Free, Visible-LightPromoted Oxidative Radical Cyclization of: N-Biarylglycine Esters: One-Pot Construction of Phenanthridine-6-Carboxylates in Water. Green Chem. 2019, 21 (16), 4406-4411.

(21) Zou, L.; Li, P.; Wang, B.; Wang, L. Visible-Light-Induced Radical Cyclization of N-Allylbenzamides with $\mathrm{CF} 3 \mathrm{SO} 2 \mathrm{Na}$ to Trifluoromethylated Dihydroisoquinolinones in Water at Room Temperature. Green Chem. 2019, 21 (12), 3362-3369.

(22) Huang, M. H.; Zhu, C. F.; He, C. L.; Zhu, Y. L.; Hao, W. J.; Wang, D. C.; Tu, S. J.; Jiang, B. Visible-Light-Induced Methylsulfonylation/Bicyclization of C(Sp3)-Tethered 1,7-Enynes Using a DMSO/H2O System. Org. Chem. Front. 2018, 5 (10), 1643-1650.

(23) Xia, D.; Miao, T.; Li, P.; Wang, L. Visible-Light Photoredox Catalysis: Direct Synthesis of Sulfonated Oxindoles from $N$ -Arylacrylamides and Arylsulfinic Acids by Means of a Cascade CS/C-C Formation Process. Chem. - Asian J. 2015, 10 (9), 1919-1925.

(24) Natarajan, P.; Kumar, N.; Chaudhary, R.; Venugopalan, P. Visible Light-Mediated, Rose Bengal-Catalyzed Oxidative Radical $\mathrm{C}$ [Sbnd]H Cyclization of Alkyl 1,1'-Biaryl-2-Ones: An Efficient Synthesis of 10-Alkylphenanthren-9-Ols in Water. Tetrahedron Lett. 2020, 61 (18), 151823.

(25) Vaillard, S. E.; Studer, A. Radical Arylations. In Encyclopedia of Radicals in Chemistry, Biology and Materials; John Wiley \& Sons, Ltd.: Chichester, U.K., 2012. DOI: 10.1002/9781119953678.rad032.

(26) Ghosh, I.; Marzo, L.; Das, A.; Shaikh, R; König, B. Visible Light Mediated Photoredox Catalytic Arylation Reactions. Acc. Chem. Res. 2016, 49 (8), 1566-1577.

(27) Zhang, X.; Mei, Y.; Li, Y.; Hu, J.; Huang, D.; Bi, Y. VisibleLight-Mediated Functionalization of Aryl Diazonium Salts. Asian J. Org. Chem. 2021, 10 (3), 453-463.

(28) Hari, D. P.; Schroll, P.; König, B. Metal-Free, Visible-LightMediated Direct C-H Arylation of Heteroarenes with Aryl Diazonium Salts. J. Am. Chem. Soc. 2012, 134 (6), 2958-2961. 
(29) Babu, S. S.; Muthuraja, P.; Yadav, P.; Gopinath, P. Aryldiazonium Salts in Photoredox Catalysis - Recent Trends. Adv. Synth. Catal. 2021, 363 (7), 1782-1809.

(30) Chaubey, S.; Yadav, R. K.; Kim, T. W.; Yadav, T. C.; Kumar, A.; Dwivedi, D. K.; Pandey, B. K.; Singh, A. P. Fabrication of Graphitic Carbon Nitride-Based Film: An Emerged Highly Efficient Catalyst for Direct $\mathrm{C}-\mathrm{H}$ Arylation under Solar Light. Chin. J. Chem. 2021, 39 (3), 633-639.

(31) Bu, M. J.; Lu, G. P.; Jiang, J.; Cai, C. Merging Visible-Light Photoredox and Micellar Catalysis: Arylation Reactions with Anilines Nitrosated in Situ. Catal. Sci. Technol. 2018, 8 (15), 3728-3732.

(32) Xue, D.; Jia, Z. H.; Zhao, C. J.; Zhang, Y. Y.; Wang, C.; Xiao, J. Direct Arylation of N-Heteroarenes with Aryldiazonium Salts by Photoredox Catalysis in Water. Chem. - Eur. J. 2014, 20 (10), 29602965.

(33) Tobisu, M.; Furukawa, T.; Chatani, N. Visible Light-Mediated Direct Arylation of Arenes and Heteroarenes Using Diaryliodonium Salts in the Presence and Absence of a Photocatalyst. Chem. Lett. 2013, 42 (10), 1203-1205.

(34) Wang, L.; Shen, J.; Yang, S.; Liu, W.; Chen, Q.; He, M. C-H Arylation Reactions through Aniline Activation Catalysed by a PANIg-C3N4-TiO2 Composite under Visible Light in Aqueous Medium. Green Chem. 2018, 20 (6), 1290-1296.

(35) Wang, L.; Wang, C.; Liu, W.; Chen, Q.; He, M. Visible-LightInduced Aerobic Thiocyanation of Indoles Using Reusable $\mathrm{TiO}_{2} /$ MoS2 Nanocomposite Photocatalyst. Tetrahedron Lett. 2016, 57 (16), 1771-1774.

(36) Liu, W.; Wang, C.; Huang, Y.; Chen, Q.; Wang, L.; He, M. Visible-Light-Mediated Facile Synthesis of Disulfides Using Reusable TiO2/MoS2nanocomposite Photocatalyst. Synth. Commun. 2016, 46 (15), 1268-1274.

(37) Liu, W.; Wang, C.; Wang, L. Photocatalyzed Facile Synthesis of $\alpha$-Chloro Aryl Ketones with Polyaniline-g-C3N4-TiO2 Composite under Visible Light. Ind. Eng. Chem. Res. 2017, 56 (21), 6114-6123.

(38) Liu, J.; Wu, S.; Yu, J.; Lu, C.; Wu, Z.; Wu, X.; Xue, X.; Zhu, C. Polarity Umpolung Strategy for the Radical Alkylation of Alkenes. Angew. Chem., Int. Ed. 2020, 59 (21), 8195-8202.

(39) Dong, Z.; Ren, Z.; Thompson, S. J.; Xu, Y.; Dong, G. Transition-Metal-Catalyzed C-H Alkylation Using Alkenes. Chem. Rev. 2017, 117 (13), 9333-9403.

(40) Zhou, F.; Zhu, J.; Zhang, Y.; Zhu, S. NiH-Catalyzed Reductive Relay Hydroalkylation: A Strategy for the Remote $\mathrm{C}\left(\mathrm{Sp}{ }^{3}\right)-\mathrm{H}$ Alkylation of Alkenes. Angew. Chem. 2018, 130 (15), 4122-4126.

(41) Dondi, D.; Ravelli, D.; Fagnoni, M.; Mella, M.; Molinari, A.; Maldotti, A.; Albini, A. Regio- and Stereoselectivity in the Decatungstate Photocatalyzed Alkylation of Alkenes by Alkylcyclohexanes. Chem. - Eur. J. 2009, 15 (32), 7949-7957.

(42) Gualandi, A.; Mazzarella, D.; Ortega-Martínez, A.; Mengozzi, L.; Calcinelli, F.; Matteucci, E.; Monti, F.; Armaroli, N.; Sambri, L.; Cozzi, P. G. Photocatalytic Radical Alkylation of Electrophilic Olefins by Benzylic and Alkylic Zinc-Sulfinates. ACS Catal. 2017, 7 (8), $5357-5362$.

(43) Chen, H.; Anand, D.; Zhou, L. Photoredox Defluorinative Alkylation of 1-Trifluoromethyl Alkenes and 1,3-Butadienes with 1,4Dihydropyridines as Alkylation Reagents. Asian J. Org. Chem. 2019, 8 (5), 661-664.

(44) Zhang, W.; Lin, S. Electroreductive Carbofunctionalization of Alkenes with Alkyl Bromides via a Radical-Polar Crossover Mechanism. J. Am. Chem. Soc. 2020, 142 (49), 20661-20670.

(45) Sauermann, N.; Meyer, T. H.; Qiu, Y.; Ackermann, L. Electrocatalytic C-H Activation. ACS Catal. 2018, 8 (8), 7086-7103.

(46) Yao, H.; Hu, W.; Zhang, W. Difunctionalization of Alkenes and Alkynes via Intermolecular Radical and Nucleophilic Additions. Molecules 2021, 26 (1), 105.

(47) Tzirakis, M. D.; Lykakis, I. N.; Orfanopoulos, M. Decatungstate as an Efficient Photocatalyst in Organic Chemistry. Chem. Soc. Rev. 2009, 38 (9), 2609-2621.

(48) Wen, Z.; Maheshwari, A.; Sambiagio, C.; Deng, Y.; Laudadio, G.; Van Aken, K.; Sun, Y.; Gemoets, H. P. L.; Noël, T. Optimization of a Decatungstate-Catalyzed $\mathrm{C}(\mathrm{Sp} 3)-\mathrm{H}$ Alkylation Using a Continuous Oscillatory Millistructured Photoreactor. Org. Process Res. Dev. 2020, 24 (10), 2356-2361.

(49) Ravelli, D.; Fagnoni, M.; Fukuyama, T.; Nishikawa, T.; Ryu, I. Site-Selective C-H Functionalization by Decatungstate Anion Photocatalysis: Synergistic Control by Polar and Steric Effects Expands the Reaction Scope. ACS Catal. 2018, 8, 701-713.

(50) Bonassi, F.; Ravelli, D.; Protti, S.; Fagnoni, M. Decatungstate Photocatalyzed Acylations and Alkylations in Flow via Hydrogen Atom Transfer. Adv. Synth. Catal. 2015, 357 (16-17), 3687-3695.

(51) Li, L.; Huang, M.; Liu, C.; Xiao, J. C.; Chen, Q. Y.; Guo, Y.; Zhao, Z. G. 2,2,2-Trifluoroethylation of Styrenes with Concomitant Introduction of a Hydroxyl Group from Molecular Oxygen by Photoredox Catalysis Activated by Visible Light. Org. Lett. 2015, 17 (19), 4714-4717.

(52) Lee, S.; Lim, C. J.; Kim, S.; Subramaniam, R.; Zimmerman, J.; Sibi, M. P. Enantioselective Conjugate Radical Addition to $\mathrm{A}^{\prime}$ Hydroxy Enones. Org. Lett. 2006, 8 (19), 4311-4313.

(53) Byrd, K. M. Diastereoselective and Enantioselective Conjugate Addition Reactions Utilizing $\alpha, \beta$-Unsaturated Amides and Lactams. Beilstein J. Org. Chem. 2015, 11, 530-562.

(54) Huo, H.; Harms, K.; Meggers, E. Catalytic, Enantioselective Addition of Alkyl Radicals to Alkenes via Visible-Light-Activated Photoredox Catalysis with a Chiral Rhodium Complex. J. Am. Chem. Soc. 2016, 138 (22), 6936-6939.

(55) Voutyritsa, E.; Triandafillidi, I.; Kokotos, C. G. Expanding the Scope of Photocatalysis: Atom Transfer Radical Addition of Bromoacetonitrile to Aliphatic Olefins. ChemCatChem 2018, 10 (11), 2466-2470.

(56) Wallentin, C. J.; Nguyen, J. D.; Finkbeiner, P.; Stephenson, C. R. J. Visible Light-Mediated Atom Transfer Radical Addition via Oxidative and Reductive Quenching of Photocatalysts. J. Am. Chem. Soc. 2012, 134 (21), 8875-8884.

(57) Voutyritsa, E.; Triandafillidi, I.; Tzouras, N. V.; Nikitas, N. F.; Pefkianakis, E. K.; Vougioukalakis, G. C.; Kokotos, C. G. Photocatalytic Atom Transfer Radical Addition to Olefins Utilizing Novel Photocatalysts. Molecules 2019, 24 (9), 1644.

(58) Severin, K. Ruthenium-Catalyzed Atom Transfer Radical Addition Reactions. Chimia 2012, 66 (6), 386-388.

(59) Riente, P.; Pericàs, M. A. Visible Light-Driven Atom Transfer Radical Addition to Olefins Using $\mathrm{Bi}_{2} \mathrm{O}_{3}$ as Photocatalyst. ChemSusChem 2015, 8 (11), 1841-1844.

(60) Bianchi, P.; Williams, J. D.; Kappe, C. O. Continuous Flow Processing of Bismuth-Photocatalyzed Atom Transfer Radical Addition Reactions Using an Oscillatory Flow Reactor. Green Chem. 2021, 23 (7), 2685.

(61) Tang, X.-J.; Dolbier, W. R. Efficient Cu-Catalyzed Atom Transfer Radical Addition Reactions of Fluoroalkylsulfonyl Chlorides with Electron-Deficient Alkenes Induced by Visible Light. Angew. Chem., Int. Ed. 2015, 54 (14), 4246-4249.

(62) Engl, S.; Reiser, O. Copper Makes the Difference: Visible LightMediated Atom Transfer Radical Addition Reactions of Iodoform with Olefins. ACS Catal. 2020, 10 (17), 9899-9906.

(63) Knorn, M.; Rawner, T.; Czerwieniec, R.; Reiser, O. [Copper(Phenanthroline)(Bisisonitrile)]+-Complexes for the Visible-LightMediated Atom Transfer Radical Addition and Allylation Reactions. ACS Catal. 2015, 5 (9), 5186-5193.

(64) Mao, L.-L.; Cong, H. Atom-Transfer Radical Addition to Unactivated Alkenes by Using Heterogeneous Visible-Light Photocatalysis. ChemSusChem 2017, 10 (22), 4461-4464.

(65) Zidan, M.; McCallum, T.; Swann, R.; Barriault, L. Formal Bromine Atom Transfer Radical Addition of Nonactivated Bromoalkanes Using Photoredox Gold Catalysis. Org. Lett. 2020, 22 (21), 8401-8406.

(66) Arceo, E.; Montroni, E.; Melchiorre, P. Photo-Organocatalysis of Atom-Transfer Radical Additions to Alkenes. Angew. Chem., Int. Ed. 2014, 53 (45), 12064-12068.

(67) Tiwari, D. P.; Dabral, S.; Wen, J.; Wiesenthal, J.; Terhorst, S.; Bolm, C. Organic Dye-Catalyzed Atom Transfer Radical Addition- 
Elimination (ATRE) Reaction for the Synthesis of Perfluoroalkylated Alkenes. Org. Lett. 2017, 19 (16), 4295-4298.

(68) Matsuo, K.; Yamaguchi, E.; Itoh, A. In Situ-Generated Halogen-Bonding Complex Enables Atom Transfer Radical Addition (ATRA) Reactions of Olefins. J. Org. Chem. 2020, 85 (16), 1057410583.

(69) Xu, W.-W.; Wang, L.; Mao, T.; Gu, J.; Li, X.-F.; He, C.-Y. Visible-Light Promoted Atom Transfer Radical Addition-Elimination (ATRE) Reaction for the Synthesis of Fluoroalkylated Alkenes Using DMA as Electron-Donor. Molecules 2020, 25 (3), 508.

(70) Zhang, T.; Wang, P.; Gao, Z.; An, Y.; He, C.; Duan, C. PyreneBased Metal-Organic Framework NU-1000 Photocatalysed AtomTransfer Radical Addition for Iodoperfluoroalkylation and (Z)Selective Perfluoroalkylation of Olefins by Visible-Light Irradiation. RSC Adv. 2018, 8 (57), 32610-32620.

(71) Muñoz-Molina, J. M.; Caballero, A.; Díaz-Requejo, M. M.; Trofimenko, S.; Belderraín, T. R.; Pérez, P. J. Copper-Homoscorpionate Complexes as Active Catalysts for Atom Transfer Radical Addition to Olefins. Inorg. Chem. 2007, 46 (19), 7725-7730.

(72) Pu, W.; Sun, D.; Fan, W.; Pan, W.; Chai, Q.; Wang, X.; Lv, Y. $\mathrm{Cu}$-Catalyzed Atom Transfer Radical Addition Reactions of Alkenes with $\alpha$-Bromoacetonitrile. Chem. Commun. 2019, 55 (33), 48214824.

(73) Nguyen, J. D.; Tucker, J. W.; Konieczynska, M. D.; Stephenson, C. R. J. Intermolecular Atom Transfer Radical Addition to Olefins Mediated by Oxidative Quenching of Photoredox Catalysts. J. Am. Chem. Soc. 2011, 133 (12), 4160-4163.

(74) Slodowicz, M.; Barata-Vallejo, S.; Vázquez, A.; Nudelman, N. S.; Postigo, A. Light-Induced Iodoperfluoroalkylation Reactions of Carbon-Carbon Multiple Bonds in Water. J. Fluorine Chem. 2012, 135, 137-143.

(75) Yajima, T.; Ikegami, M. Metal-Free Visible-Light Radical Iodoperfluoroalkylation of Terminal Alkenes and Alkynes. Eur. J. Org. Chem. 2017, 2017 (15), 2126-2129.

(76) Yerien, D. E.; Barata-Vallejo, S.; Mora Flores, E. W.; Postigo, A. The Role of Photocatalysts in Radical Chains in Homolytic Aromatic Substitution, Radical Addition to Olefins, and Nucleophilic Radical Substitution Mechanisms. Catal. Sci. Technol. 2020, 10 (15), 51135128.

(77) Rosso, C.; Filippini, G.; Cozzi, P. G.; Gualandi, A.; Prato, M. Highly Performing Iodoperfluoroalkylation of Alkenes Triggered by the Photochemical Activity of Perylene Diimides. ChemPhotoChem. 2019, 3 (4), 193-197.

(78) Renaud, P.; Vionnet, J. P.; Vogel, P. Radical Additions to Silyl Ketene Acetals. Introduction of C-Moieties to 7-Oxabicyclo[2.2.1]Hept-5-En-2-Endo-Ol. Tetrahedron Lett. 1991, 32 (29), 3491-3494.

(79) Norman, A. R.; Yousif, M. N.; McErlean, C. S. P. PhotoredoxCatalyzed Indirect Acyl Radical Generation from Thioesters. Org. Chem. Front. 2018, 5 (22), 3267-3298.

(80) Banerjee, A.; Lei, Z.; Ngai, M. Y. Acyl Radical Chemistry via Visible-Light Photoredox Catalysis. Synthesis 2019, 51 (2), 303-333.

(81) Zhang, X.; Qin, J.; Huang, X.; Meggers, E. One-Pot Sequential Photoredox Chemistry and Asymmetric Transfer Hydrogenation with a Single Catalyst. Eur. J. Org. Chem. 2018, 2018 (4), 571-577.

(82) Jana, K.; Bhunia, A.; Studer, A. Radical 1,3-Difunctionalization of Allylboronic Esters with Concomitant 1,2-Boron Shift. Chem. 2020, 6 (2), 512-522.

(83) Zhu, X.; Deng, W.; Chiou, M. F.; Ye, C.; Jian, W.; Zeng, Y.; Jiao, Y.; Ge, L.; Li, Y.; Zhang, X.; et al. Copper-Catalyzed Radical 1,4Difunctionalization of 1,3-Enynes with Alkyl Diacyl Peroxides and NFluorobenzenesulfonimide. J. Am. Chem. Soc. 2019, 141 (1), 548559.

(84) Liu, J.; Yao, H.; Li, X.; Wu, H.; Lin, A.; Yao, H.; Xu, J.; Xu, S. Organocatalytic 1,5-Trifluoromethylthio-Sulfonylation of Vinylcyclopropane Mediated by Visible Light in the Water Phase. Org. Chem. Front. 2020, 7 (11), 1314-1320.

(85) Wang, Q. L.; Zhang, W. Z.; Zhou, Q.; Zhou, C. S.; Xiong, B. Q.; Tang, K. W.; Liu, Y. Visible-Light-Mediated Difunctionalization of
Vinylcyclopropanes for the Synthesis of 1-Sulfonylmethyl-3,4Dihydronaphthalenes. Org. Biomol. Chem. 2019, 17 (34), 7918-7926.

(86) Ackermann, L.; Born, R.; Álvarez-Bercedo, P. Ruthenium(IV) Alkylidenes as Precatalysts for Direct Arylations of Alkenes with Aryl Chlorides and an Application to Sequential Catalysis. Angew. Chem., Int. Ed. 2007, 46 (33), 6364-6367.

(87) Hari, D. P.; König, B. The Photocatalyzed Meerwein Arylation: Classic Reaction of Aryl Diazonium Salts in a New Light. Angew. Chem., Int. Ed. 2013, 52, 4734-4743.

(88) Shi, L.; Wang, Y.; Yang, H.; Fu, H. Copper-Catalyzed BisArylations of Alkenes Leading to Oxindole Derivatives. Org. Biomol. Chem. 2014, 12 (24), 4070-4073.

(89) Prasad Hari, D.; Hering, T.; König, B. The PhotoredoxCatalyzed Meerwein Addition Reaction: Intermolecular AminoArylation of Alkenes. Angew. Chem., Int. Ed. 2014, 53 (3), 725-728.

(90) Wang, J.; Xue, L.; Hong, M.; Ni, B.; Niu, T. Heterogeneous Visible-Light-Induced Meerwein Hydration Reaction of Alkenes in Water Using Mpg-C3N4 as a Recyclable Photocatalyst. Green Chem. 2020, 22 (2), 411-416.

(91) Bloom, S.; Chilamari, M.; Immel, J. R. General Access to CCentered Radicals: Combining a Bioinspired Photocatalyst with Boronic Acids in Aqueous Media. ACS Catal. 2020, 10 (21), 1272712737.

(92) Goti, G.; Bieszczad, B.; Vega-Peñaloza, A.; Melchiorre, P. Stereocontrolled Synthesis of 1,4-Dicarbonyl Compounds by Photochemical Organocatalytic Acyl Radical Addition to Enals. Angew. Chem., Int. Ed. 2019, 58 (4), 1213-1217.

(93) Zhao, J. J.; Zhang, H. H.; Shen, X.; Yu, S. Enantioselective Radical Hydroacylation of Enals with $\alpha$-Ketoacids Enabled by Photoredox/Amine Cocatalysis. Org. Lett. 2019, 21 (4), 913-916.

(94) Kuang, Y.; Wang, K.; Shi, X.; Huang, X.; Meggers, E.; Wu, J. Asymmetric Synthesis of 1,4-Dicarbonyl Compounds from Aldehydes by Hydrogen Atom Transfer Photocatalysis and Chiral Lewis Acid Catalysis. Angew. Chem., Int. Ed. 2019, 58 (47), 16859-16863.

(95) Hari, D. P.; König, B. Synthetic Applications of Eosin Y in Photoredox Catalysis. Chem. Commun. 2014, 50 (51), 6688-6699.

(96) Zuo, Z.; Cong, H.; Li, W.; Choi, J.; Fu, G. C.; MacMillan, D. W. C. Enantioselective Decarboxylative Arylation of $\alpha$-Amino Acids via the Merger of Photoredox and Nickel Catalysis. J. Am. Chem. Soc. 2016, 138 (6), 1832-1835.

(97) Johnston, C. P.; Smith, R. T.; Allmendinger, S.; MacMillan, D. W. C. Metallaphotoredox-Catalysed Sp3-Sp3 Cross-Coupling of Carboxylic Acids with Alkyl Halides. Nature 2016, 536 (7616), $322-325$.

(98) Song, H. T.; Ding, W.; Zhou, Q. Q.; Liu, J.; Lu, L. Q.; Xiao, W. J. Photocatalytic Decarboxylative Hydroxylation of Carboxylic Acids Driven by Visible Light and Using Molecular Oxygen. J. Org. Chem. 2016, 81 (16), 7250-7255.

(99) Schwarz, J.; König, B. Decarboxylative Reactions with and without Light - a Comparison. Green Chem. 2018, 20 (2), 323-361.

(100) Chu, L.; Ohta, C.; Zuo, Z.; MacMillan, D. W. C. Carboxylic Acids as A Traceless Activation Group for Conjugate Additions: A Three-Step Synthesis of ( \pm )-Pregabalin. J. Am. Chem. Soc. 2014, 136 (31), 10886-10889.

(101) Cao, H.; Jiang, H.; Feng, H.; Kwan, J. M. C.; Liu, X.; Wu, J. Photo-Induced Decarboxylative Heck-Type Coupling of Unactivated Aliphatic Acids and Terminal Alkenes in the Absence of Sacrificial Hydrogen Acceptors. J. Am. Chem. Soc. 2018, 140 (47), 1636016367.

(102) Ventre, S.; Petronijevic, F. R.; Macmillan, D. W. C. Decarboxylative Fluorination of Aliphatic Carboxylic Acids via Photoredox Catalysis. J. Am. Chem. Soc. 2015, 137 (17), 5654-5657.

(103) Wu, X.; Meng, C.; Yuan, X.; Jia, X.; Qian, X.; Ye, J. TransitionMetal-Free Visible-Light Photoredox Catalysis at Room-Temperature for Decarboxylative Fluorination of Aliphatic Carboxylic Acids by Organic Dyes. Chem. Commun. 2015, 51 (59), 11864-11867.

(104) Li, C.; Wang, J.; Barton, L. M.; Yu, S.; Tian, M.; Peters, D. S.; Kumar, M.; Yu, A. W.; Johnson, K. A.; Chatterjee, A. K.; et al. 
Decarboxylative Borylation. Science (Washington, DC, U. S.) 2017, 356 (6342), No. eaam7355.

(105) Fawcett, A.; Pradeilles, J.; Wang, Y.; Mutsuga, T.; Myers, E. L.; Aggarwal, V. K. Photoinduced Decarboxylative Borylation of Carboxylic Acids. Science (Washington, DC, U. S.) 2017, 357 (6348), 283-286.

(106) Hu, X. Q.; Liu, Z. K.; Hou, Y. X.; Gao, Y. Single Electron Activation of Aryl Carboxylic Acids. iScience 2020, 23, 101266.

(107) Zeng, Z.; Feceu, A.; Sivendran, N.; Goóßen, L. J. Decarboxylation-Initiated Intermolecular Carbon-Heteroatom Bond Formation. Adv. Synth. Catal. 2021, 363, 2678-2722.

(108) Xuan, J.; Zhang, Z. G.; Xiao, W. J. Visible-Light-Induced Decarboxylative Functionalization of Carboxylic Acids and Their Derivatives. Angew. Chem., Int. Ed. 2015, 54, 15632-15641.

(109) Hu, X. Q.; Hou, Y. X.; Liu, Z. K.; Gao, Y. Recent Advances in Phosphoranyl Radical-Mediated Deoxygenative Functionalisation. Org. Chem. Front. 2020, 7 (16), 2319-2324.

(110) Leca, D.; Fensterbank, L.; Lacôte, E.; Malacria, M. Recent Advances in the Use of Phosphorus-Centered Radicals in Organic Chemistry. Chem. Soc. Rev. 2005, 34 (10), 858-865.

(111) Rossi-Ashton, J. A.; Clarke, A. K.; Unsworth, W. P.; Taylor, R. J. K. Phosphoranyl Radical Fragmentation Reactions Driven by Photoredox Catalysis. ACS Catal. 2020, 10, 7250-7261.

(112) Xu, J.; Arkin, M.; Peng, Y.; Xu, W.; Yu, H.; Lin, X.; Wu, Q. Enantiocomplementary Decarboxylative Hydroxylation Combining Photocatalysis and Whole-Cell Biocatalysis in a One-Pot Cascade Process. Green Chem. 2019, 21 (8), 1907-1911.

(113) Ni, Y.; Li, C. X.; Ma, H. M.; Zhang, J.; Xu, J. H. Biocatalytic Properties of a Recombinant Aldo-Keto Reductase with Broad Substrate Spectrum and Excellent Stereoselectivity. Appl. Microbiol. Biotechnol. 2011, 89 (4), 1111-1118.

(114) Ni, Y.; Li, C. X.; Wang, L. J.; Zhang, J.; Xu, J. H. Highly Stereoselective Reduction of Prochiral Ketones by a Bacterial Reductase Coupled with Cofactor Regeneration. Org. Biomol. Chem. 2011, 9 (15), 5463-5468.

(115) Tang, C.; Qiu, X.; Cheng, Z.; Jiao, N. Molecular OxygenMediated Oxygenation Reactions Involving Radicals. Chem. Soc. Rev. 2021, 50, 8067-8101, DOI: 10.1039/D1CS00242B.

(116) Zhang, M.; Xie, J.; Zhu, C. A General Deoxygenation Approach for Synthesis of Ketones from Aromatic Carboxylic Acids and Alkenes. Nat. Commun. 2018, 9 (1), 1-10.

(117) Xie, L. Y.; Fang, T. G.; Tan, J. X.; Zhang, B.; Cao, Z.; Yang, L. H.; He, W. M. Visible-Light-Induced Deoxygenative C2-Sulfonylation of Quinoline: N -Oxides with Sulfinic Acids. Green Chem. 2019, 21 (14), 3858-3863.

(118) Rueda-Becerril, M.; Mahé, O.; Drouin, M.; Majewski, M. B.; West, J. G.; Wolf, M. O.; Sammis, G. M.; Paquin, J. F. Direct C-F Bond Formation Using Photoredox Catalysis. J. Am. Chem. Soc. 2014, 136 (6), 2637-2641.

(119) Wallin, S.; Davidsson, J.; Modin, J.; Hammarström, L. Femtosecond Transient Absorption Anisotropy Study on $[\mathrm{Ru}(\mathrm{Bpy})$ $3] 2+$ and $[\mathrm{Ru}(\mathrm{Bpy})(\mathrm{Py}) 4] 2+\cdot$ Ultrafast Interligand Randomization of the MLCT State. J. Phys. Chem. A 2005, 109 (21), 4697-4704.

(120) Chen, H.; He, Y.; Zhou, L. A Photocatalytic Decarboxylative/ Defluorinative [4 + 3] Annulation of o-Hydroxyphenylacetic Acids and Trifluoromethyl Alkenes: Synthesis of Fluorinated Dihydrobenzoxepines. Org. Chem. Front. 2018, 5 (22), 3240-3244.

(121) Zhang, M.; Xi, J.; Ruzi, R.; Li, N.; Wu, Z.; Li, W.; Zhu, C. Domino-Fluorination-Protodefluorination Enables Decarboxylative Cross-Coupling of $\alpha$-Oxocarboxylic Acids with Styrene via Photoredox Catalysis. J. Org. Chem. 2017, 82 (18), 9305-9311.

(122) Ramirez, N. P.; Gonzalez-Gomez, J. C. Decarboxylative GieseType Reaction of Carboxylic Acids Promoted by Visible Light: A Sustainable and Photoredox-Neutral Protocol. Eur. J. Org. Chem. 2017, 2017 (15), 2154-2163.

(123) Wang, G. Z.; Shang, R.; Cheng, W. M.; Fu, Y. Decarboxylative 1,4-Addition of $\alpha$-Oxocarboxylic Acids with Michael Acceptors Enabled by Photoredox Catalysis. Org. Lett. 2015, 17 (19), 48304833.
(124) Chen, C.; Wang, X.; Yang, T. Recent Synthetic Applications of the Hypervalent Iodine(III) Reagents in Visible-Light-Induced Photoredox Catalysis. Front. Chem. 2020, 8, 551159.

(125) Huang, H.; Jia, K.; Chen, Y. Hypervalent Iodine Reagents Enable Chemoselective Deboronative/Decarboxylative Alkenylation by Photoredox Catalysis. Angew. Chem. 2015, 127 (6), 1901-1904.

(126) Zhang, J. J.; Cheng, Y. B.; Duan, X. H. Metal-Free Oxidative Decarboxylative Acylation/Ring Expansion of Vinylcyclobutanols with $\alpha$-Keto Acids by Visible Light Photoredox Catalysis. Chin. J. Chem. 2017, 35 (3), 311-315.

(127) Zilate, B.; Fischer, C.; Sparr, C. Design and Application of Aminoacridinium Organophotoredox Catalysts. Chem. Commun. 2020, 56 (12), 1767-1775.

(128) Savateev, A.; Ghosh, I.; König, B.; Antonietti, M. Photoredox Catalytic Organic Transformations Using Heterogeneous Carbon Nitrides. Angew. Chem., Int. Ed. 2018, 57 (49), 15936-15947.

(129) Barata-Vallejo, S.; Postigo, A. New Visible-Light-Triggered Photocatalytic Trifluoromethylation Reactions of Carbon-Carbon Multiple Bonds and (Hetero)Aromatic Compounds. Chem. - Eur. J. 2020, 26 (49), 11065-11084.

(130) Cai, Y.; Tang, Y.; Fan, L.; Lefebvre, Q.; Hou, H.; Rueping, M. Heterogeneous Visible-Light Photoredox Catalysis with Graphitic Carbon Nitride for $\alpha$-Aminoalkyl Radical Additions, Allylations, and Heteroarylations. ACS Catal. 2018, 8 (10), 9471-9476.

(131) Wang, X.; Maeda, K.; Thomas, A.; Takanabe, K.; Xin, G.; Carlsson, J. M.; Domen, K.; Antonietti, M. A Metal-Free Polymeric Photocatalyst for Hydrogen Production from Water under Visible Light. Nat. Mater. 2009, 8 (1), 76-80.

(132) Groenewolt, M.; Antonietti, M. Synthesis of G-C3N4 Nanoparticles in Mesoporous Silica Host Matrices. Adv. Mater. 2005, 17 (14), 1789-1792.

(133) Lakhi, K. S.; Park, D.-H.; Al-Bahily, K.; Cha, W.; Viswanathan, B.; Choy, J.-H.; Vinu, A. Mesoporous Carbon Nitrides: Synthesis, Functionalization, and Applications. Chem. Soc. Rev. 2017, 46 (1), $72-101$.

(134) Ghosh, I.; Khamrai, J.; Savateev, A.; Shlapakov, N.; Antonietti, M.; König, B. Organic Semiconductor Photocatalyst Can Bifunctionalize Arenes and Heteroarenes. Science (Washington, DC, U. S.) 2019, 365 (6451), 360-366.

(135) Geng, P.; Tang, Y.; Pan, G.; Wang, W.; Hu, J.; Cai, Y. A GC3N4-Based Heterogeneous Photocatalyst for Visible Light Mediated Aerobic Benzylic C-H Oxygenations. Green Chem. 2019, 21 (22), 6116-6122.

(136) Zhao, L.-M.; Meng, Q.-Y.; Fan, X.-B.; Ye, C.; Li, X.-B.; Chen, B.; Ramamurthy, V.; Tung, C.-H.; Wu, L.-Z. Photocatalysis with Quantum Dots and Visible Light: Selective and Efficient Oxidation of Alcohols to Carbonyl Compounds through a Radical Relay Process in Water. Angew. Chem., Int. Ed. 2017, 56 (11), 3020-3024.

(137) Pandey, G.; Kumaraswamy, G. A Novel and Selective Procedure for Generating Iminium Cation by Photosensitized (Set) Oxidative Reaction. Tetrahedron Lett. 1988, 29 (33), 4153-4156.

(138) Pandey, G.; Kumaraswamy, G.; Reddy, P. Y. Regioselective Generation of Iminium Cation by PET Processes: Its in Situ Trapping by Intramolecular Nucleophiles and Synthesis of Some Biologically Active Heterocycles. Tetrahedron 1992, 48 (38), 8295-8308.

(139) Pandey, G.; Rani, K. S.; Lakshmaiah, G. Direct CarbonCarbon Bond Formation Strategy at $\alpha$-Position of Tertiary Amines by Photoinduced Electron Transfer (PET) Processes. Tetrahedron Lett. 1992, 33 (35), 5107-5110.

(140) Hou, H.; Zhu, S.; Pan, F.; Rueping, M. Visible-Light Photoredox-Catalyzed Synthesis of Nitrones: Unexpected Rate Acceleration by Water in the Synthesis of Isoxazolidines. Org. Lett. 2014, 16 (11), 2872-2875.

(141) Rajasekar Reddy, A.; Guo, Z.; Siu, F. M.; Lok, C. N.; Liu, F.; Yeung, K. C.; Zhou, C. Y.; Che, C. M. Diastereoselective Ruthenium Porphyrin-Catalyzed Tandem Nitrone Formation/1,3-Dipolar Cycloaddition for Isoxazolidines. Synthesis, in Silico Docking Study and in Vitro Biological Activities. Org. Biomol. Chem. 2012, 10 (46), 91659174. 
(142) Xu, Z. J.; Zhu, D.; Zeng, X.; Wang, F.; Tan, B.; Hou, Y.; Lv, Y.; Zhong, G. Diastereoselective HOTf-Catalyzed Three-Component One-Pot 1,3-Dipolar Cycloaddition of $\alpha$-Diazo Ester, Nitrosobenzene and Electron-Deficient Alkene. Chem. Commun. 2010, 46 (14), 2504-2506.

(143) Jana, S.; Verma, A.; Kadu, R.; Kumar, S. Visible-Light-Induced Oxidant and Metal-Free Dehydrogenative Cascade Trifluoromethylation and Oxidation of 1,6-Enynes with Water. Chem. Sci. 2017, 8 (9), 6633-6644.

(144) Lehmann, M. W.; Evans, D. H. Anomalous Behavior in the Two-Step Reduction of Quinones in Acetonitrile. J. Electroanal. Chem. 2001, 500 (1-2), 12-20.

(145) Hossain, A.; Pagire, S. K.; Reiser, O. Visible-Light-Mediated Synthesis of Pyrazines from Vinyl Azides Utilizing a Photocascade Process. Synlett 2017, 28 (14), 1707-1714.

(146) Hu, D.; Wang, L.; Li, P. Decarboxylative Borylation of Aliphatic Esters under Visible-Light Photoredox Conditions. Org. Lett. 2017, 19 (10), 2770-2773.

(147) Lei, T.; Wei, S.; Feng, K.; Chen, B.; Tung, C.; Wu, L. Borylation of Diazonium Salts by Highly Emissive and Crystalline Carbon Dots in Water. ChemSusChem 2020, 13 (7), 1715-1719.

(148) Cai, B. G.; Xuan, J.; Xiao, W. J. Visible Light-Mediated C-]P Bond Formation Reactions. Science Bulletin 2019, 64, 337-350.

(149) Rueping, M.; Zhu, S.; Koenigs, R. M. Photoredox Catalyzed C-P Bond Forming Reactions - Visible Light Mediated Oxidative Phosphonylations of Amines. Chem. Commun. 2011, 47 (30), 86798681

(150) Kafarski, P.; Lejczak, B. Biological Activity of Aminophosphonic Acids. Phosphorus, Sulfur Silicon Relat. Elem. 1991, 63 (1-2), 193-215.

(151) Wang, H.; Li, Y.; Tang, Z.; Wang, S.; Zhang, H.; Cong, H.; Lei, A. Z-Selective Addition of Diaryl Phosphine Oxides to Alkynes via Photoredox Catalysis. ACS Catal. 2018, 8 (11), 10599-10605.

(152) Liu, X.; Chen, X.; Liu, Y.; Sun, K.; Peng, Y.; Qu, L.; Yu, B. Visible-Light-Induced Metal-Free Synthesis of 2-Phosphorylated Thioflavones in Water. ChemSusChem 2020, 13 (2), 298-303.

(153) Yuan, X. Y.; Zeng, F. L.; Zhu, H. L.; Liu, Y.; Lv, Q. Y.; Chen, X. L.; Peng, L.; Yu, B. A Metal-Free Visible-Light-Promoted Phosphorylation/Cyclization Reaction in Water towards 3-Phosphorylated Benzothiophenes. Org. Chem. Front. 2020, 7 (14), 18841889.

(154) Maidan, R.; Goren, Z.; Willner, I.; Becker, J. Y. Application of Multielectron Charge Relays in Chemical and Photochemical Debromination Processes. The Role of Induced Disproportionation of N,N'-Dioctyl-4,4'-Bipyridinium Radical Cation in Two-Phase Systems. J. Am. Chem. Soc. 1984, 106 (21), 6217-6222.

(155) Goren, Z.; Willner, I. Photochemical and Chemical Reduction of Vicinal Dibromides via Phase Transfer of 4,4'-Bipyridinium Radical: The Role of Radical Disproportionation. J. Am. Chem. Soc. 1983, 105 (26), 7764-7765.

(156) Zou, Y. Q.; Guo, W.; Liu, F. L.; Lu, L. Q.; Chen, J. R.; Xiao, W. J. Visible-Light-Induced Photocatalytic Formyloxylation Reactions of 3-Bromooxindoles with Water and DMF: The Scope and Mechanism. Green Chem. 2014, 16 (8), 3787-3795.

(157) Gualandi, A.; Rodeghiero, G.; Faraone, A.; Patuzzo, F.; Marchini, M.; Calogero, F.; Perciaccante, R.; Jansen, T. P.; Ceroni, P.; Cozzi, P. G. Allylation of Aldehydes by Dual Photoredox and Nickel Catalysis. Chem. Commun. 2019, 55 (48), 6838-6841.

(158) Li, Y. L.; Li, W. D.; Gu, Z. Y.; Chen, J.; Xia, J. B. Photoredox Ni-Catalyzed Branch-Selective Reductive Coupling of Aldehydes with 1,3-Dienes. ACS Catal. 2020, 10 (2), 1528-1534.

(159) Schwarz, J. L.; Schäfers, F.; Tlahuext-Aca, A.; Lückemeier, L.; Glorius, F. Diastereoselective Allylation of Aldehydes by Dual Photoredox and Chromium Catalysis. J. Am. Chem. Soc. 2018, 140 (40), 12705-12709.

(160) Mitsunuma, H.; Tanabe, S.; Fuse, H.; Ohkubo, K.; Kanai, M. Catalytic Asymmetric Allylation of Aldehydes with Alkenes through Allylic C(Sp3)-H Functionalization Mediated by Organophotoredox and Chiral Chromium Hybrid Catalysis. Chem. Sci. 2019, 10 (12), 3459-3465.

(161) Schwarz, J. L.; Huang, H. M.; Paulisch, T. O.; Glorius, F. Dialkylation of 1,3-Dienes by Dual Photoredox and Chromium Catalysis. ACS Catal. 2020, 10 (2), 1621-1627.

(162) Gualandi, A.; Calogero, F.; Mazzarini, M.; Guazzi, S.; Fermi, A.; Bergamini, G.; Cozzi, P. G. Cp2TiCl2-Catalyzed Photoredox Allylation of Aldehydes with Visible Light. ACS Catal. 2020, 10 (6), 3857-3863.

(163) Fermi, A.; Gualandi, A.; Bergamini, G.; Cozzi, P. G. Shining Light on $\mathrm{Ti}^{\text {IV }}$ Complexes: Exceptional Tools for Metallaphotoredox Catalysis. Eur. J. Org. Chem. 2020, 2020 (45), 6955-6965.

(164) Gualandi, A.; Rodeghiero, G.; Perciaccante, R.; Jansen, T. P.; Moreno-Cabrerizo, C.; Foucher, C.; Marchini, M.; Ceroni, P.; Cozzi, P. G. Catalytic Photoredox Allylation of Aldehydes Promoted by a Cobalt Complex. Adv. Synth. Catal. 2021, 363 (4), 1105-1111.

(165) Wada, M.; Akiba, K. ya. Metallic Bismuth Mediated Allylation of Aldehydes to Homoallylic Alcohols. Tetrahedron Lett. 1985, 26 (35), 4211-4212.

(166) Wada, M.; Ohki, H.; Akiba, K. A Grignard-Type Addition of Allyl Unit to Aldehydes by Using Bismuth and Bismuth Salt. Bull. Chem. Soc. Jpn. 1990, 63 (6), 1738-1747.

(167) Miyoshi, N.; Nishio, M.; Murakami, S.; Fukuma, T.; Wada, M. A Convenient One Pot Allylation of Aldehydes with Allylic Halides Prepared in Situ from Allylic Alcohols in the Presence of Metallic Bismuth. Bull. Chem. Soc. Jpn. 2000, 73 (3), 689-692.

(168) Andrews, P. C.; Peatt, A. C.; Raston, C. L. Metal Mediated Solvent Free Synthesis of Homoallylic Alcohols. Green Chem. 2001, 3 (6), 313-315.

(169) Miyamoto, H.; Daikawa, N.; Tanaka, K. Carbon-Carbon Bond Formation Using Bismuth in a Water Medium. Tetrahedron Lett. 2003, 44 (36), 6963-6964.

(170) Smith, K.; Lock, S.; El-Hiti, G. A.; Wada, M.; Miyoshi, N. A Convenient Procedure for Bismuth-Mediated Barbier-Type Allylation of Aldehydes in Water Containing Fluoride Ions. Org. Biomol. Chem. 2004, 2 (6), 935-938.

(171) Dam, J. H.; Fristrup, P.; Madsen, R. Combined Experimental and Theoretical Mechanistic Investigation of the Barbier Allylation in Aqueous Media. J. Org. Chem. 2008, 73 (8), 3228-3235.

(172) Potenti, S.; Gualandi, A.; Puggioli, A.; Fermi, A.; Bergamini, G.; Cozzi, P. G. Photoredox Allylation Reactions Mediated by Bismuth in Aqueous Conditions. Eur. J. Org. Chem. 2021, 2021 (11), 1624-1627.

(173) Speckmeier, E.; Fischer, T. G.; Zeitler, K. A Toolbox Approach to Construct Broadly Applicable Metal-Free Catalysts for Photoredox Chemistry: Deliberate Tuning of Redox Potentials and Importance of Halogens in Donor-Acceptor Cyanoarenes. J. Am. Chem. Soc. 2018, 140 (45), 15353-15365. 